EVALUATING THE EFFECTS OF MINDFULNESS-BASED STRESS REDUCTION ON COGNITIVE FUNCTION AND WELL-BEING IN CAREGIVERS OF PERSONS WITH DEMENTIA AND RELATED NEURODEGENERATIVE DISEASES

$$
\text { by }
$$

Sasha Mallya

Master of Arts, Ryerson University, Toronto, Ontario, 2014

Bachelor of Arts (Honours), Ryerson University, Toronto, Ontario, 2010

\author{
A dissertation \\ presented to Ryerson University \\ in partial fulfillment of the \\ requirements for the degree of \\ Doctor of Philosophy \\ in the program of \\ Clinical Psychology \\ Toronto, Ontario, Canada, 2018 \\ (C) Sasha Mallya, 2018
}




\section{Author's Declaration for Electronic Submission of a Dissertation}

I hereby declare that I am the sole author of this dissertation. This is a true copy of the dissertation, including any required final revisions, as accepted by my examiners.

I authorize Ryerson University to lend this dissertation to other institutions or individuals for the purpose of scholarly research.

I further authorize Ryerson University to reproduce this dissertation by photocopying or by other means, in total or in part, at the request of other institutions or individuals for the purpose of scholarly research.

I understand that my dissertation may be made electronically available to the public. 


\title{
Evaluating the Effects of Mindfulness-Based Stress Reduction on Cognitive Function and Well-being in Caregivers of Persons with Dementia and Related Neurodegenerative
}

Diseases

\section{Doctor of Philosophy, 2018}

\author{
Sasha Mallya
}

Clinical Psychology

Ryerson University

\begin{abstract}
Approximately $14.9 \%$ of Canadians aged 65 and older are living with cognitive impairment, including dementia, which means that there is likely an equal or even greater number of Canadians involved in their care. Relative to non-caregivers, caregivers of persons with dementia typically show more cognitive difficulties (e.g., executive function, memory), and report increased perceived stress, depression, and poor quality of life. The first aim of this dissertation was to compare caregivers' cognitive and psychological function with that of non-caregiving older adults. The second aim was to assess whether a standardized mindfulness-based stress reduction (MBSR) intervention could improve cognitive function and well-being in caregivers, compared to a psychoeducational control group. In Study 1, 57 older adult caregivers completed an assessment of cognitive and psychosocial functioning. Their performance was compared with that of 97 healthy, non-caregiving older adults who completed a similar battery of measures. It was hypothesized that caregivers would show decreased performance on measures of cognitive function, as well as significantly poorer psychosocial outcomes relative to the non-caregiver group. In Study 2, caregivers were randomized into an eight week MBSR program $(n=33)$ or a psychoeducation control group $(n=24)$. At post-program, all participants completed a re-
\end{abstract}


assessment of cognitive and psychosocial functioning. Results of Study 1 showed that relative to non-caregivers, caregivers made significantly more errors on a measure of cognitive flexibility, generated fewer words on measures of phonemic fluency and semantic fluency, and learned significantly fewer words on a list learning task. Caregivers also reported significantly more stress and depressive symptoms, and reported lower quality of life. Results of Study 2 showed that post-intervention cognitive performance did not significantly differ as a function of group assignment. However, participants in the MBSR group showed a significantly larger reduction in depression and perceived stress relative to controls. Results of this RCT provide preliminary evidence for the selective benefits of MBSR relative to psychoeducation in a group of older caregivers of individuals with dementia. 


\section{Acknowledgements}

This dissertation was funded by a research grant from the Mind \& Life Institute. I have personally been funded by the Province of Ontario (Ontario Graduate Scholarship) and the Ontario Mental Health Foundation. I am extremely grateful for the support shown by the scientific community to pursue this research.

I don't think I could ever adequately thank my supervisor, Dr. Alexandra Fiocco, for the potential she saw in me, for sharing her wealth of information on stress and aging research with me, and for cultivating my thirst for knowledge and development. I will be forever grateful for the opportunities you made available to me.

I would also like to acknowledge Dr. Karen Milligan, who in addition to serving as a member of my committee, is also an incredibly supportive, kind, patient, and thoughtful mentor. I am also indebted to Drs. Kristen Vickers, Kristine Newman, and Jill Cameron for taking the time to share their expertise and resources with me.

This study would not at all have been possible without the invaluable contributions of several phenomenal Stress \& Healthy Aging Research Lab members. Thank you to Mitra Farzaneh for your significant role in this study - I have great respect for your attention to detail and generous spirit. I am also grateful to Vivian Huang, Shruti Vyas, and Katlyn Peck, who all offered helping hands.

Of course, I am indebted to the dozens of inspirational caregivers who took the time out of their demanding schedules to help me conduct this research. Thank you also to the individuals and organizations that helped with recruitment and gave us space to hold groups.

I am incredibly fortunate to be surrounded by such brilliant and kind peers. In particular, I want to thank Jeanine Lane, Rachel Bar, Lauren David, Hanna McCabe-Bennett, Kathleen 
Tallon, Natalie Stratton, and Andrew Brankley for their endless support, and for always cheerleading me from the sidelines. I am grateful to have you all as colleagues and friends.

This acknowledgement would not be complete without recognizing my entire family for their unconditional love and support. In particular, I'd like to thank my amazing mum, Bharati Mallya, for always modeling kindness, compassion, sincerity, and perseverance. Your encouragement has been a driving force for me throughout this dissertation and beyond. Thank you always.

And last, but certainly not least, I extend my gratitude to Tyler Toews. To know your love, strength, and laughter gives me a glimpse into the devastating loss it is for these caregivers to lose their loved ones. Thank you for being there for me in every way possible. 


\section{Dedication}

For the caregivers.

"I am devoted to helping my husband in every way and that gives me strength to provide the very best care. I do possess the skills, knowledge, and the right attitude - but of course, at the cost of my own health. He comes first. I don't always have time for myself."

\section{- Caregiver Participant}

"[I have] patience, knowledge, health; all of which are being depleted as the disease progresses."

- Caregiver Participant

"I am always looking at the bright side of things and trying to do whatever I can to look after my husband the best way I can- trying to convince myself that this is a fact of life and I need to deal with it... Always trying to feel that although the situation is bad, it could have been worse!"

- Caregiver Participant

"I have to treat my wife like small child. It does hurt me to see her this way, but I realize she is sick."

- Caregiver Participant

"I am patient (most of the time). I am physically strong, although as my husband requires more assistance, I feel my body starting to break down. I know my husband and his needs. I have read much about his disorder and I attend support group meetings. As with physical strength, my emotional strength is suffering as time goes by."

\section{- Caregiver Participant}

"I am told by family and friends that I know how to move with the times, 'ride the wave', 'remain calm', and 'smiling always'! I know for a fact that the only thing permanent in life is change... This knowledge helps me to cope with life's challenges."

- Caregiver Participant 


\section{Table of Contents}

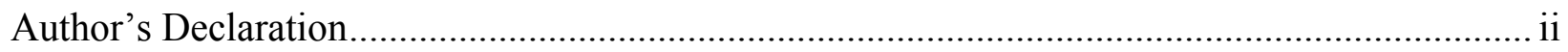

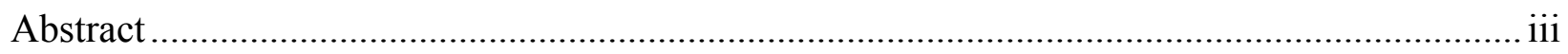

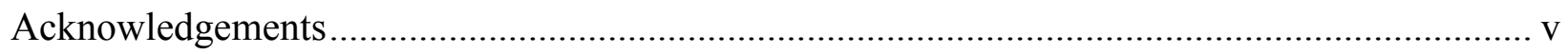

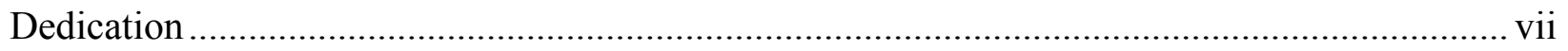

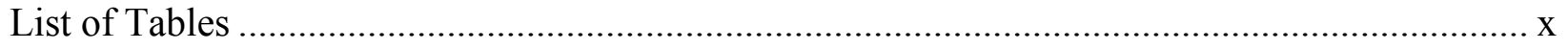

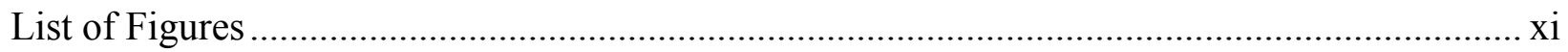

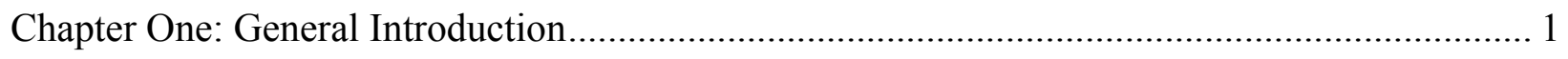

1.1. Background \& Statement of the Problem ............................................................. 1

Chapter Two: Literature Review on Neurodegeneration ...................................................... 6

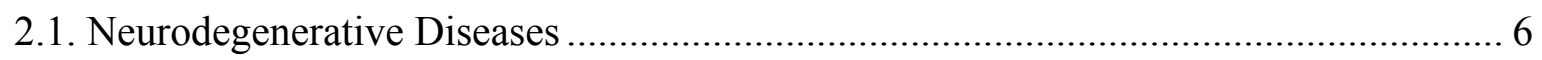

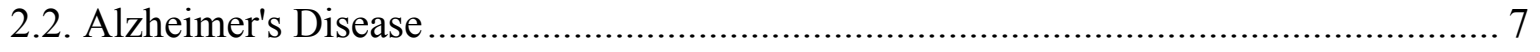

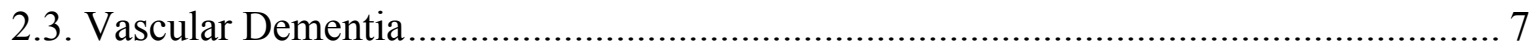

2.4. Lewy Body/Parkinson's Dementia ...................................................................... 8

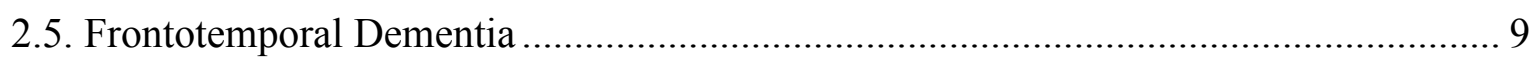

Chapter Three: Literature Review on the Informal Caregiver............................................... 11

3.1. A Broad Definition of Informal Caregiving ...................................................... 11

3.2. Why Caregivers of Persons with Dementia are Unique ..................................... 13

3.3. Characterizing Caregivers of Persons with Dementia ............................................ 16

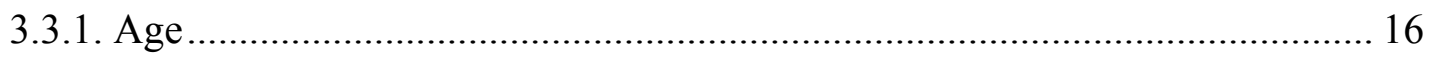

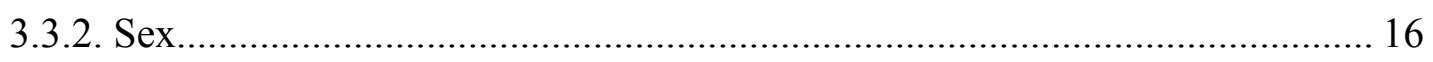

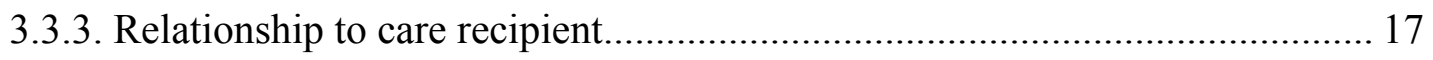

3.4. Potential Mechanism of Caregiver Burden and Health Outcomes: Chronic Stress..... 18

3.5. Cognitive Outcomes in Caregivers ............................................................... 23

Chapter Four: Interventions to Support Caregivers ........................................................ 27

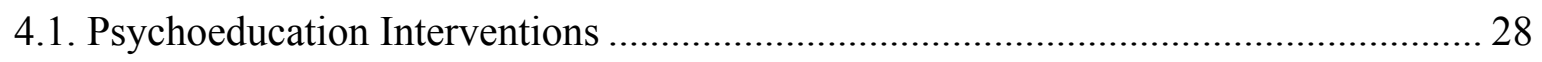

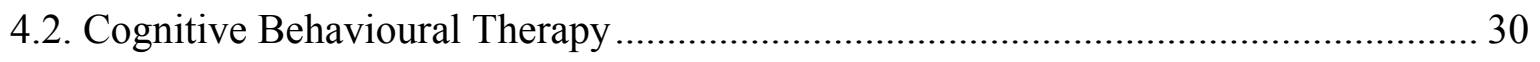

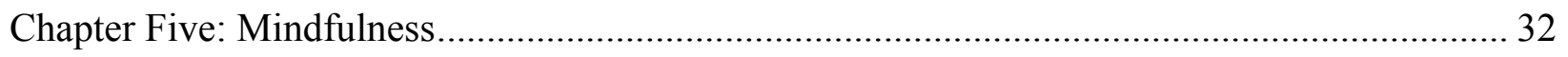

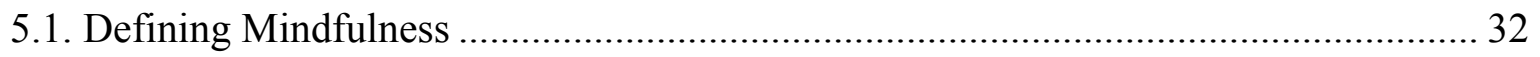

5.2. Mindfulness for Non-Dementia Caregivers..................................................... 35

5.3. Mindfulness for Caregivers of Persons with Dementia ........................................ 41

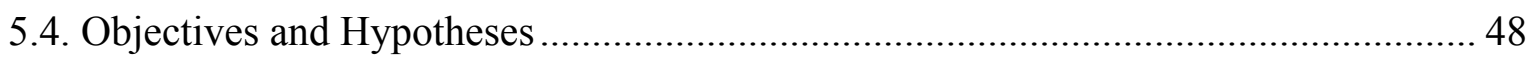




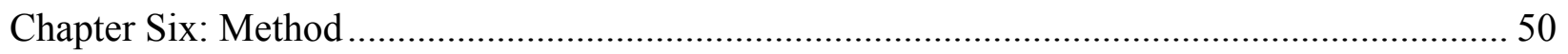

6.1. Cohort 1: Community Sample …………………………………………………. 50

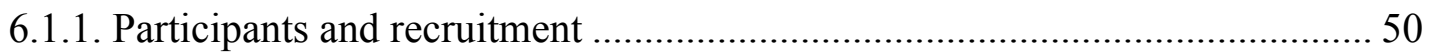

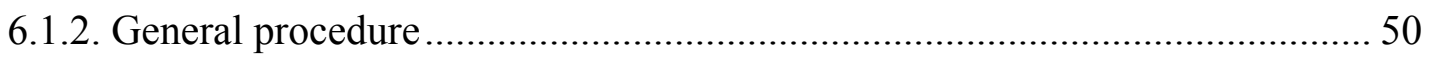

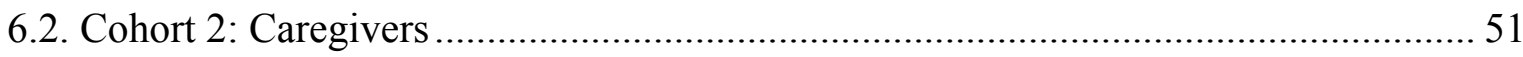

6.2.1. Participants and recruitment ………………….............................................. 51

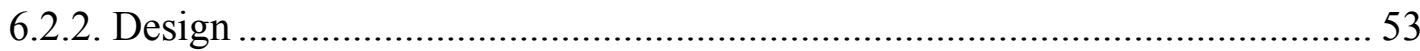

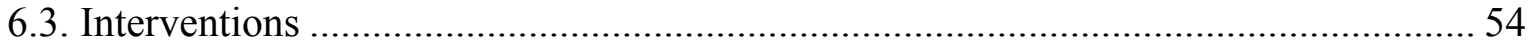

6.3.1. Mindfulness-based stress reduction ........................................................... 54

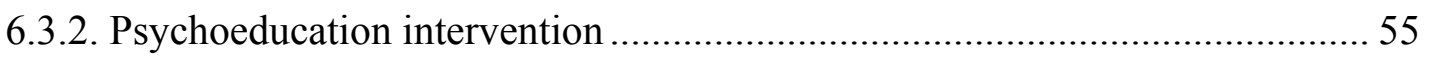

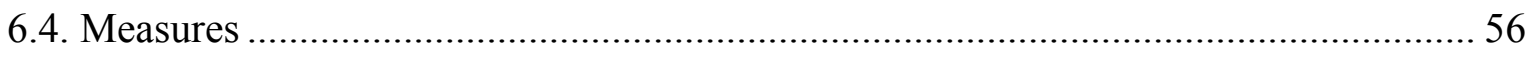

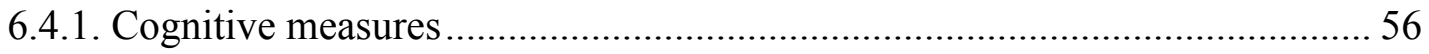

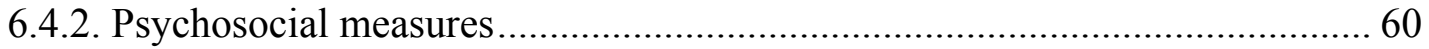

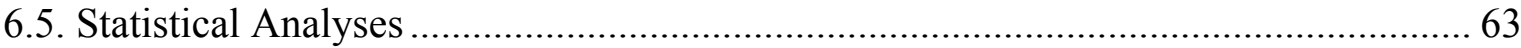

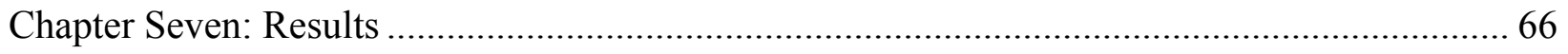

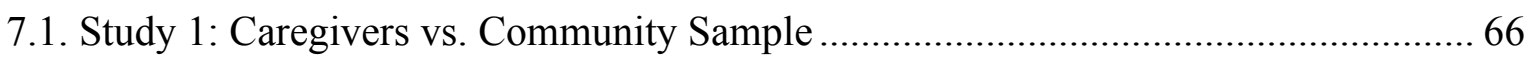

7.2. Study 2: Randomized Controlled Trial for Caregivers ............................................... 67

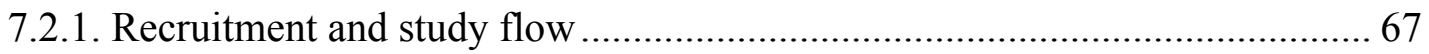

7.2.2. Baseline characteristics of the sample ............................................................ 68

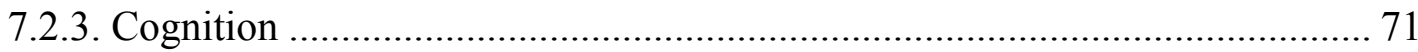

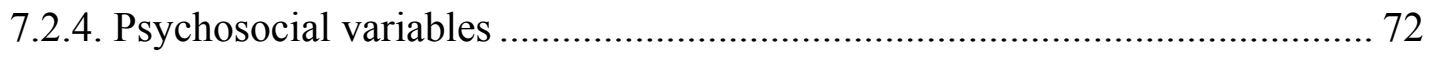

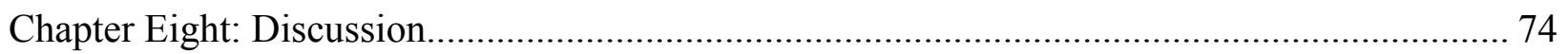

8.1. Effects of Caregiving on Psychosocial Well-Being................................................... 75

8.2. Effects of Caregiving on Cognitive Function ........................................................... 77

8.3. Effects of MBSR on Caregivers' Psychosocial Well-Being......................................... 84

8.4. Effects of MBSR on Caregivers' Cognitive Function ................................................. 88

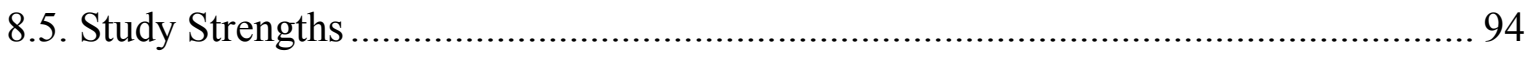

8.6. Limitations and Future Directions .......................................................................... 94

8.7. Implications and Recommendations for Further Study …………………………..... 97

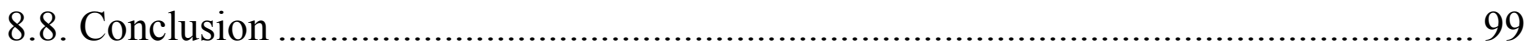

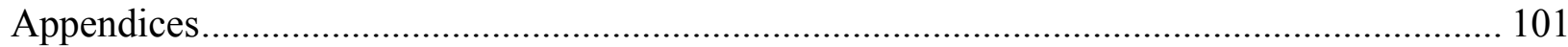

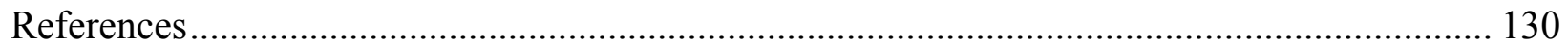




\section{List of Tables}

Table 1: Studies of Mindfulness-Based Interventions for Caregivers of Adults with Medical Disorders Unrelated to Dementia..................................................................... 37

Table 2: $\quad$ Studies of Mindfulness for Caregivers of Persons with Dementia........................... 43

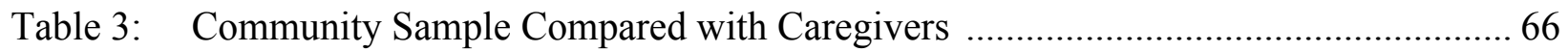

Table 4: Baseline Characteristics of Caregivers .............................................................. 70

Table 5: Comparison of Within- and Between-Group Changes Over Time: Cognitive

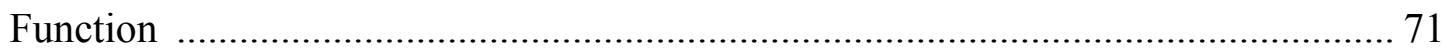

Table 6: Comparison of Within- and Between-Group Changes Over Time: Psychosocial Variables 


\section{List of Figures}

Figure 1: Schematic Representation of the Hypothalamic-Pituitary-Adrenal Axis................... 19

Figure 2: CONSORT Flow Diagram of Randomized Control Trial for Caregivers.................. 69 


\section{CHAPTER ONE}

\subsection{Background and Statement of the Problem}

The population is rapidly aging. As of July 1, 2015, the number of Canadians aged 65 years and older outnumbered the number of children aged 0 to 14 years for the first time in history (Statistics Canada, 2015). Approximately $16.1 \%$ of the population (a record 5,780,900 individuals) was at least 65 years old, compared with 5,749,400 children aged 0 to 14 years. Population projections estimate that the percentage of older Canadians will increase to $20.1 \%$ (or 1 in 5 people) by 2024 (Statistics Canada, 2015). In 2016, there were approximately 564,000 individuals living with dementia in Canada (Alzheimer Society of Canada, 2016). Given that age is the strongest known risk factor for developing a neurodegenerative disease such as dementia, the prevalence rate of dementia is expected to nearly double to 937,000 by 2031 (Alzheimer Society of Canada, 2016). The projected statistics reflect such a drastic increase in the prevalence of dementia cases in Canada that the Alzheimer Society of Canada has declared dementia to be an epidemic and has issued a national call to action.

With the rise of dementia cases in Canada comes an increase in the financial burden that this disease will place on the economy. The estimated cost of caring for people with dementia was $\$ 10.4$ billion in 2016 and this number is expected to rise to $\$ 16.6$ billion by 2031 (Alzheimer Society of Canada, 2016). Caring for someone with dementia costs $5.5 \mathrm{x}$ more than caring for someone without the disease, largely driven by the high cost of long-term care and home care (Alzheimer Society of Canada, 2016). Moreover, in addition to these direct costs, the

out of pocket expenses for informal, unpaid caregivers in 2016 was conservatively valued at $\$ 1.4$ billion and is expected to double by 2031 unless there are significant reductions in the number of people who are newly diagnosed with dementia (Alzheimer Society of Canada, 2016). 
Numerous services are available in Canada to support persons with a neurodegenerative disease and their caregivers, such as disease-specific societies (e.g., the Alzheimer Society and Parkinson's Society), health care practitioners (e.g., geriatricians, psychologists, and neurologists), community-based services (e.g., adult day programs, in-home respite care), longterm care facilities, and palliative care facilities. However, as the Alzheimer Society of Canada (2010) notes in their nation-wide study of dementia, these services are "...maldistributed, uncoordinated and, where available, delivered with little standardization and continuity" (p. 14). Marc Wortmann, the executive director of Alzheimer's Disease International, agrees. In an interview with the Globe \& Mail, Dr. Wortmann stated, "I think Canada has a lot of good services, but I'm not sure if the services are available to everyone who needs them. ...It's not enough to have services, you have to match people with the right services. In Canada, there is good care, but not enough of it, and there is no strategy" (Picard, 2011). In June 2017, the federal government of Canada implemented a national strategy to address these types of complaints (Alzheimer Society of Canada, 2017).

According to the Organisation for Economic Co-operation and Development, Canada ranks 11 th out of 11 member countries for timeliness of care (defined as access to same or next day primary care; access to after-hours care; at least two months wait for specialized care, etc.; Health Canada, 2015). For long-term care facilities specifically, older adults in Ontario waited a median of 103 days for a bed (meaning that $50 \%$ of older adults waited longer than that; Bronskill et al., 2010). It took 618 days for nine out of 10 individuals to be given a bed (Bronskill et al., 2010). Enduring such extended wait-times is especially problematic for the well-being of caregivers of persons with dementia. They generally do not seek support for their own needs until they are already experiencing high levels of burden and resentment, and their 
own health has deteriorated (Brodaty et al., 2005; Sorrell \& Cangelosi, 2009). Moreover, because projection models predict a shortfall of more than 157,000 long-term care beds by 2038 , the number of persons with dementia who will be cared for by informal caregivers in the home will grow enormously, substantially increasing the number of caregivers impacted by caregiver burden (Alzheimer Society of Canada, 2010).

Informal caregivers provide ongoing, unpaid care and assistance to relatives, spouses, and friends in need of support. Along with the rest of the population, caregivers are also getting older (Sinha, 2013). On average, caregivers aged 65 and older are reported to spend the greatest amount of time per week providing care, likely due to the enhanced commitment of caring for a spouse (Sinha, 2013). For most caregivers, caregiving is associated with a number of positive and negative experiences (Butcher, Holkup, \& Buckwalter, 2001).Caregiving can provide an opportunity to cherish a relationship, focus on joyful times, and increase commitment to the care recipient (Butcher et al., 2001). However, caregiving can also result in negative emotions including resentment and anger towards the care recipient, as well as guilt, grief, and bitterness about the role in which they have been placed (Dupuis, Epp, \& Smale, 2004).

Changes in relationship dynamics, including the loss of emotional support from the person with dementia and decreased quality of communication, can be a significant source of stress for the caregiver. For spouses, diminished marital cohesion and satisfaction due to the caregiver-receiver dynamic can have further detrimental effects on the caregiver's mental health (Baikie, 2002; Rankin, Haut, Keffover, \& Franzen, 1994). Over a decade of research shows that relationship and marital conflict is associated with poor mental health, hypertension, increased risk for cardiovascular events, and suppressed immune function (Wang, Wang, Li, \& Miller, 2014). For older adult caregivers specifically, the medical co-morbidities and functional 
limitations that are associated with aging can further exacerbate the stress of caregiving (Norton et al., 2010).

Chronic perceived stress results in chronic activation of stress response systems that are designed to be adaptive in the short term, but break down due to repeated and relentless activation (Karlamangla, Singer, McEwen, Rowe, \& Seeman, 2002). Chronic activation of stress-sensitive systems increases the individual's vulnerability to physiological dysfunction and behavioural difficulties, such as more visits to the doctor and increased reports of physical and psychological symptoms such as depression and burden (Alberts, Hadjistavropoulos, Pugh, \& Jones, 2011; Ho, Chan, Woo, Chong, \& Sham, 2009; Karlamangla et al., 2002; Markesbery, Kryscio, Lovell, \& Morrow, 2005; McEwen \& Sapolsky, 1995). Studies also show that older adult caregiving spouses display impaired cognitive function compared with non-caregiving agematched spouses (Caswell, Vitaliano, \& Croyle, 2003; Lee, Kawachi, \& Grodstein, 2004; Mackenzie, Wiprzycka, Hasher, \& Goldstein, 2009). In fact, a population-based longitudinal study found that older adult caregivers of spouses with dementia are six times more likely to develop dementia themselves compared with non-caregiving spouses, even after adjustment for age, gender, education, socioeconomic status, and genetic risk factors (Norton et al., 2010).

From a health systems perspective, there is a dual burden on the health system. More specifically, a decline in psychological and cognitive health among older caregivers not only diminishes their ability to function within the caregiver role, but also significantly increases the burden that caregivers themselves place on the health care system as they seek treatment for their own negative health outcomes. The Alzheimer Society of Canada states, "the predicted surge in dementia prevalence threatens to overwhelm Canada's health care system unless specific and targeted actions are undertaken. Canada must act" (2010, p. 14). 
Since there is no known cure for dementia, one of the Alzheimer Society's primary recommendations for moving forward is to provide interventions to support informal caregivers (Alzheimer Society of Canada, 2010). The Alzheimer Society (2010) does not provide any suggestions for specific forms of interventions, but it has issued a call-to-action to determine which methods are most effective in improving caregiver well-being. Mindfulness-based stress reduction (MBSR) is one approach that has gained considerable scientific attention in the past two decades for its potential to foster adaptive coping and resilience, and decrease perceived stress and associated health risks over time. To date, no studies have rigorously assessed the effect of a standardized MBSR intervention on psychosocial and cognitive function in older caregivers of persons with dementia and related neurodegenerative disorders.

This dissertation seeks to evaluate the utility of mindfulness training as a potential tool to improve cognitive function and decrease psychological burden associated with caregiving for a spouse or relative with a neurodegenerative disease. The sections that follow will provide an overview of the literature that supports the rationale for the current study, which sought to evaluate the utility of mindfulness as a viable intervention for caregivers of persons with dementia and related neurodegenerative disorders. This overview will begin with a review of the common types of neurodegenerative disorders that develop in older adults and require long-term care; which will then be followed by a review of the caregiver literature and common interventions that have been used for this target population; followed by a review of the literature on the benefits of mindfulness meditation and how this practice may be suitable for caregivers of persons with a neurodegenerative disease. 


\section{CHAPTER TWO}

\subsection{Neurodegenerative Diseases}

Although a large number of older adults remain healthy and independent well into late life, age is the most significant risk factor for the development of the most prevalent diseases of developed countries: cancer, cardiovascular disease, and neurodegeneration (Niccoli \& Partridge, 2012). Of these conditions, neurodegenerative diseases are the most vicious because they are incurable, progressive, and extremely debilitating (EU Joint Programme Neurodegenerative Disease Research, 2014).

Neurodegenerative diseases result from progressive degeneration and/or death of neurons in the brain and/or spinal cord, resulting in problems with mental functioning (e.g., dementia) and/or motor functioning (e.g., ataxia), depending on where the neurodegeneration is taking place (EU Joint Programme - Neurodegenerative Disease Research, 2014). These conditions cost Canadians nearly $\$ 8$ billion per year in direct (i.e., hospital care, physician care, and drug expenditures) and indirect (i.e., mortality and morbidity costs), representing about $7 \%$ of the total attributable cost of illness in Canada (Canadian Institute for Health Information [CIHI], 2007).

Neurodegenerative diseases can be broadly subdivided into two categories: those with predominantly motor symptoms and those with predominantly cognitive symptoms (i.e., dementia), although most diseases have overlapping symptoms (Hardiman \& Doherty, 2011). Examples of neurodegenerative diseases with predominantly motor symptoms include Parkinson's disease, Motor neuron disease, and Huntington's disease. The most common diseases with predominantly cognitive symptoms include Alzheimer's dementia, vascular dementia, Lewy body dementia, and frontotemporal dementia (Hardiman \& Doherty, 2011). The most commonly reported neurodegenerative disease both globally and in Canada is Alzheimer's 
disease (AD), followed by Parkinson's disease (Mayeux, 2003; Office of the Chief Medical Officer of Health, 2012).

\subsection{Alzheimer's Disease}

$\mathrm{AD}$, the most common subtype of neurodegenerative diseases, is characterized by an insidious onset of memory impairment, starting with forgetfulness for day-to-day events, and progressing to significant global memory loss such as loss of autobiographical memory or being unable to recognize loved ones (Gallagher, Mhaolaín, Sperling, \& Lawlor, 2011). Over time, persons with $\mathrm{AD}$ show progressive declines in overall cognitive function, demonstrated by difficulty communicating with others, poor decision-making ability, poor judgment, wandering and getting lost, and loss of motor skills (Gallagher et al., 2011). Caregivers must also contend with the development of significant personality changes such as apathy, depression, and agitation. Additionally, delusional thoughts related to persecution, intruders, and imposters are common, as are visual and auditory hallucinations (Gallagher et al., 2011). Functional decline (e.g., incontinence) is not uncommon and is another significant responsibility for caregivers (Gallagher et al., 2011). These symptoms generally result from an accumulation of amyloid plaques and neurofibrillary tangles in the brain. In Canada in 2011, AD was the seventh leading

cause of death (ninth among men, sixth among women; Public Health Agency of Canada, 2014).

\subsection{Vascular Dementia}

Vascular dementia arises as a consequence of an ischemic insult such as a stroke, which triggers neurodegeneration via the deprivation of oxygen and glucose to neurons (Harbison, Kennelly, \& Kenny, 2011). The clinical features of vascular dementia vary depending on the severity of the cerebral damage, but generally, include an abrupt onset of symptoms, stepwise deterioration of function, and fluctuating course of symptoms (Harbison et al., 2011). Symptoms 
often include motor abnormalities (e.g., poor reflexes and difficulty walking and stepping, which leads to falls; Briley, Wasay, Sergent, \& Thomas, 1997), focal sensory abnormalities (e.g., visual deficits), incontinence, nocturnal confusion and wandering, and depression or emotional lability (Harbison et al., 2011). Impairment of executive function (i.e., the cognitive mechanisms that control and regulate the activation of various cognitive functions; Miyake, Friedman, Emerson, Witzki, \& Howerter, 2000) is another typical feature of vascular dementia (Harbison et al., 2011). It is the second most common cause of dementia in older adults after AD. In Canada, it accounts for $14 \%$ of dementia in women and 30\% of dementia in men (Hill et al., 1996).

\subsection{Lewy Body/Parkinson's Dementia}

Lewy body dementia is typically associated with visual hallucinations and fluctuations in cognitive function (Olszewska, Fahn, Walsh, \& Lynch, 2011). It often takes a progressive and rapid course and diagnosis can only be confirmed based on the presence of Lewy bodies in the brain at autopsy. Individuals often experience periods of delirium as well as motor symptoms such as hunched posture and muscle rigidity. Given its overlap with symptoms of Parkinson's disease, it is thought that both diseases may be linked to the same underlying abnormalities in Lewy bodies. Lewy body dementia appears to be the third most frequently diagnosed form of dementia (Olszewska et al., 2011).

Parkinson's disease is a slowly progressing neurodegenerative disorder that primarily acts upon brain areas responsible for muscle movement and control (e.g., the substantia nigra and basal ganglia; CIHI, 2007). Although the cardinal clinical features are movement-based (e.g., bradykinesia, resting tremor, and postural instability; Olszewska et al., 2011), approximately $40 \%$ of individuals with Parkinson's disease also develop dementia (Olszewska et al., 2011). Parkinson's dementia and dementia with Lewy bodies have several overlapping features, such as 
forgetfulness, slowed processing speed, and difficulty concentrating, which can interfere with communication (Parkinson's UK, 2017). Initiating behaviours and planning can also be impaired, making it difficult for the individual to engage in activities of daily living such as dressing and cooking. Other symptoms include changes to appetite and sleeping patterns, apathy, significant anxiety or depression, and vivid visual hallucinations and delusions (Parkinson's UK, 2017). Significant disability typically results within 10 to 15 years of diagnosis (Olszewska et al., 2011). According to the Parkinson Society of Canada (2003), nearly 100,000 Canadians have this disease and as noted, its incidence increases with age.

\subsection{Frontotemporal Dementia}

Frontotemporal dementia is a term that encompasses a spectrum of disorders that primarily affect language and behaviour (Doherty, Hutchinson, Abrahams, \& Coen, 2011). The three major subtypes of frontotemporal dementia include: 1) progressive nonfluent aphasia, which mainly interferes with language production; 2) semantic dementia, which mainly interferes with language comprehension; and 3) behavioural variant frontotemporal dementia, which mainly affects behaviour. Changes in behaviour can include becoming noticeably withdrawn or disinhibited (e.g., exhibiting inappropriate social behaviour such as blurting something out, behaving recklessly, or loss of interest in personal hygiene). Other behavioural changes can include loss of insight into one's behaviours, overeating or compulsively putting things into the mouth, incontinence, and changes in personality (Alzheimer Society of Canada, 2015). Language changes can result in stuttering, word-finding difficulties, echolalia, difficulty keeping up with conversations, or total mutism. Symptoms are insidious in onset and progress over time. Frontotemporal dementia is the most common form of early-onset dementia (Alzheimer Society of Canada, 2015). 
It is clear how caring for someone with the above-described symptom patterns could be emotionally and physically exhausting for the caregiver. Because the incidence of neurodegenerative diseases increases with age (Mayeux, 2003), the burden of caring for and living with someone with dementia or related neurodegenerative disease will only increase over time. Considering the anticipated growth in the number of neurodegenerative disease cases, it is essential to conduct empirical investigation into the burden that these diseases will place on the Canadians who care for them. Given that an estimated $90 \%$ of persons with dementia who continue to live at home are provided with 'informal' care by family and/or friends (Angus, Auer, Cloutier, \& Albert, 1995; Cantor, 1991), the current dissertation will specifically address literature relevant to informal caregivers. 


\section{CHAPTER THREE}

\subsection{A Broad Definition of Informal Caregiving}

An informal caregiver has been defined as a person who provides extraordinary, unpaid care to someone, mainly in the home setting, involving significant amounts of time and energy, and requiring engagement in tasks that may be physically, emotionally, socially, and/or financially demanding (Biegel, Sales, \& Schulz, 1991). Approximately 13 million Canadians (46\% of the population aged 15 and older) have provided informal care to a family member or friend with a long-term health condition, disability, or age-related condition (Sinha, 2013). In Ontario specifically, nearly a third (29\%) of residents have cared for an ill family member or friend, which represents a rate that is higher than the national average of $28 \%$ (Sinha, 2013).

Informal care contributes significantly to Canadian economy and society. As reviewed by Fast (2015), the Organisation for Economic Co-operation and Development estimates that the Canadian informal care sector is more than 10 times the size of the formal care workforce (Columbo et al., 2011), while the Conference Board of Canada similarly reports that informal caregivers aged 45 and older in Canada spent 15.5 billion hours of care in 2007; a figure 10 times greater than the number of formal care hours provided in the same year (Hermus et al. 2012). Recent estimates value informal caregiving for older adults at between $\$ 25$ billion and \$26 billion in 2009, representing as much as 2 percent of the Canadian gross domestic product that year (Hollander, Liu, \& Chappell, 2009).

Thus, the unpaid informal care that Canadians provide to relatives, spouses, and friends, benefits not only the care recipients, but also Canada's economy and society as a whole (CIHI, 2010; Hermus, Stonebridge, Theriault, \& Bounajm, 2012). Yet, because the labour is unpaid, its economic value and its societal contribution are often disregarded. Moreover, the cognitive, 
psychological, and physical consequences of caregiving are often overlooked (Fast, 2015).

Informal caregivers are often unprepared and untrained to take on caregiving responsibilities when the role is placed on them. As described in a qualitative study of informal caregivers of persons with dementia in Canada, caregivers struggle with learning medical terminology and skills related to diagnoses, symptoms, and treatments (Lilly, Robinson, Holtzman, \& Bottorff, 2012). Unlike professional caregivers such as nurses, who undergo extensive theoretical and practical training in caring for someone with a neurodegenerative disease, informal caregivers receive no systematic training in helping with activities such as toileting and bathing, wound care, feeding, and changing catheter bags. They report receiving inadequate support from health care providers and feeling abandoned by the health care system (Lilly et al., 2012). In contrast to formal caregivers, it is also likely that informal caregivers had no innate desire to assume a caregiving role. Moreover, some informal caregivers (spouses in particular) may be exposed to the care recipient for 24 hours per day, 7 days a week without ever getting a break (Adelman, Tmanova, Delgado, Dion, \& Lachs, 2014). For many caregivers, making important short- and long-term legal, financial, and housing-related decisions will be a new experience. For some spousal caregivers, these types of decisions will have to be made without their spouse, who may have been their main source of support. Lastly, in contrast to formal caregivers, informal caregivers will have to manage these responsibilities while simultaneously coping with the significant emotional loss that accompanies watching a loved one deteriorate.

These caregiving responsibilities may make it more difficult for the caregiver to additionally manage his or her other responsibilities, such as employment, child rearing, selfcare, and maintaining interpersonal relationships. Having to balance these multiple roles is 
presumably a very stressful task. When asked, $28 \%$ of caregivers in Canada found caregiving to be "somewhat" or "very" stressful (Sinha, 2013). More than half (55\%) of caregivers surveyed reported feeling worried or anxious as a result of their caregiving responsibilities, while $36 \%$ reported feeling short-tempered or irritable and 35\% reported feeling overwhelmed (Sinha, 2013).

The negative effects of caregiving have led caregivers to be described as the "hidden patient" (Fengler \& Goodrich, 1979). In addition to the high levels of perceived stress associated with caregiving, caregivers are also more likely to experience pain and chronic disease than noncaregivers (Ho et al., 2009); long-term sleep disturbances (McCurry, Logsdon, Teri, \& Vitiello, 2007); hypertension (Capistrant, Moon, \& Glymour, 2012); and coronary heart disease and stroke (Ji, Zoller, Sundquist, \& Sundquist, 2012). Caregiving is further associated with a 63\% higher chance of death relative to non-caregivers (Schulz \& Beach, 1999). In light of these sobering statistics, it is important to understand the factors that place caregivers at such high risk for negative outcomes. Several reports suggest that the care recipient's diagnosis is one such risk factor.

\subsection{Why Caregivers of Persons with Dementia are Unique}

The adult caregiving literature has largely been divided into two main areas: caregivers for persons with a medical diagnosis unrelated to dementia (e.g., cancer), and caregivers for persons with dementia and related neurodegenerative diseases. Caring for a loved one with cancer is certainly related to significant distress. However, when comparing cancer caregivers to caregivers of persons with dementia, spousal caregivers of persons with cancer are on average younger (as are their care recipients); significantly more likely to maintain employment; and have been caregivers for significantly shorter periods of time (Clipp \& George, 1993). Each of 
these characteristics is presumed to be protective for the caregiver. In a study comparing cancer caregivers to caregivers of persons with dementia on several measures of well-being, caregivers of persons with dementia showed poorer outcomes on 16 of 19 measures of physical health, substance use, emotional health, social life, and financial status, even after adjusting for disease symptoms (Clipp \& George, 1993). In both groups, age was the most robust and powerful predictor of well-being, with older caregivers showing worse outcomes (Clipp \& George, 1993).

Given that neurodegenerative diseases are age-related (Byrne et al., 2011), caregivers for someone with one of these diseases are generally older than non-neurodegenerative disease caregivers and thus, they are at increased risk of negative outcomes (Clipp \& George, 1993). Although there are numerous neurodegenerative disorders within this diagnostic classification, the vast majority of the caregiving literature in older adults has focused on dementia specifically. As a result, this review will report on literature relevant to dementia care rather than neurodegenerative care in general.

Research findings consistently show that relative to caregivers of persons without dementia, caregivers of persons with dementia report significantly poorer health and well-being. For instance, Ory, Hoffman, Yee, Tennstedt, and Schulz (1999) surveyed 1,509 racially stratified individuals $(M$ age $=46$ years $)$ across the United States who reported providing unpaid care to a relative or friend who was at least 50 years of age. The caregivers were divided into two groups: 1) those who reported caring for someone with either AD, confusion, dementia, or forgetfulness (i.e., the 'dementia' caregivers); and 2) those who reported providing care to someone without a neurodegenerative disease (i.e., the 'non-dementia' caregivers). All participants were asked to answer questions about the amount and type of care they provided and how caregiving impacted their lives. After controlling for intensity of caregiving involvement and relevant 
sociodemographic factors, results demonstrated that compared with the non-dementia caregivers, the dementia caregivers reported significantly greater employment complications, caregiver strain, mental and physical health problems, interference with time spent on leisure activities, and interpersonal conflict (Ory et al., 1999).

Depression is also nearly twice as common in caregivers of persons with dementia as in other caregivers (Canadian Study of Health and Aging [CSHA], 1994). According to the CSHA (1994), symptoms of depression are reported in $16 \%$ of informal caregivers assisting someone with mild dementia at home, $40 \%$ of informal caregivers caring for someone with moderate dementia at home, and $18.4 \%$ of informal caregivers assisting someone with severe dementia in an institution.

Other studies show that relative to caregivers of adults with other medical diagnoses, caregivers of persons with dementia report spending more time providing care (Desbiens, Mueller-Rizner, Virnig, \& Lynn, 2001; Ory et al., 1999), helping with more basic and personal activities of daily living (ADL) and instrumental activities of daily living (iADL; e.g., Pinquart \& Sörensen, 2003), and responding to behavioural problems (e.g., Hooker et al., 2002; ShanksMcElroy \& Strobino, 2001). A meta-analysis of 228 studies conducted by Pinquart and Sörensen (2003) examined the aspects of caregiving related to perceived burden. demonstrated significant positive correlations between caregiver burden and cognitive impairment of the care recipient, hours of care provided per week, and number of caregiving tasks performed. They also showed that compared with any other variable, the care recipient's behavioural problems had the strongest association with caregiver burden. All of these aspects of caregiving were most commonly reported by caregivers of persons with dementia.

Moreover, caregivers for persons with dementia report more health problems, less life 
satisfaction, more overload, more familial conflict, more anger, and more use of psychotropic medication than caregivers of older people with physical impairments without dementia (Livingston, Manela, \& Katona, 1996; Morris, Morris, \& Britton, 1988; Schofield, Murphy, Herrman, Bloch, \& Singh, 1998; Schulz, O’Brien, Bookwala, \& Fleissner, 1995; Vitaliano, Young, \& Zhang, 2004). Considering the significant impact of caregiving for someone with dementia, it is not surprising that these caregivers have a greater need for formal services (Ory et al., 1999).

\subsection{Characterizing Caregivers of Persons with Dementia}

3.3.1. Age. The average age of Canadian caregivers for someone with dementia is 48 years (Alzheimers Association, 2009), but an increasing number of caregivers are aged 65 and older (Dupuis, Epp, \& Smale, 2004). Indeed, the Canadian Study on Health and Aging (CSHA) notes that $34 \%$ of caregivers are at least 70 years of age, while $11 \%$ are at least 80 years of age (CSHA, 1994). Married men who are caregivers are, on average, 73 years of age (Keating, Fast, Frederick, Cranswick, \& Perrier, 1999). Given that caregivers of persons with dementia are commonly older adults themselves, it is important to highlight how the effects of aging can compound the already deleterious effects of being a caregiver for someone with dementia. Some studies have shown that older adults perceive higher levels of caregiver burden than younger adults because they themselves may be experiencing physical impairments, medical comorbidities, and less social support (Chien, Chan, \& Morrissey, 2007; Watson, Modeste, Catolico, \& Crouch, 1998). The physical and emotional demands associated with caregiving may further influence changes in health status in older adult caregivers (Greene, Taylor, \& Johnson, 1993).

3.3.2. Sex. The CSHA (1994) reported that approximately $70 \%$ of caregivers of persons 
with dementia are female, typically wives (24\%) or daughters $(29 \%)$. Although some studies have demonstrated that sex is unrelated to care burden (Rezende, Coimbra, Costallat, \& Coimbra, 2010), several other studies have shown that females were more likely to report burden, and reported greater burden than males (Akpinar, Küçükgüçlü, \& Yener, 2011; Gallicchio, Siddiqi, Langenberg, \& Baumgarten, 2002). In Canada, female caregivers are significantly more likely than males to visit or call to ensure the care recipient is okay, provide emotional support, conduct household work, make appointments, and be involved with medical treatments and personal care (Sinha, 2013). A meta-analysis conducted by Pinquart \& Sörensen (2006) integrated results from 229 studies to examine how gender affects caregivers of individuals with dementia. The caregivers had a mean age of 59.5 years $(S D=8.0$ years $)$. They found that women had higher levels of burden and depression, and lower levels of subjective well-being and physical health relative to men. However, Pinquart and Sörenson (2006) also state that most of these gender differences were small or very small in magnitude. Nonetheless, the authors concluded that women experience more caregiving stressors, which increases gender differences in depression and physical health among caregivers.

3.3.3. Relationship to Care Recipient. In Canada, caregivers for older adults with dementia are most often spouses (36\%), daughters (28\%), or sons (9\%; Cohen, Pringle, \& LeDuc, 2001). Of note, the proportion of spousal caregivers may be somewhat low, as a result of possible underreporting (Cranswick \& Dosman, 2008); spouses may not view responsibilities as “caregiving" activities per se, but rather view the caregiving duties as an extension of their role as a spouse. Spousal caregivers have been shown to provide the most intense level of assistance to an older adult with dementia (Keating et al., 1999), with some results suggesting that spouses provide three to five times more care than other family members (Frederick \& Fast, 1999). 
Spouses are also more likely to provide assistance with a broader range of tasks, and for a longer period of time than adult children (Pruchno \& Potashnik, 1989). Relative to sons, adult daughters are three times more likely to provide assistance with ADLs and are twice as likely to provide assistance with iADLs (Lee, Dwyer, \& Coward, 1993). Vitaliano et al. (2002) found differential patterns of symptom presentation among caregiving spouses, such that wives reported greater caregiver burden, whereas husbands were more likely to develop heart disease and metabolic syndromes, a physical manifestation of chronic stress.

Considering that a vast body of literature consistently suggests that caregiving is related to negative health outcomes, it is important to understand the underlying processes that contribute to these negative outcomes. Vitaliano, Zhang, and Scanlan (2003) suggest two pathways that may underlie the relationship between caregiving and negative health outcomes. One pathway is related to the way in which psychosocial stress leads to poor health habits such as poor diet and sedentary lifestyle. The other pathway is related to processes of the physiological stress system and its effects on physical, psychological, and cognitive function.

\subsection{Potential Mechanism of Caregiver Burden and Health Outcomes: Chronic Stress}

When faced with a stimulus that is initially appraised as stressful, harmful, or threatening (e.g., being confronted by a loved one with dementia who is exhibiting disruptive behaviour), the feelings of distress can lead to activation of the physiological stress response systems. The two primary stress response systems are the sympathetic-adrenal-medullary (SAM) system and the hypothalamic-pituitary-adrenal (HPA) axis (Herman \& Cullinan, 1997). Activation of these stress response systems can be described as occurring in two waves. The response of the SAM system is the primary, fast acting wave that prepares the body to deal with the stressor in the short-term (i.e., the fight-or-flight response). Sympathetic activation of 
the adrenal medulla leads to almost immediate secretion of norepinephrine (NE) and epinephrine (EPI; Thierry, Javoy, Glowinski, \& Kety, 1968), which leads to increased heart rate, respiration, blood flow, and blood pressure, while simultaneously suppressing digestion, growth, and the reproductive and immune systems (Sapolsky, 1999).

The second, slower wave consists of HPA axis activation, which takes minutes to respond but remains activated for a prolonged period lasting from minutes to hours (McEwen \& Sapolsky, 1995). Activation of the HPA axis begins with the secretion of corticotropinreleasing hormone (CRH; Herman \& Cullinan, 1997) from the paraventricular nucleus (PVN) of the hypothalamus, which stimulates the anterior pituitary gland to secrete and release adrenocorticotropin hormone (ACTH), which enters the bloodstream and activates the adrenal cortex, resulting in the culmination of glucocorticoids (GC; Hennessy \& Levine, 1979), or cortisol in humans. Cortisol raises heart rate and blood pressure, which serve to increase the availability of blood glucose (i.e., energy) to the muscles (Sapolsky, 1999). Cortisol is also vital for turning the HPA system off through a negative feedback inactivation system, signaling the hypothalamus and adrenal glands to decrease hormone secretion (Figure 1). Although this biological stress response is adaptive under conditions of acute stress, excessive exposure to these stress hormones can affect the efficiency of the HPA axis.

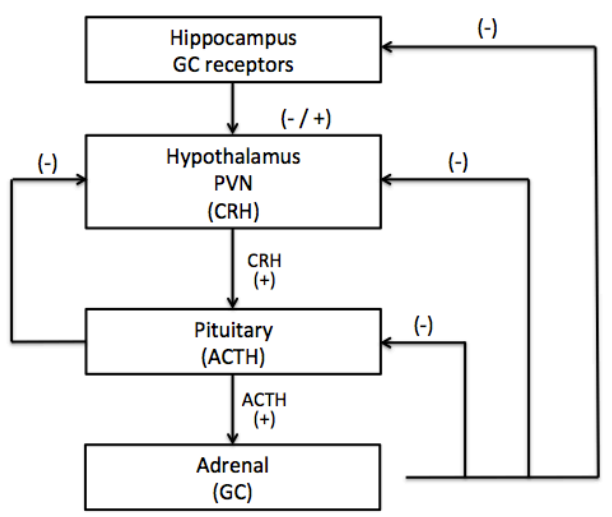

Figure 1. Schematic representation of the HPA axis. Adapted from Fiocco (2007). 
Studies on informal caregivers, who typically experience prolonged significant physical, psychosocial, and monetary stress (Pinquart \& Sörensen, 2003; Schulz \& Martire, 2004), provide evidence for the biological effects of chronic stress. For instance, stressed caregivers show increased levels of salivary cortisol throughout the day (Bauer et al., 2000; Gallagher-Thompson et al., 2006; Vedhara et al., 1999) and increased plasma NE levels (Mills et al., 1997). Compared with healthy controls, caregivers have also been found to have alterations in HPA functioning, demonstrated by lower dehydroepiandrosterone sulfate (DHEAS; the metabolite of dehydroepiandrosterone [DHEA], a cortisol antagonist), higher cortisol-to-DHEAS ratio, and less sensitivity to the HPA negative feedback loop (Jeckel et al., 2010).

In a meta-analysis, Vitaliano et al. (2003) integrated the results of 23 studies that compared the physical health of caregivers of persons with dementia with age- and sex-matched control non-caregivers. They found that caregivers had a $23 \%$ higher level of stress hormones and a $15 \%$ lower level of antibody responses than did non-caregivers. Although the authors caution that these observational data do not definitively suggest that caregiving 'causes' illness, a number of observational studies have shown that excessive exposure to stress hormones (e.g., while providing full-time, ongoing care to a loved one) is related to compromised immune function (Mausbach et al., 2008), increased blood pressure and risk of coronary heart disease (von Känel et al., 2008), increased depression (Bergdahl \& Bergdahl, 2002), decreased overall well-being (Cohen, 1986), and impaired brain function (Lyons, Lopez, Yang, \& Schatzberg, 2000).

In addition to exerting effects on the body, stress hormones also affect cognitive functioning. In the course of the HPA feedback loop, cortisol crosses the blood brain barrier, 
where it acts on various brain regions including the hippocampus (McEwen \& Sapolsky, 1995) and the prefrontal cortex (PFC; Starcke \& Brand, 2012). These regions have a dense concentration of cortisol receptors and are implicated in learning and memory, attention, and executive functions (Lupien \& Lepage, 2001). According to the glucocorticoid hypothesis, lifetime overexposure to cortisol due to chronic perceived stress can lead to neuronal atrophy, and ultimately, result in cognitive impairment (Sapolsky, Krey, \& McEwen, 1986). This model has been refined over time (e.g., Garrido, De Blas, del Arco, Segovia, \& Mora, 2010; Swanwick et al., 1998) and it is now more widely accepted that chronic exposure to cortisol over the lifespan is not the sole determinant of neuronal atrophy and cognitive decline; rather, cortisol serves to increase the vulnerability of the hippocampus and frontal lobes to subsequent insults as well as normal wear and tear over time (Conrad, 2008).

Hippocampal-dependent learning and memory rely on ongoing synaptic strengthening and communication, and disruption of this process hinders learning and memory formation (Sapolsky, 1999). Although brief periods of mild stress encourage synaptic growth, major episodes or chronic periods of cortisol exposure inhibit this process, thereby impeding learning and memory abilities (Lupien et al., 1998). Both animal and human models support this idea. For instance, Woolley and colleagues (1990) demonstrated that exogenous administration of glucocorticoids leads to neuronal atrophy in rats; specifically, they found a decrease in the apical dendritic length of CA3 pyramidal neurons. Restraint stress and social stress have also resulted in similar effects in both rodent (Watanabe et al., 1992) and nonhuman primate models (Uno et al., 1989).

Similar findings, although correlational in design, have been reported in human research. Among individuals with Cushing's syndrome, a disorder characterized by elevated 
levels of cortisol, hypercortisolemia is negatively correlated with hippocampal volume (Starkman et al., 1992). Older adults with AD also have high levels of circulating cortisol (Jenike \& Albert, 1984) and present with reduced hippocampal volume (Du et al., 2001). One prospective longitudinal study demonstrated that among healthy post-menopausal women, increased exposure to chronic life stress over 20 years predicted later reductions in hippocampal grey matter (Gianaros et al., 2007). In both animal and human models, including healthy older adults (Lupien et al., 1998) where high levels of cortisol are observed, smaller hippocampal volume is associated with impairments in learning and memory.

The PFC is largely responsible for higher order thinking abilities (i.e., executive functions), such as attention, working memory, task-switching, verbal fluency, and decisionmaking (Lie, Specht, Marshall, \& Fink, 2006). As mentioned, the frontal lobes are thought to be particularly vulnerable to the detrimental effects of chronic stress, given the high concentration of glucocorticoid receptors in this region (McEwen, 1998). Both animal and human models have demonstrated a negative correlation between PFC grey matter volume and exposure to chronic stress (Gianaros et al., 2007; Radley \& Morrison, 2005). Human studies generally produce consistent findings that chronic stress impairs PFC functioning, as demonstrated by impairments in working memory (Lewis, Nikolova, Chang, \& Weekes, 2008), selective attention (Skosnik, Chatterton, Swisher, \& Park, 2000), and decision-making (Porcelli \& Delgado, 2009).

The risk of developing stress-related cognitive impairment only increases when age is factored in, as even healthy aging is generally associated with a certain amount of cognitive decline (e.g., slower and less efficient information processing speed; Jolles, 1986). Aging is also associated with increases in catabolic hormones (i.e., energy-consuming hormones) such 
as cortisol, and significant decreases in anabolic hormones (i.e., energy-producing hormones) including DHEA and DHEAS, testosterone, estradiol, growth hormone, and insulin-like growth factor-1 (Meerlo, Sgoifo, \& Suchecki, 2008). Lupien et al. $(1994,1998)$ showed that in older adults, individuals with chronically high levels of cortisol display greater declines in selective attention, reduced memory, and hippocampal atrophy relative to older adults with normal cortisol levels. Similarly, Lee et al. (2007) demonstrated that in a sample of 1140 adults aged 50 to 70 years, higher levels of cortisol were associated with worse performance on measures of language, processing speed, executive function, verbal memory and learning, and visual memory.

\subsection{Cognitive Outcomes in Caregivers}

Only recently have scientists begun to empirically investigate the effects of caregiving on cognitive functioning. Although a wide variety of measures have been used to assess cognitive function, results consistently show patterns of decline. Vitaliano et al. (2005) recruited 96 caregivers of spouses with $\mathrm{AD}$ and compared their performance on a measure of vocabulary to 95 matched non-caregiver spouses. Although both groups were comparable at the start of the study, caregivers showed significant decline in verbal recognition relative to noncaregivers over the course of 2 years. Caswell et al. (2003) showed that older adult spousal caregivers of persons with $\mathrm{AD}(M$ age $=74.27, S D=7.91)$ performed significantly worse than demographically-matched non-caregiving spouses on a measure of processing speed and attention (i.e., the Digit Symbol subtest of the Wechsler Adult Intelligence Scale-Third Edition [WAIS-III], now referred to as the Coding subtest in the WAIS-IV). Of note, this study was limited by cross-sectional design, as cognitive performance was only measured once. Thus, decline could not be tested. 
To address this limitation, Vitaliano et al. (2009) also administered the Digit Symbol task to spousal caregivers of persons with dementia, but looked at the data longitudinally. The authors compared 122 caregiving spouses with 117 demographically matched non-caregiver spouses at enrolment, 1 year later, and 2 years after enrolment. Results not only showed that caregivers had slower processing speed at all time points than non-caregivers, but also reported that caregivers' performance declined 4.5 times faster than non-caregivers. These results have significant implications because the Digit Symbol task predicts problem solving and ability to carry out functions necessary for independent living (Willis, Jay, Diehl, \& Marsiske, 1992), which are essential skills when caring for someone with dementia at home.

Vitaliano et al. (2009) further demonstrated that these results were mediated by depressive symptoms in the caregivers only. Similarly, Caswell et al. (2003) demonstrated that self-report burden mediated cognitive scores in caregivers only and not in controls. Thus, both Caswell et al. (2003) and Vitaliano et al. (2009) suggest that psychotherapeutic interventions that reduce distress may have an additional benefit of improving caregiver cognitive functioning. Caswell et al. (2003) specifically emphasize that their findings support previous research that suggests that, "objective stressors (e.g., caregiving) may only set the stage for adverse outcomes, but one's subjective responses (i.e., perceived distress in response to stressors) are what provide the pathway between stress exposures and deleterious outcomes" (Cohen, Evans, Krantz, \& Stokols, 1986, p. 312).

Lee, Kawachi, and Grodstein (2004) also conducted a study examining the effects of caregiving stress on cognitive function, but administered a more comprehensive battery of cognitive tests than Caswell et al. (2003) and Vitaliano et al. (2005, 2009). Specifically, they administered a cognitive screening measure as well as measures of immediate and delayed 
verbal recall, verbal fluency, and working memory. All tests were telephone-based and were administered to 13,740 females aged 70-79 years, who were participating in the Nurses' Health Study. They found that women who self-identified as informal caregivers had 20-30\% higher risks of poor performance on the cognitive screening measure, immediate recall of a 10 -word list, and overall cognitive function (based on a combination of all measures) than the noncaregivers. The authors state that these data suggest that caregiving is related to a modest risk of low cognitive function in older women. Of note, this study was limited by the restrictive inclusion of registered nurses, the cross-sectional design, and neglecting to assess physiological mediators of cognitive function. Nonetheless, these results were replicated in studies by de Vugt et al. (2006), who found that relative to controls, caregivers performed significantly worse on measures of delayed recall, information processing speed, and cognitive flexibility; and Mackenzie, Smith, Hasher, Leach, and Behl (2007), who found relative impairments in attention and aspects of memory performance.

In a landmark study, Norton et al. (2010) expanded on these findings: they conducted a population-based longitudinal study that found that older adult caregivers of spouses with dementia are six times more likely to develop dementia themselves compared with noncaregiving spouses, even after adjustment for age, gender, education, socioeconomic status, and APOE genotype (Norton et al., 2010). The risk of developing dementia jumped from $48 \%$ to $600 \%$ when the amount of time that spouses were exposed to their spouses' dementia was included in the analyses. Thus, in addition to age, another significant mediator of the relationship between caregiving and subsequent dementia may be duration of exposure to living with a dementing partner. If this is the case, then older adults are particularly at risk for the deleterious cognitive outcomes of stress. 
Overall, caregiving has consistently been found to be associated with poor outcomes. This is worrisome, as caregivers are often required to perform complex and cognitively demanding tasks in their caregiver role (e.g., manage timing of medication, keep appointments etc.; Schulz \& Martire, 2004). Unfortunately, the negative outcomes of caregiving do not appear to subside when caregiving responsibilities have ended. Esterling, Kiecolt-Glaser, Bodnar, and Glaser (1994) examined the differences between active caregivers, former (bereaved) caregivers, and non-caregiving controls. The findings showed that although over two years on average had elapsed since the former caregivers' spouse had died, this group was indistinguishable from active caregivers on depressive symptomatology and perceived distress. Moreover, both active and bereaved caregivers reported significantly more stress and depressive symptoms than the controls with no history of caregiving (Esterling et al., 1994).

In summary, the global prevalence of dementia is expected to double every 20 years (World Health Organization, 2012), which will ultimately increase the demand for informal care. As a result, the creation of prevention programs targeted at maintaining health and well-being in caregivers is imperative, particularly because it appears that subjective responses to objective stressors are what drive the relationship between stress exposure and negative outcomes (Caswell et al., 2003; Cohen et al., 1986). While not all caregivers will experience accelerated cognitive decline, these alarming statistics call for the implementation of programs that may prevent decline in cognitive and mental health. Addressing the need for healthcare interventions in this population will not only enable caregivers to effectively function in their role, but will further minimize the potential economic burden associated with treating stress-related illness. 


\section{CHAPTER FOUR}

A number of interventions to help caregivers manage their responsibilities and decrease their perceived burden have been designed and evaluated. In a meta-analysis, Sörensen, Pinquart, and Duberstein (2002) reviewed 78 studies of interventions for informal caregivers of older adults to evaluate their effectiveness on six outcome variables: caregiver burden, depression, subjective well-being, ability and knowledge about caregiving, and care recipient symptoms. Based on their review, the authors suggest that caregiver interventions can be divided into two major groups: a) those aimed at reducing the objective care burden of caregivers (e.g., respite, enhancing competence of the care recipient); and b) those aimed at improving the caregiver's well-being (e.g., psychoeducation, psychotherapy). They further break these two groups into six distinct types of interventions: psychoeducation, social support interventions, respite/adult day care, psychotherapy, interventions based on enhancing the care recipient's competence, and multicomponent interventions (Sörensen et al., 2002).

In terms of intervention efficacy, Sörensen et al.'s (2002) results showed that psychotherapy was the only intervention that had significant positive effects on all six outcome variables. More specifically, in addition to the positive impact that psychotherapy interventions had on the caregiver-specific outcomes (i.e., burden, depression, subjective well-being, and ability/knowledge), they also positively impacted the care recipient's symptoms. The authors propose that psychotherapy may have taught the caregiver how to respond more effectively to the care recipients' difficult behaviours and emotions, thus improving the well-being of the care recipients themselves.

Results of this meta-analysis also showed that psychoeducational interventions had some of the lowest dropout rates and had significant effects for all outcomes except well-being and care recipient symptoms. The other interventions were only effective in two to three outcome 
variables. Interventions with older caregivers yielded larger improvements of burden, subjective well-being, ability/knowledge, and care receiver symptoms than those with younger caregivers (Sörensen et al., 2002).

Sörensen et al. concluded that in order to effect change in the largest number of quality of life factors, psychotherapy and psychoeducational interventions may be two of the best options for caregivers. They also suggest that interventions of 7-9 sessions should be adequate to increase caregiving ability/knowledge, and that interventions should focus on trying to influence the affective aspects of caregiving in addition to the behavioural aspects. Lastly, they recommend that intervention studies should include older caregivers, as they often have more risk factors for distress such as shrinking social networks, lower income, personal health problems, and may need increased assistance with building social networks, accessing respite care, and taking care of their own health, relative to younger caregivers. Thus, they recommend increased emphasis on group-based interventions that may help older caregivers build supportive social networks (Sörensen et al., 2002).

\subsection{Psychoeducation Interventions}

Sörensen et al. (2002) point out that one of the most consistently efficacious interventions for caregivers is psychoeducation. Based on their review of 38 studies that tested a psychoeducational intervention for caregivers, they define psychoeducational interventions as those that involve a structured program aimed at providing information about the care recipient's disease process, resources and services, and teaching caregivers to respond effectively to their care recipient's symptoms, such as memory and behaviour problems (Chiverton \& Caine, 1989;

Ostwald, Hepburn, Caron, Burns, \& Mantell, 1999, Schultz, Smyrnios, Schultz, \& Grbich,1994). They state that psychoeducational interventions typically involve a combination of lectures, 
group discussions, and written materials, and must be led by a trained facilitator. Lastly, Sörensen et al. (2002) state that although social support may be a part of a psychoeducational group, it should be secondary to the educational content. The most widely used interventions for caregivers are psychoeducational in nature and combine social support with caregiver education (Acton \& Kang, 2001).

A number of studies have tested the efficacy of psychoeducational interventions for caregivers. Although specific session-by-session intervention characteristics are not typically reported in these studies, the overall structure and themes of the interventions contain several similarities. For instance, in a study that evaluated the effects of a psychoeducational group intervention for adult children caring for aging parents, Schwiebert and Myers (1994) described their four session psychoeducational intervention as follows: the first session discussed agerelated physiological changes and the effect of these changes on the aging persons; the second session focused on effective communication skills; the third session focused on stress reduction, time management, and self care; and the last session was about Eriksonian stages of development.

Ostwald, Hepburn, Caron, Burns, and Mantell (1999) tested a psychoeducational intervention that provided caregivers with information about dementing diseases; practical skills for dealing with caregiving tasks; techniques to strengthen caregivers' feelings of confidence and competence; and communication skills. The psychoeducational intervention for caregivers tested by Brodaty, Roberts, and Peters (1994) consisted of themes related to education about the disease process; strategies for coping with behavioural problems; communication skills; and stress management. Lastly, the Powerful Tools for Caregivers intervention (Schmall, Cleland, \& Sturdevant, 2000) addressed issues related to caregiving challenges and self-care; stress 
management; effective communication; learning to listen and deal with emotions such as guilt; and managing tough decisions (Schmall et al., 2000).

In summary, these psychoeducation interventions most typically included information about the care recipient's disease process; accessing resources and services; effective communication; time management; self-care; and stress management techniques (e.g., exercise, relaxation strategies). As described earlier, psychoeducation interventions generally result in improvements in caregiver burden, depression, and ability and knowledge about caregiving, but importantly, do not affect the caregiver's subjective well-being or the care recipient's symptoms (Sörensen et al., 2002). Thus, it would also be important to evaluate the effectiveness of psychotherapeutic interventions, which have been shown to improve these additional variables. Implementing an intervention that also improves caregiver well-being and care recipient symptoms would optimize the outcomes for caregivers and further assist them in their role.

\subsection{Cognitive Behavioural Therapy}

Most of the psychotherapy-based interventions conducted with caregivers have followed a cognitive-behavioural therapy (CBT) approach (Sörensen et al., 2002). CBT is based on the theory that thoughts, feelings, and behaviours interact, and that thoughts significantly influence our feelings and behaviours (Beck, 1975, 1993). The primary goal of CBT is to encourage participants to actively replace "irrational," "unrealistic," or "illogical" thoughts with thoughts

that are more "rational," "realistic," or "logical." The theory is that replacing unhelpful thinking styles with more realistic thought patterns will result in fewer distressing emotions and less maladaptive behaviour, which will then lead to a diminished stress response (Meichenbaum, 1996). CBT also uses relaxation exercises to try to reduce stress and emotional distress (Beck, 1975). In a meta-analysis, Pinquart and Sörensen (2006) demonstrated that CBT was related to 
improvements in burden and symptoms of depression among caregivers, but not subjective wellbeing, caregiver ability/knowledge, or symptoms of the care recipient.

For caregivers, the focus of CBT on replacing unrealistic thoughts with more realistic ones is not always helpful. Some have suggested that cognitive processes such as worrying and rumination respond less well to attempts to actively control and modify them (such as practiced in CBT) than they do to acceptance of their presence (Lundh, 2005). In contrast to CBT theory, the practice of mindfulness teaches individuals to practice acceptance toward all thoughts, behaviours, and emotions, thereby allowing them to deal with the present reality instead of ruminating about potential stress and future consequences. Whereas CBT can be viewed as an acute treatment option, mindfulness not only works in the moment, but also in a preventative way because it provides participants with a new way of thinking and behaving (Praissman, 2006). In this way, mindfulness-based stress reduction (MBSR; Kabat-Zinn, 1990) may be a more appropriate intervention for caregivers, who often face considerable unpredictability in their role. 


\section{CHAPTER FIVE}

\subsection{Defining Mindfulness}

The concept of mindfulness originates from the Buddhist tradition approximately 2500 years ago (Siegel, Germer, \& Olendzki, 2008). In ancient texts, 'mindfulness' was used to describe awareness, attention, and remembering. It was first translated into English in 1921 (Davids \& Stede, 2001). In classic mindfulness, attention is an ever-changing facet of consciousness, whereas awareness refers to a stable and specific state of consciousness; one can be constantly aware of his or her attention shifting through various sensations, thoughts, and feelings (Rapgay \& Bystrisky, 2009). In other words, attention is focused awareness (Siegel et al., 2009). The remembering aspect of mindfulness does not refer to memory of past events; rather, it refers to remembering to be aware and pay attention (Siegel et al., 2009).

The definition of mindfulness has been somewhat modified since its classical translation, and now encompasses a wide range of ideas and practices (Siegel et al., 2009). In therapeutic mindfulness literature, qualities such as nonjudgment, acceptance, and compassion have also become part of the definition. For instance, Jon Kabat-Zinn, the principle pioneer of mindfulness in Western psychotherapy, defines mindfulness as, "the awareness that emerges through paying attention on purpose, in the present moment, and nonjudgmentally to the unfolding of experience moment to moment" (Kabat-Zinn, 2003, p. 145). Germer, Siegel, and Fulton (2005) define mindfulness as, "awareness, of present experience, with acceptance" (p. 19), while Bishop et al. (2004) define it as, "self-regulation of attention so that it is maintained on immediate experience, thereby allowing for increased recognition of mental events in the present moment" (p. 232). Increased awareness is generally cultivated through formal mindfulness meditation practice. Mindfulness meditation encourages participants to choose an object of attention that is 
observable in every moment, typically the breath. Each time the mind inevitably wanders, participants are asked to pay close attention to whatever appears in the mind, from moment to moment, e.g., an itch, a sound, an emotional experience, etc. Once attention is placed on this new experience, participants practice being aware of that experience with acceptance, willingness, openness, and curiosity, while refraining from evaluating, judging, changing, or terminating it no matter how unpleasant (Baer, Walsh, \& Lykins, 2009). Upon becoming aware of the experience, attention is returned to the original object in order to re-stabilize attention. Thus, the intention of mindfulness meditation is not to choose a single object of attention and ignore other objects, but to gain insight and awareness into what arises in the mind (Siegel et al., 2009).

The process of mindfulness meditation is thought to also train cognitive functions. The cognitive mechanisms involved in mindfulness are hypothesized to be sustained attention (to maintain awareness of current experience), attention switching (to bring attention back to the present moment when it wanders), attentional inhibition (to avoid cognitive rumination) and nondirected attention (to enhance awareness of present experience, without assumptions or expectations; Bishop et al., 2004).

A number of psychologists have developed a variety of mindfulness-based interventions that aim to encourage participants to pay more attention to the present moment, while nonjudgmentally becoming aware of thoughts, feelings, and sensations (Moss, Waugh, \& Barnes, 2008). Rapgay and Bystrisky (2009) state that MBSR is the one mindfulness-based intervention that is most aligned with the perceptual and cognitive model in Buddhism, while also remaining secular and designed to suit Western mindsets and needs. They further state that many other abbreviated mindfulness interventions have lost sight of these foundations and have become "watered down versions of the original practice" (Rapgay \& Bystrisky, 2009, p. 149). 
MBSR, developed by Kabat-Zinn, was an intervention originally designed to help populations with a wide range of chronic pain and stress-related disorders (Kabat-Zinn, 1990). It is a group-based intervention that integrates mindfulness meditation and gentle Hatha yoga into a non-denominational intervention that is accessible to healthy and clinical populations with no prior meditation experience (Kabat-Zinn, 1990). Specifically, MBSR includes various sitting, walking, and eating meditations; body scan; and Hatha yoga (Kabat-Zinn, 1990). Mindful engagement in these activities is said to help participants become aware of their emotions, cognitions, and behaviours with an interested, accepting and nonjudgmental attitude (Segal, Williams, \& Teasdale, 2002).

With this new awareness, participants gain the ability to recognize their habitual thought patterns and respond to their thoughts, feelings, and behaviours in new (rather than habitual) ways. Eventually, this practice is believed to not only effect change in actual thoughts and behaviours, but also in the attitudes toward those thoughts and behaviours (Kabat-Zinn, 1990). The specific attitudes that MBSR participants learn are non-judgment, patience, beginner's mind (i.e., approaching experiences as if for the first time), trust, non-striving, accepting, and letting go (Kabat-Zinn, 1990).

Mindfulness meditation allows participants to respond to stressful internal and external stimuli in a more reflective and less reactive and impulsive way (Whitebird et al., 2013). According to the Mindful Coping Model (Garland, Gaylord, \& Park, 2009), when an experience is initially appraised as a threat that exceeds one's capabilities, individuals may initiate an adaptive response by decentering from this stress appraisal and stepping back into a mode of mindfulness. In the mindfulness mode, individuals can broaden their attention to the more expanded, metacognitive process of being aware of consciousness itself rather than remaining 
connected to its contents. This mindful state is hypothesized to increase attentional flexibility and expand awareness. From this vantage point, this theory proposes that individuals can more easily positively reappraise the distressing experience in terms of aspects that may be meaningful or beneficial (Garland et al., 2009).

This reframe is thought to give rise to more positive emotions, such as acceptance, trust, and confidence, while simultaneously decreasing ruminative thoughts about past or future events through emphasis on moment-to-moment awareness and acceptance (Borders, Earleywine, \& Jajodia, 2010; Garland et al., 2009; Jain et al., 2007). Empirical evaluation of this model has been limited, but thus far, data provide tentative support for it (Garland, Gaylord, \& Fredrickson, 2011; Garland, Hanley, Far, \& Froeliger, 2015). These skills are especially important for caregivers, who are often required to manage their own stress responses while seeing their loved one's health deteriorate, adapting to changes in their own emotions and thoughts about caregiving, and also adapting to the unpredictable nature of an individual with a neurodegenerative disease.

\subsection{Mindfulness for Non-Dementia Caregivers}

A significant number of studies suggest that increased trait mindfulness is associated with a number of positive psychological and cognitive outcomes in a variety of healthy and clinical populations, including patients with chronic pain (Kabat-Zinn, 1982), medical/premedical school students (Shapiro, Schwartz, \& Bonner, 1998), cancer outpatients (Carlson et al., 2003; Speca et al., 2000), individuals with insomnia (Gross et al., 2011), and healthy older adults (Fiocco \& Mallya, 2015). Overall, MBSR appears to be efficacious in improving psychological well-being and various stress-related health outcomes. Specifically, beneficial effects have been reported for symptoms of general distress (Astin, 1997; Carlson, Speca, Patel, \& Goodey, 2003; Speca, 
Carlson, Goodey, \& Angen, 2000), worry, rumination, and anxiety (Jain et al., 2007; Kabat-Zinn et al., 1992), depressive symptoms (Astin, 1997; Shapiro, Schwartz, \& Bonner, 1998; Speca et al., 2000), quality of life (Brown \& Ryan, 2003; Carlson et al., 2003), and cognitive function (Lenze et al., 2014). In a meta-analysis of randomized controlled trials examining the relationship between MBSR and health outcomes, Grossman, Niemann, Schmidt, and Walach (2004) calculated the mean effect size for the difference between mindfulness meditation and various control groups (e.g., relaxation, waitlist, educational material, social support plus relaxation and exercise) on a variety of mental health variables. They found a medium-sized ( $d=$ 0.54) effect, suggesting that mindfulness training might enhance the ability to cope with general distress.

To date, no studies have examined the psychological and cognitive outcomes of a standardized MBSR protocol relative to an active control group, specifically in a sample of informal caregivers for persons with dementia and related neurodegenerative diseases. However, a number of promising results have been found using various mindfulness-based interventions in other caregiving populations (see Table 1 for sample studies). 
Table 1. Studies of Mindfulness-Based Interventions for Caregivers of Adults with Medical Disorders Unrelated to Dementia

\begin{tabular}{|c|c|c|c|c|c|c|}
\hline Study & $\begin{array}{l}\text { Study } \\
\text { Design }\end{array}$ & $\begin{array}{l}\text { Care } \\
\text { Recipient's } \\
\text { Condition }\end{array}$ & $\begin{array}{l}\text { Number of } \\
\text { Caregivers }\end{array}$ & $\begin{array}{l}\text { Meditation/ } \\
\text { Comparison }\end{array}$ & $\begin{array}{l}\text { Outcome } \\
\text { Measures }\end{array}$ & Main Findings \\
\hline $\begin{array}{l}\text { Birnie et al., } \\
2009\end{array}$ & Case series & Cancer & 21 & MBSR & $\begin{array}{l}\text { C-SOSI } \\
\text { MAAS } \\
\text { POMS }\end{array}$ & $\begin{array}{l}\text { Significant improvements in mood } \\
\text { disturbance, stress, and mindfulness }\end{array}$ \\
\hline $\begin{array}{l}\text { Haines et al. } \\
(2014)\end{array}$ & Case series & $\begin{array}{l}\text { Lung } \\
\text { transplant } \\
\text { candidates/ } \\
\text { survivors }\end{array}$ & 30 & $\begin{array}{l}\text { MBSR via } \\
\text { DVD }\end{array}$ & $\begin{array}{l}\text { PSS } \\
\text { STAI }\end{array}$ & $\begin{array}{l}\text { Completers reported significantly decreased } \\
\text { perceived stress, state anxiety, and trait } \\
\text { anxiety. Non-completers did not change } \\
\text { significantly. }\end{array}$ \\
\hline $\begin{array}{l}\text { Kögler et al. } \\
(2015)\end{array}$ & RCT & $\begin{array}{l}\text { Palliative } \\
\text { patients }\end{array}$ & 57 & $\begin{array}{l}\mathrm{EBT} / \\
\mathrm{TAU}\end{array}$ & $\begin{array}{l}\text { BSI } \\
\text { CAMS-R } \\
\text { FFMQ } \\
\text { SMiLE } \\
\text { SWLS } \\
\text { WHOQOL- } \\
\text { Bref }\end{array}$ & $\begin{array}{l}\text { Mindfulness significantly associated with } \\
\text { higher quality of life, life satisfaction, the } \\
\text { experience of meaning, and lower } \\
\text { psychological distress }\end{array}$ \\
\hline
\end{tabular}

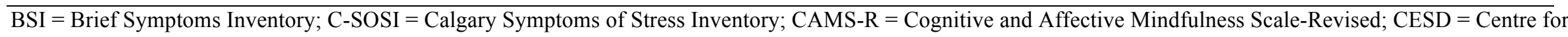
Epidemiological Studies Depression Scale; EBT = Existential Based Therapy; FFMQ = Five Facet Mindfulness Questionnaire; MAAS = Mindful Attention Awareness Scale; MBSR = Mindfulness-Based Stress Reduction; MBSR-C = Mindfulness-Based Stress Reduction for Cancer; MOS SF-36 = Medical Outcomes Studies Short-Form General Health Survey; MSAS = Memorial Symptom Assessment Scale; POMS = Profile of mood states; PSS = Perceived Stress Scale; RCT $=$ Randomized Controlled Trial; SMiLE = Schedule for Meaning in Life Evaluation; STAI = State-Trait Anxiety Inventory; SWLS = Satisfaction with Life Scale; TAU = Treatment As Usual; WHOQOL-Bref $=$ World Health Organization Quality of Life Scale (Abbreviated Version) 
Birnie, Garland, and Carlson examined the impact of an eight week, standardized MBSR program for individuals with a cancer diagnosis $(n=21)$ and their partners $(n=21)$. The program followed the Kabat-Zinn (1990) model and consisted of 8 weekly, 90-minute sessions, plus one half-day silent retreat. Participants completed the Calgary Symptoms of Stress Inventory (C-SOSI) and Profile of Mood States (POMS) questionnaires to measure perceived stress and mood, respectively. Assessments were completed within 2 weeks pre- and postintervention. Birnie et al. (2010) showed that following participation in an eight week MBSR program, caregivers of individuals with cancer reported significant reductions in symptoms of stress and total POMS score (effect sizes using Cohen's $d$ were 0.37 and 0.34 , respectively, reflecting medium-sized effects), and all POMS subscales (i.e., tension/anxiety, depression/dejection, anger/hostility, vigor, fatigue, and confusion); all $p \mathrm{~s}<.05$.

In a study conducted by Haines, Spadaro, Choi, Hoffman, and Blazeck (2014), 30 caregivers of lung transplant candidates/recipients were recruited to participate in a 4-week mindfulness-based program while their loved one was hospitalized. The mindfulness techniques were demonstrated via a DVD and each participant was asked to watch the DVD and practice the exercises for 5-15 minutes a day for four weeks. Each participant completed the Perceived Stress Scale (PSS) and State-Trait Anxiety Inventory (STAI) before and after the intervention. Results showed that PSS scores decreased significantly from pre- to post-intervention $(p=0.001)$, with a large effect size, partial $\eta^{2}=0.33$. Caregiver anxiety also showed significant improvement $(p=$ .001 ), with a large effect size, partial $\eta^{2}=0.32$.

Kögler et al. (2015) conducted a randomized controlled trial examining the effects of a mindfulness-based group intervention called Existential Behavioural Therapy in 130 informal caregivers of palliative care patients in Germany. This intervention was developed to support 
caregivers facing the imminent or recent loss of a loved one in a palliative care setting, and is defined by its emphasis on mindfulness training. Participants attended the Existential Behavioural Therapy intervention for six sessions (a total of 22 hours). The program was led by trained psychotherapists and every session included formal mindfulness practice. Participants received $\mathrm{CD}$ recordings with mindfulness exercises and were encouraged to engage in home practice at least twice a day for at least 10 minutes. Informal mindfulness practice (i.e., performing daily activities mindfully) was also encouraged. Participants completed the Cognitive and Affective Mindfulness Scale-Revised, the World Health Organization Quality of Life-BREF, and the Schedule for Meaning in Life Evaluation. The depression, anxiety, somatization, and global severity subscales of the Brief Symptom Inventory (BSI) were also administered to assess psychological distress. Questionnaires were completed immediately pre- and post-intervention, and again at 3- and 12-months follow-up.

Results demonstrated that quality of life significantly improved after the intervention, as did overall levels of depression, anxiety, and somatization on the BSI. In fact, symptoms on the BSI not only decreased significantly from pre-intervention to immediately post-intervention $(p=$ $.02, d=0.24$; small), but scores were still significantly reduced at 3-months follow up compared with pre-intervention levels ( $p=.01, d=0.25$; small). At 12-months follow-up, however, scores returned to baseline $(p=.34)$. The authors rationalized that participants may not have been practicing mindfulness frequently enough to sustain the effects. Unfortunately, the authors did not collect data on practice time at either follow-up assessment.

In a study conducted by Lengacher et al. (2012), patients who were being treated for advanced-stage breast, colon, lung, or prostate cancer $(n=26)$ and their caregivers $(n=26)$ completed a modified MBSR program (MBSR for cancer; MBSR-C) based on the Kabat-Zinn 
(1990) model. The MBSR-C program, as described in Lengacher et al. (2009), provides support for the management of specific emotional/psychological symptoms of cancer (anxiety, depression, and fear of recurrence) and physical symptoms, such as pain. The intervention has three specific components: (1) psychoeducation related to relaxation, meditation, and the mindbody connection; (2) meditation practice during group meetings and at home; and (3) group discussions and group support. The MBSR-C program was delivered via three in-person sessions and three audiotaped sessions provided on CDs. The participants completed the following measures: The PSS, the CESD, the STAI, the Memorial Symptom Assessment Scale to measure physical and psychological symptoms, and the Medical Outcomes Studies Short-Form General Health Survey to assess quality of life.

Interestingly, Lengacher et al. (2012) found that MBSR was not associated with a reduction in self-reported stress among caregivers $(p=.29, d=0.15$, small), but they did find a significant reduction in salivary cortisol after Week 1 of MBSR $(p=.005)$ and again at intervention midpoint $(p=.029)$. Moreover, they also found that after Week 1, interleukin-6 levels, a pro-inflammatory cytokine, dropped significantly $(p=.029)$. This effect may also be attributed to the change in stress perception. Quality of life showed improvement, but did not reach the threshold of statistical significance. Caregivers did not report significant reductions in depressive symptoms on the CESD upon completion of the MBSR program $(p=.30, d=0.21$, small; Lengacher et al., 2012). The authors hypothesized that this lack of change could be due to the nature of the MBSR-C program: rather than the conventional eight week duration, the MBSR-C program was reduced to 6 weeks and only 3 sessions were in-person sessions. While these modifications were intended to benefit patient participants by reducing the burden of attending eight in-person sessions, caregivers may have benefitted from the standard eight week 
protocol, as found in Birnie et al.'s (2010) study.

These studies among non-dementia caregivers have several limitations. One of the most recognizable limitations in this literature is the failure to adhere to a standard intervention protocol. Only two of the studies reviewed made use of the standardized Kabat-Zinn (1990) protocol (i.e., Birnie et al., 2010; Minor et al., 2006). The other studies restricted the number of intervention sessions (i.e., Lengacher et al., 2012), removed therapist contact completely (i.e., Haines et al., 2014), or drastically altered the intervention (i.e., Kogler et al., 2015). Although it is understandable that the standard eight week MBSR intervention may result in some burden for caregivers, it is the only standardized mindfulness-based intervention that has been rigorously evaluated for over two decades in a variety of populations, and shown to have significant effects on psychosocial well-being in otherwise healthy populations (Chiesa \& Serretti, 2009).

Only one of the reviewed studies employed an RCT design (Kögler et al., 2015), with the rest adopting a case series approach. Although case series designs are useful in determining whether an intervention is better than the passage of time, the lack of active comparison or control group in the majority of these studies makes it impossible to conclude that the changes observed were the result of program participation and not due to natural history, maturation, or regression to the mean. As such, it is difficult to discern whether factors such as expectancy or social support account for the documented benefits. RCT designs with active control groups could account for expectancy and "placebo" effects, while also helping researchers determine the "active ingredients" of mindfulness-based interventions.

\subsection{Mindfulness for Caregivers of Persons with Dementia}

The few studies that have examined the utility of mindfulness-based interventions specifically for caregivers of persons with dementia also show promising findings, but again, 
have several limitations (see Table 2 for summary of literature). For instance, Waelde, Thompson, and Gallager-Thompson (2004) conducted a pilot study with 12 older female caregivers of persons with dementia, to explore the effects of a six-session program called Inner Resources. Although this program has features similar to MBSR in some respects (e.g., use of meditation and hatha yoga), rather than using mindfulness-based meditation, it relies on a concentrative form of meditation in which practitioners focus attention on a single point, such as the breath, an image, or the silent repetition of words (mantra repetition). Results of the pre-post comparisons showed that this program is a feasible intervention for older caregivers of someone with dementia, and can improve depressive symptoms $(d=1.02)$, anxiety $(d=.68)$, and selfefficacy $(d=.66)$ with large effect sizes in this population (all $p$ s $<.05$; Waelde et al., 2004). In a more comprehensive study, Whitebird et al. (2013) conducted the first randomized control trial to investigate the effect of a standardized MBSR intervention $(n=38)$ for informal caregivers of persons with dementia relative to an active psychoeducation/support intervention ( $n$ $=40$ ). Outcomes measured included perceived stress, depression symptoms, anxiety, overall mental health, caregiver burden, and social support. Results demonstrated that MBSR was more effective than the psychoeducation/support intervention at improving overall mental health $(d=$ $0.66)$, anxiety $(d=-0.59)$, perceived stress $(d=-0.61)$, and depression $(d=-0.66)$ at 2 months post-intervention, with large effect sizes (all $p$ s $<.05$ ). 
Table 2. Studies of Mindfulness for Caregivers of Persons with Dementia

\begin{tabular}{|c|c|c|c|c|c|}
\hline Study & $\begin{array}{l}\text { Study } \\
\text { Design }\end{array}$ & $\begin{array}{l}\text { Number of } \\
\text { Caregivers }\end{array}$ & $\begin{array}{l}\text { Meditation/ } \\
\text { Comparison }\end{array}$ & $\begin{array}{l}\text { Outcome } \\
\text { Measures }\end{array}$ & Main Findings \\
\hline $\begin{array}{l}\text { Lavretsky et al. } \\
(2013)\end{array}$ & $\mathrm{RCT}$ & 39 & $\begin{array}{l}\text { KK/ } \\
\text { Relaxation }\end{array}$ & $\begin{array}{l}\text { CIRS } \\
\text { CVLT-II } \\
\text { HAMD } \\
\text { MMSE } \\
\text { SF-36 } \\
\text { TMT-A } \\
\text { TMT-B } \\
\end{array}$ & $\begin{array}{l}\text { KK significantly improved depressive symptoms, } \\
\text { mental health, and cognitive functioning compared } \\
\text { with the relaxation group. The KK group showed } \\
43 \% \text { improvement in telomerase activity compared } \\
\text { with } 3.7 \% \text { in the relaxation group }(p=0.05) \text {. }\end{array}$ \\
\hline $\begin{array}{l}\text { Waelde et al. } \\
(2004)\end{array}$ & Case Series & 12 & $\begin{array}{l}\text { Inner } \\
\text { Resources }\end{array}$ & $\begin{array}{l}\text { CESD } \\
\text { RMBPC }\end{array}$ & $\begin{array}{l}\text { Pre/post comparisons demonstrated statistically } \\
\text { significant improvements in depression, anxiety, and }\end{array}$ \\
\hline
\end{tabular}




\begin{tabular}{|c|c|c|c|c|}
\hline $\begin{array}{l}\text { Whitebird et al. RCT } \\
(2004)\end{array}$ & 78 & $\begin{array}{l}\text { MBSR/ } \\
\text { CCES }\end{array}$ & $\begin{array}{l}\text { CESD } \\
\text { MBCBS } \\
\text { MOS-SS } \\
\text { PSS } \\
\text { SF-12 }\end{array}$ & $\begin{array}{l}\text { MBSR was more effective at improving overall } \\
\text { mental health, reducing stress, and decreasing } \\
\text { depression than CCES. Both interventions improved } \\
\text { caregiver mental health and were similarly effective } \\
\text { at improving anxiety, social support, and burden. }\end{array}$ \\
\hline
\end{tabular}

ANT = Attentional Network Test; CATS = Caregiver Appraisal Tool; CCES = community caregiver education and support intervention; CESD = Centre for

Epidemiological Studies Depression Scale; CIRS = Cumulative Illness Rating Scale; CRI = Coping Responses Inventory; CRSE = Caregiving Self-Efficacy Scale; CVLT-II = California Verbal Learning Test-Second Edition; ESS = Epworth Sleepiness Scale; FFMQ = Five Facet Mindfulness Questionnaire; GPSE = General Perceived Self-Efficacy Scale; HAMD = Hamilton Depression Rating Scale; MAAS = Mindful Attention and Awareness Scale; MBCBS - Montgomery Borgatta Caregiver Burden Scale; MBSR = Mindfulness-Based Stress Reduction; MCS = Mental Health composite summary score; MMSE = Mini Mental Status Examination; MOS-SS = Medical Outcomes Study Social Support Survey; NPI = Neuropsychiatric Inventory; PSQI = Pittsburgh Sleep Quality Index; PSS = Perceived Stress Scale; PTC = Powerful Tools for Caregivers; RMBPC = Revised Memory and Behavior Problems Checklist; SCS-SF $=$ Self-Compassion Scale (Short Form); SEC = Revised Scale for Caregiving Self-Efficacy; SF-12 = Medical Outcomes Study Health Survey (Short Form); SF-36 = Medical Outcomes Study Short Form 36-Item Health Survey; STAI = State-Trait Anxiety Inventory; TMT = Trail Making Test 
Hou and colleagues (2014) recruited caregivers of persons with various chronic illnesses including hypertension, diabetes, chronic pain, and dementia, and randomly assigned them to either an eight week MBSR group $(n=70)$ or a self-help control group $(n=71)$. The MBSR intervention followed the Kabat-Zinn (1990) guidelines and consisted of eight weekly 2-hour sessions led by trained facilitators, and required participants to complete 30-45 minutes of home practice per day. The control group was required to read one chapter per week of an eightchapter self-help booklet. The booklet included information on stress acknowledgement and management, common diseases and management, skilful communication, practical home nursing advice, and advice on mental health and a healthy lifestyle. Outcome variables included depression, anxiety, perceived stress, quality of life, mindfulness, self-compassion, and confidence in obtaining respite and controlling upsetting thoughts. Participants completed these measures at baseline, post-intervention, and at 3-month follow-up.

Results showed no significant between-group differences on perceived stress scores at post-intervention $(p=.30)$ or at 3-months follow-up $(p=.62)$. The authors stated that one possible explanation for this finding was that the cultivation of mindfulness in the MBSR group changed participants' reactions to chronic perceived stress, rather than reducing the perceived stress itself, resulting in no change of perceived stress among caregivers (Hou et al., 2014).

However, Hou et al. (2014) found that caregivers randomized into an MBSR group reported significantly fewer depressive symptoms at post-intervention $(p=.002, d=-0.41$, medium) and at 3-months follow-up ( $p=.009, d=-0.36$, medium) than the self-help control group. Mindfulness training was also associated with improvement in caregiver anxiety $(d=$ 0.27 , small) and self-efficacy $(d=0.13$, small; Hou et al., 2014). Furthermore, results of this study provide important evidence that mindfulness training is related to significant reductions 
$(53 \%)$ in Traditional Chinese Medicine service utilization, and that mindfulness training may be useful in diverse populations - showing positive effects in Chinese populations (Hou et al., 2014).

Only two studies to our knowledge have evaluated the effects of mindfulness on cognitive function among older adult caregivers of persons with dementia and the results have been mixed. A pilot study conducted by Oken et al. (2010) evaluated whether a 7-week mindfulness-based cognitive therapy (MBCT) group could improve psychological, cognitive, and biological factors among middle-aged and older-adult informal caregivers of a relative with dementia. In addition to the mindfulness intervention $(n=10)$, they included two control groups: a psychoeducation group $(n=11)$ and a respite-only group $(n=10)$. Cognition was measured with a 10-word list-learning task to assess learning and memory, an abbreviated version of the Attention Network Test to measure alerting and executive control, and the Stroop Test to measure inhibition (Oken et al., 2010).

Multivariate analyses showed no group differences on either the immediate or delayed recall components of the list-learning task. Significant group differences were found on the Attention Network Test alerting score (with MBCT and psychoeducation outperforming respite), but no group differences on the executive control score. Finally, Stroop interference was significantly different between groups overall, but no post-hoc tests were reported so no conclusions can be drawn about which group was superior. Of note, examination of the means shows that the MBCT group experienced less Stroop interference than the psychoeducation and respite groups post-intervention (105.7 vs. 121.8 vs. 126.0, respectively), which Oken et al. characterize as a "suggestive effect" (p. 1036). Both intervention groups showed significant improvement on perceived stress compared with the respite group. No differences were 
demonstrated between groups on any biological measures. Although this pilot study was rigorous in terms of its two control arms, future studies must be conducted with a larger sample size and with more specific analyses.

Lavretsky et al. (2013) examined the effects of an eight week daily yogic meditation intervention on psychological, cognitive, and biological factors among elderly caregivers of someone with dementia. Caregivers were randomized to either daily Kirtan Kriya (KK; i.e., a 12minute yogic meditation chanting practice; $n=23$ ) or Relaxing Music (i.e., listening to relaxing instrumental music for 12 minutes; $n=16$ ) conditions. The KK group showed clinically significant improvement on measures of global cognitive function $(d=1.12)$ and executive function (i.e., set shifting; $d=.82$ ), and greater improvement in self-reported overall mental health $(d=.64)$ with large effect sizes, and significant reductions in depressive symptoms $(d=$ .51 , medium), all $p \mathrm{~s}<.05$. Moreover, the KK group showed significant improvement in telomerase activity, signifying improvement in stress-induced cellular aging $(d=.56$, medium; Lavretsky et al., 2013).

Although both of these studies are methodologically sound in terms of using a controlled study design, the sample sizes were quite small. Moreover, neither study reported on participant attendance or frequency of home practice, which help researchers understand the dose-response relationship of mindfulness-based intervention. Neither study applied a standardized, evidencebased intervention, which reduces the ability to generalize their results. Lastly, neither study used a comprehensive battery of cognitive measures that is sensitive to the range of stress-related cognitive declines caregivers have been shown to experience (i.e., global cognitive function, verbal ability, processing speed, attention, episodic memory, and executive function). In light of these mixed findings and limitations, the current study proposed to assess whether, relative to an 
active control group, a standardized MBSR intervention can improve performance on a comprehensive battery of cognitive and psychosocial measures, in a sample of older caregivers of individuals with a neurodegenerative disease.

\subsection{Objectives and Hypotheses}

Given the documented robust association between caregiving, stress, and cognitive decline, the goal of this dissertation is to address the following objectives and hypotheses:

Objective I: To determine whether caregivers show significant differences in performance on measures of cognitive and psychosocial function relative to healthy, community sample of older adults.

- Hypothesis 1: Relative to the community sample, caregivers will show decreased performance on measures of cognitive flexibility (as measured by the TMT-B), attention (TMT-A), verbal learning and delayed recall (as measured by the CVLT-II), verbal fluency (as measured by the Controlled Oral Word Association Task; COWAT), and significantly poorer outcomes on measures of perceived stress, mindfulness, quality of life, mood, and self-esteem.

Objective II: To determine whether MBSR is associated with significant change in cognitive function relative to the control group.

- Hypothesis 2: The primary hypothesis is that MBSR will produce significantly greater pre-to-post intervention improvements in cognitive flexibility than a psychoeducational control intervention. TMT-B completion time will serve as the primary measure to test this hypothesis. Supplementary measures of cognitive function will include the TMT-A, CVLT-II, Digit Span, WAIS-IV Coding and Symbol Search, COWAT, and Stroop Task. Objective III: To determine whether MBSR is associated with significant change on measures of 
psychosocial functioning relative to the control group.

- Hypothesis 3: MBSR will produce significantly greater pre-to-post intervention improvements than the control group on the following psychosocial measures: the Five Facet Mindfulness Questionnaire (FFMQ; Baer, et al., 2006) the Perceived Stress Scale (PSS; Cohen, Kamarck, \& Mermelstein, 1983), the Centre for Epidemiological Studies Depression Scale (CESD; Radloff, 1977) the Rosenberg Self-Esteem Scale (RSES; Rosenberg, 1965), the Quality of Life Scale (QOLS; Flanagan, 1978), and the Burden Interview (Zarit, Reever, \& Bach-Peterson, 1980). 


\section{CHAPTER SIX}

\section{Method}

In order to examine the stated study objectives, two sample cohorts were recruited and tested (i.e., a community sample of older adults and a sample of caregivers). These two cohorts allowed for the assessment of caregiving status on cognitive and psychosocial function.

\subsection{Cohort 1: Community Sample}

\subsubsection{Participants \& Recruitment}

A previous study by de Vugt et al. (2006) found that caregivers showed significantly decreased performance on a measure of delayed verbal recall than non-caregivers. In order to detect a similar effect, a power analysis using a formula derived from Crawley (2005) determined that a sample size of 108 participants (54 per group) was required. A total of 97 healthy, communitydwelling older adults between the ages of 63 and $81(M=69.16, S D=4.84)$ were recruited between 2013-2015 for a previous study (see Mallya \& Fiocco, 2015).

Participants were recruited through a combination of online recruitment advertisements, educational community talks/events, and through the Ryerson Senior Participant Pool, which is a Ryerson University-maintained database of older adult participants. Furthermore, they were required to have English-language proficiency, and have normal or corrected-to-normal hearing and vision. Participants were excluded if they endorsed any medical or psychiatric conditions that might significantly affect cognitive performance, such as a neurodegenerative disorder, recently diagnosed psychopathology, stroke, or diabetes. Participants were also excluded if they endorsed regular participation in any mindfulness or meditation-based activities such as yoga or tai chi. Lastly, individuals were excluded if they had received cognitive testing in the past year using a similar cognitive battery due to potential learning effects. 


\subsubsection{General Procedure}

This study protocol was approved by the Ryerson University Research Ethics Board (REB 2012192). Eligible participants were told that they would be randomized into either an eight week MBSR group or a progressive muscle relaxation group after completion of a 1-hour testing session that occurred one to two weeks prior to commencement of the intervention. Following consent, participants completed a battery of cognitive tests and a series of psychological questionnaires, including the TMT-B, CVLT-II, COWAT, MMSE, QOLS, RSES, and Geriatric Depression Scale (GDS). After the battery was complete, the participants were told whether they were assigned to MBSR or the relaxation control group. After the eight week intervention phase, all participants were asked to meet with the same research assistant at the same time of day and complete a post-intervention testing session in which a comparable battery of cognitive tests was administered (See Mallya \& Fiocco [2015] for full study description). For the present study, baseline performance on the TMT-B, CVLT-II, COWAT, MMSE, QOLS, RSES, and GDS was used to address the first study objective.

\subsection{Cohort 2: Caregivers}

\subsubsection{Participants \& Recruitment}

A previous study by Lavretsky et al. (2014) found a large effect size $(d=0.82)$ when comparing caregivers' change scores in outcomes on the TMT-B following a meditation or relaxation group. For the main analysis, in order to detect a similarly large effect size in this sample with power of .80 and an alpha of .05 for a Mixed ANOVA, an a priori power analysis using G-Power determined that a sample size of 36 caregivers (18 per group) was required. To account for an approximate $20 \%$ attrition rate, a total of 44 caregivers (22 per group) aged $50+$ were required to be randomized into the study. 
Participants were recruited through a partnership with the Mississauga-Halton Local Health Integration Network (LHIN), a combination of online and print advertisements, as well as educational community talks/events. Individuals who responded to the study advertisement were provided with details of the study purpose and procedure during a telephone call (Appendix A), at which point they were screened for eligibility.

Eligible individuals were required to be at least 50 years of age and living independently in the community. They were required to be caregivers for a relative or spouse with an agerelated neurodegenerative disease. Previous research has suggested that there is no correlation between the subtype of neurodegenerative disease and the degree of self-reported caregiver burden (Tang et al., 2013) or distress (Habermann \& Davis, 2005), and thus, caregivers of any subtype of age-related neurodegenerative disease were included. Previous research has also demonstrated that informal caregivers who place their loved one in a long-term care facility experience comparable levels of depression, anxiety, and burden to caregivers who care for their loved one at home, with causes of burden including travel for visiting, making decisions about activities that might interfere with visiting, difficulties encountered with staff, and financial concerns (Baumgarten et al., 1994; Stephen, Ogrocki, \& Kinney, 1991). Thus, caregivers who reported that their loved one was institutionalized, but still reported significant involvement with their care, were included.

All eligible participants were required to have English-language proficiency, have normal or corrected-to-normal hearing and vision, and be able to attend eight weekly sessions at one of the offered locations. Participants were excluded if they endorsed any medical or psychiatric conditions that might significantly affect cognitive performance, such as a neurodegenerative disorder, stroke, or uncontrolled diabetes. Participants who endorsed regular, active participation 
in any mindfulness-based activities such as yoga, meditation, or tai chi were also excluded.

Further, individuals were excluded if they had undergone neuropsychological testing in the past year using a similar cognitive battery due to potential learning effects (see Appendix B for full screening criteria). Participant eligibility was assessed by Sasha Mallya and trained research assistants associated with the study.

\subsubsection{Design}

Enrolled participants were asked to undergo a one-hour pre-intervention testing session one to two weeks prior to commencement of the intervention. The session required participants to complete a series of cognitive measures and self-report questionnaires. After the battery was complete, the participant was told whether they were assigned to the MBSR condition or the psychoeducation condition. Testing sessions and interventions were offered in the Greater Toronto Area (Oakville and Etobicoke) or in the City of Toronto (North York and downtown). Participants could choose their preferred location in order to minimize potential burden to attend the group.

With regard to randomization, strict randomization was not feasible due to caregivers' time constraints and scheduling conflicts. All efforts were made to hold the intervention and control groups concurrently, each taking place during the same eight week period, at the same time of day on different days (e.g., the intervention group on Tuesdays at $10 \mathrm{AM}$ and the control group on Thursdays at $10 \mathrm{AM}$ over the same eight week period). At the time of screening, eligible participants were informed of the two weekdays when the interventions would be taking place (e.g., Tuesdays or Thursdays) but were not informed as to which intervention would be held on each day. If the eligible participant indicated that he/she was available on both weekdays for eight weeks, group allocation was based on randomization using a list randomizer. However, 
for logistical reasons, participants who were only available on one of the two days were allocated to the group that they could attend. It is important to emphasize that although participants could choose their preferred location and time, they were not aware of the group that they would be allocated to until the end of their first testing session.

The testing process was administered once again one to two weeks after the intervention was completed. All participants were required to sign a consent form prior to study commencement (see Appendix C) and were debriefed after all study procedures were complete (see Appendix D). This study protocol was approved by the Ryerson University Research Ethics Board (REB 2014-118).

\subsection{Interventions}

The two groups offered in Study 2 consisted of an MBSR intervention and an active psychoeducation group intervention adapted from the psychoeducation interventions described earlier. Each intervention ran for an eight week time period given that this is the typical duration for the MBSR protocol. Moreover, it is a length of time during which we would expect little change in the care recipient's condition and reasonably high group attendance by the caregivers. A 2-hour session length was chosen. Although this duration is slightly less than the usual length of MBSR interventions (2.5 hours), it was considered the best compromise to maximize class attendance since caregivers may experience significant burden if asked to part with their care recipient for much longer. Participants in the MBSR group were also offered an optional halfday silent session, as per standard MBSR protocol.

Sasha Mallya facilitated three psychoeducation groups and two MBSR groups. Dr. Alexandra J. Fiocco facilitated three MBSR groups. Both investigators received mindfulnessbased facilitator training through the Sunnybrook Health Sciences Centre. A facilitator feedback 
questionnaire adapted from Stirman et al. (2013) was administered to participants at the end of each intervention to ensure that facilitators were consistent across groups, and comparable to each other on a variety of non-specific characteristics. Participants who dropped out of the intervention were contacted in order to document their reason(s) for discontinuing their participation. Participants were enrolled in groups to one of the following interventions:

\subsubsection{Mindfulness-based stress reduction (MBSR)}

The MBSR intervention followed the guidelines created by Kabat-Zinn (1990). The intervention consisted of 8 weekly group sessions lasting 2 hours each, with an optional half-day session between week 6 and 7. Every week, group members were presented with a topic of discussion and learned mindfulness-based techniques to help them manage stress. As per Kabat-Zinn's recommendations, the weekly discussion topics included: Automatic Pilot; Mindfulness of the Body and Reacting vs. Responding; Mindfulness of the Breath; Mindfulness and Physiological Stress Reduction; Self-Acceptance, Allowing, and Letting Be; Interpersonal Relationships; Integrating Self-Care and Mindful Eating; and Mindfulness for the Rest of Your Life. Each week, participants were taught various meditation practices such as body scans, light hatha yoga, mindful walking, and various sitting meditations that were applied during the group and at home. Informal mindfulness practices such as mindful eating and mindful participation in routine activities (e.g., doing the dishes) were also encouraged, to allow participants to cultivate mindfulness beyond the formal practices. Participants were asked to complete approximately 30 minutes of homework per day, which consisted of practicing the techniques learned in each week's group.

\subsubsection{Psychoeducation Intervention}

The psychoeducation control group also consisted of eight weekly group sessions lasting 2 hours 
each. The program was primarily characterized by the provision of psychoeducational information. Over the course of the program, participants learned about a number of resources and tools to help them manage their caregiving responsibilities. The weekly topics included: Understanding Dementia; Caregiver Burnout; Accessing Services; Effective Communication; Life Balance; Nutrition; Fitness; and Rewards of Caregiving. Not only did participants learn new information provided by the facilitator, but they also had a chance to ask questions, discuss current issues, and gain social support from their caregiver peers. Participants were not taught any mind-body techniques (e.g., cognitive-behavioural strategies, meditation, relaxation, visual imagery, yoga, etc.).

\subsection{Measures}

\subsubsection{Cognitive Measures}

Global cognitive function. The Mini-Mental State Examination (MMSE; Folstein et al., 1975) is one of the most widely used brief screening instruments for global cognitive impairment. It is also used to track cognitive changes over time and to assess the effects of psychotherapeutic interventions on cognitive function (Strauss, Sherman, \& Spreen, 2006). The items assess orientation to time and place, attention and calculation, language, and immediate and delayed recall. It provides a total score ranging from 0 to 30 , with lower scores indicative of greater cognitive impairment. Research has demonstrated that a cut-off score of 27 (i.e., 26 or below) results in an optimal balance of sensitivity and specificity (.89 and .91, respectively) with an overall correct dementia classification rate of 90\% (O’Bryant et al., 2008).

Executive Function (Set Shifting) The Trail Making Tests A and B (TMT-A and TMTB; Reitan \& Wolfson 1993) measure a number of different functions, including information processing speed, visual scanning ability, complex attention, integration of visual and motor 
functions, and set shifting (Crowe, 1998). TMT-A requires participants to connect 25 consecutively numbered circles using straight lines. TMT-B requires subjects to connect consecutive numbers and letters, alternating between the two sequences (i.e., 1-A-2-B-3-C). The TMT is a timed task, and faster completion time (along with fewer errors) is associated with better functioning. Performance on the TMT-A is thought to be more uniquely indicative of psychomotor speed, while TMT-B completion time is more related to set shifting (Crowe, 1998). TMT-B errors are associated with deficits in inhibition (Amieva et al., 1998). TMT-B is sensitive to age-related decline in executive function (Rasmusson et al. 1998). Previous research indicates that TMT scores are sensitive to change after an MBSR intervention (Johansson et al., 2012).

An alternate version of the TMT was administered and counterbalanced to minimize learning effects. The alternate version of the TMT is comparable to the original version; Franzen et al. (1996) found no significant differences in overall level of performance between TMT-A and its alternate form, and between TMT-B and its alternate form, in a sample of closed head injury patients. These authors found reliability estimates of $r=0.70$ for TMT-A and its alternate form and $\mathrm{r}=0.78$ for TMT-B and its alternate form. Their results indicate adequate reliability of the alternate forms for TMT. TMT-B completion time was used as the primary outcome variable, as studies have shown that this outcome variable is one of the strongest neuropsychological predictors of functional independence (Hanks et al., 2008; Marshall et al., 2011).

Executive Function (Updating) The Controlled Oral Word Association Task (COWAT; Benton, 1989) was used to measure verbal fluency. The COWAT consists of two tasks: phonemic fluency and semantic fluency. Participants are given 1 minute to verbally generate as many words as they can think of when prompted with a letter of the alphabet (F, A, and S; phonemic fluency), followed by a category (Animals; semantic fluency). Both components 
measure the ability to access semantic memory and draw on executive processes. Specifically, participants use these abilities to retrieve words within a given phoneme or category and keep track of words that have already been produced to avoid repetition (Shao, Janse, Visser, \& Meyer, 2014). Phonemic fluency has been found to be more dependent on frontal lobe functioning, whereas semantic fluency is more sensitive to temporal lobe functioning (Henry \& Crawford, 2005). This measure is sensitive to age-related decline in verbal fluency (Steinberg et al. 2005) and has been used previously with older adults (Anderson-Hanley et al. 2012). The alternate version of the COWAT was not used, as previous research indicates a difference in difficulty between the alternate and original forms (Barry et al. 2008). Performance on this measure is sensitive to change after a mindfulness-based intervention (Zeidan, Johnson, Diamond, David, \& Goolkasian, 2010).

Executive Function (Inhibition) The Stroop Color and Word Test (Golden, 1978) requires deliberate inhibition of an automatic response. First, participants are required to name the colour of printed words that are congruent with the meaning of the word (e.g., the word "red" printed in red ink). Response time on this component is compared to response time when the colour of the word is incongruent to its meaning (e.g., the word "red" printed in blue ink). The Stroop interference effect is the finding that participants are faster at the congruent condition than the incongruent condition. Higher levels of mindfulness are associated with reduced interference (Moore \& Malinowski, 2009).

Episodic Verbal Learning and Memory The California Verbal Learning Test - Second Edition (CVLT-II; Delis et al., 2000) measures verbal learning, immediate and delayed recall, and recognition for two lists of words: a primary List A and a secondary interference List B. Each list consists of 16 words; four words from four semantic categories. The participant is 
presented with five learning trials of List A, followed by the interference List B, and then shortdelay free- and cued-recall of List A. After a 20-minute delay, the participant completes longdelay free-recall, cued-recall and yes/no recognition trials of List A. The CVLT-II provides numerous parameters of learning and memory and for this study, we analyzed total number of words learned across five trials (verbal learning) long delay free recall of List A (verbal memory), and frequency of repetition errors in recall (monitoring). We administered the alternative form for the second testing session to control for practice effects (Delis et al., 1991). The CVLT-II has been used to examine learning and memory in older adults (Richmond, Morrison, Chein \& Olson, 2011) and is sensitive to change after a meditation-based intervention (Lavretsky et al., 2011).

Attention \& Working Memory The WAIS-IV Digit Span (DS) subtest requires the participant to reproduce progressively longer lists of digits that are read out loud by the test administrator at a rate of approximately one digit per second (Wechsler, 2008). The task consists of three conditions: Digits Forward (DSF), Digits Backward (DSB), and Digits Sequencing (DSS). The DSF task requires participants to repeat a sequence of random digits between 1 and 9 (inclusive) in forward order, beginning with a 2-digit sequence. Two items are presented for each sequence length, and if the participant reproduces at least one item correctly, the sequence length is increased by one until the participant repeats both items incorrectly at the same sequence length, up to a maximum of a 9-digit sequence. The DSB task is identical to the DSF task except that participants must reproduce the sequences in reverse order. The DSS task requires participants to repeat the sequence in ascending numerical order. DSF is sensitive to attention, while DSB and DSS are more sensitive to working memory (Lichtenberger \& Kaufman, 2013). Performance on DS is sensitive to change after a mindfulness-based intervention (Chambers et 
al., 2008; Zeidan, et al., 2010).

Processing Speed The Coding and Symbol Search tasks are subtests from the WAIS-IV (Wechsler, 2008). Both measure processing speed. In the Coding subtest, participants are shown a series of symbols that are paired with numbers. With the help of a legend, participants must draw the correct symbol under its corresponding number, within a 120 second time limit. For the Symbol Search subtest, participants visually scan two groups of symbols (a target group and a search group) and indicate if either of the target symbols matches any of the symbols in the search group. The participants respond to as many items as possible within a 120 second time limit. The primary outcome variable for these tests is the total number of correct responses. Coding is more demanding of fine-motor skills, short-term memory, and learning ability, and Symbol Search is more demanding of attention to detail and visual discrimination. Together, they form a Processing Speed Index score. Performance on the Coding task has been found to improve after completion of an MBSR intervention (Johansson, Bjuhr, \& Rönnbäck, 2012).

\subsubsection{Psychosocial Measures}

Mindfulness The Five Facet Mindfulness Questionnaire (FFMQ) is a 39-item questionnaire that measures responses on a 5-point Likert scale, ranging from 1 "never or very rarely true" to 5 "very often or always true". It is considered to be the most comprehensive measure of mindfulness as it integrates common factors from several other mindfulness questionnaires. The five factors include: 1) Observing (e.g., "When I'm walking, I deliberately notice the sensations of my body moving"); 2) Describing (e.g., "I'm good at finding words to describe my feelings"); 3) Acting with Awareness (e.g., "When I do things, my mind wanders off and I'm easily distracted"); 4) Non-judging (e.g., "I criticize myself for having irrational or inappropriate emotions"); and 5) Non-reacting (e.g., "I perceive my feelings and emotions 
without having to react to them"). Responses are summed and higher scores indicate greater levels of mindfulness. Each of the five factors show high internal consistency: Non-reactivity $\alpha=$ .75 , Observing $\alpha=.83$, Acting with Awareness $\alpha=.87$, Non-judging $\alpha=.87$, and Describing $\alpha=$ 91. Construct validity and psychometric properties have been tested in various samples (Baer et al., 2006).

Burden The Burden Interview (Zarit, Reever, \& Bach-Peterson, 1980) is one of the most widely used scales to measure caregiver burden. It consists of 22 items that measure responses on a 5-point Likert Scale (from 0 "never" to 4 "nearly always"). Items were derived from clinical and research experience with caregivers of persons with dementia and show high internal consistency $(\alpha=.91)$ and test-retest reliability $(\alpha=.71$; Zarit et al., 1980). The Burden Interview is scored by adding the numbered responses of the individual items, with higher scores indicating greater caregiver burden. Degree of burden is interpreted through the use of four cut-off points: $\leq$ 20 "Little or no burden"; 21-40 "Mild to moderate burden"; 41-60 "Moderate to severe burden"; and 61-88 "Severe burden".

Perceived Stress The Perceived Stress Scale (PSS; Cohen et al., 1983) measures the degree to which situations in one's life are appraised as stressful. The scale includes items designed to measure how often individuals find their lives unpredictable, uncontrollable, and overloaded during the last month. Internal consistency is good $(\alpha=0.85)$, and the stability of the test-retest coefficients ranges from 0.75 to 0.86 (Cohen et al., 1983). This scale has been used among older adult populations (Hamarat et al., 2001) and has been shown to be sensitive to change with a MBSR intervention (Shapiro et al., 2007).

Quality of Life The Quality of Life Scale (QOLS; Flanagan, 1978) measures satisfaction with needs met. It contains 16 items representing six domains of quality of life: physical and 
material well-being, relationships with other people, social and civic activities, personal development, and independence. Cronbach's alpha coefficient has been documented at 0.87 for the general adult population (Burckhardt et al., 1989). This scale has demonstrated sensitivity to change following an MBSR intervention (Azulay et al., 2012).

Self-esteem The Rosenberg Self-Esteem Scale (RSES; Rosenberg, 1965) is a ten-item measure that assesses global self-esteem. The RSES contains an equal number of positively and negatively worded items and responses are coded on a four-point scale. It is considered to be an appropriate measure of self-esteem in the elderly (Breytspraak \& George, 1979) and a powerful predictor of increased reactivity to stress (Pruessner et al., 2005). This scale has previously demonstrated sensitivity to change following an MBSR intervention (Goldin \& Gross, 2010).

Depression The CES-D (Radloff, 1997) is a well-validated, 20-item, scale that measures depressive symptomatology. Participants are asked to rate statements based on their symptoms over the last week, such as "I felt happy" and "I felt depressed" on a four point Likert scale (03) and scores range from 0 to 60 with higher scores indicating higher levels of depression. The CES-D also provides cutoff scores (i.e., 16 or greater) that aid in identifying individuals at risk for clinical depression, with good sensitivity and specificity and high internal consistency (Lewinsohn, Seeley, Roberts, \& Allen, 1997). The CES-D has been used successfully in older adults (Lewinsohn et al., 1997), is sensitive to differences between caregivers and non-caregivers (Pinquart \& Sorensen, 2003), and is sensitive to changes in depressive symptoms after a mindfulness intervention (Zeidan et al., 2010).

Adherence Attendance was taken at each group meeting. In order to be considered adherent to treatment, participants needed to attend at least six of eight sessions (see Appendix E). Participants in the MBSR group completed a weekly paper log to record which practice they 
completed each day, for how many times, and for how many minutes (see Appendix F).

Feedback Questionnaire At the end of the follow-up testing session, all participants were asked to complete a feedback questionnaire, consisting of a facilitator component and an intervention component (see Appendix G). The facilitator feedback component instructed participants to rate their group facilitator on a variety of domains in order to determine whether the facilitators of each intervention were comparable in terms of therapist effects (adapted from the Cognitive Processing Therapy: Therapist Adherence and Competence protocol; Stirman et al., 2013). The intervention feedback component asked participants to describe their thoughts about the program (e.g., whether they enjoyed it, which aspects they found helpful, if they could recommend any changes, etc.).

\subsection{Statistical Analyses}

All outcome data were first assessed for normality and homogeneity of variance and met these assumptions. Using the Outlier Labeling Rule (Hoaglin et al. 1986), outliers were discovered. In the community sample of older adults, six outliers were found: three participants had slower TMT-B completion time, two participants showed higher scores on the GDS, and one participant showed lower quality of life. Within the sample of caregivers, four participants had significantly more repetitions on the CVLT-II, one participant scored significantly lower on Digit Span Forward, three participants scored significantly higher on Digit Span Backward, three participants were significantly slower to complete TMT-A, and one participant made significantly more errors on the TMT-B. There were no outliers for psychosocial variables. Data for both samples were analyzed with and without outliers and given that exclusion of outliers did not significantly change statistical outcomes, all reported analyses contain the outlier data points.

To test the first hypothesis that caregivers would show decreased performance on 
measures of cognitive and psychosocial function relative to the community sample of older adults, one-way analysis of covariance (ANCOVAs) were performed. Demographic variables that significantly differed $(p<.05)$ between groups at baseline were considered covariates. Age, sex, and education were used as a priori covariates in all analyses, as these factors are all associated with performance on measures of cognitive function (van Hooren et al., 2007). To test the hypothesis that MBSR would produce significantly greater pre-to-post intervention improvements on measures of cognitive and psychosocial function relative to the psychoeducation group, one-way analysis of variance (ANOVA) and $\chi^{2}$ analyses were first used to analyze between-group differences at baseline. Variables that significantly differed $(p<.05)$ between groups at baseline were considered covariates. A priori covariates (sex, education, and age) were entered in all subsequent analyses (Brayne \& Calloway 1990; Deary et al., 2009; Tombaugh, 2004). Then, a mixed $2 \times 2$ ANCOVA was performed, using pre-intervention and post-intervention scores as the within groups factor, and group assignment (MBSR, Psychoeducation) as the between groups factor. Separate analyses were conducted on all participants who were randomized into the study regardless of whether they completed the intervention or not (i.e., intention to treat [ITT] analyses), and on the subsample of participants that completed at least $75 \%$ of the sessions (i.e., per protocol [PPT] or completer analyses). All analyses were considered significant at $p<0.05$.

Although TMT-B completion time was the primary outcome variable, between-groups differences were analyzed for all cognitive and psychosocial variables. Comparing multiple variables in the same sample may increase the risk of a Type I error. The sample size was too small to support Bonferroni corrections, so no statistical correction was made for multiple comparisons and instead, all of the individual $p$ values and effect sizes are reported for each 
comparison (Rothman, 1990). 


\section{CHAPTER SEVEN}

\section{Results}

\subsection{Study 1: Caregivers versus Community Sample}

Baseline characteristics of the two groups are shown in Table 3. The caregivers and the community sample did not differ in sex distribution or level of education, but the caregivers were significantly younger.

Contrary to the stated hypotheses, ANCOVAs suggested no statistically significant differences between groups on CVLT-II LDFR or TMT-B completion time, although examination of TMT-B means showed a trend in the expected direction. With regard to supplementary measures of executive function, several significant differences were found: caregivers made more errors on the TMT-B, generated fewer words on both components of the COWAT (FAS and Animals), and learned significantly fewer words on the CVLT-II. Marginally significant differences were found in time to complete TMT-A. In terms of psychosocial variables, caregivers reported significantly more perceived stress and depressive symptoms, and reported lower quality of life. On average, caregivers also reported being less mindful overall (see Table 3).

Table 3. Community sample compared with caregivers, controlling for age, sex, and education

\begin{tabular}{|c|c|c|c|c|c|}
\hline Variable & $\begin{array}{l}\text { Community } \\
\text { Sample }(n= \\
97) \\
\text { Mean (SD) }\end{array}$ & $\begin{array}{l}\text { Caregivers }(n= \\
57) \\
\text { Mean (SD) }\end{array}$ & $F(1,151)$ & $p$ & Partial $\eta^{2}$ \\
\hline Age, years & $69.16(4.84)$ & $66.26(7.55)$ & 8.35 & $.004 * *$ & .052 \\
\hline $\begin{array}{l}\text { Sex, N (\% } \\
\text { female) }\end{array}$ & $71(72 \%)$ & $46(81 \%)$ & $\chi^{2}(1)=1.33$ & .25 & $\Phi=.093$ \\
\hline Education, years & $16.20(3.36)$ & $16.39(2.94)$ & .114 & .74 & .001 \\
\hline MMSE & $28.78(1.68)$ & $28.64(1.46)$ & .937 & .35 & .007 \\
\hline $\begin{array}{l}\text { CVLT, Total } \\
\text { Words }\end{array}$ & $50.56(11.98)$ & $47.63(10.56)$ & 7.054 & $.009 * *$ & .047 \\
\hline CVLT, LDFR & $10.90(3.60)$ & $10.70(2.80)$ & 1.068 & .30 & .007 \\
\hline
\end{tabular}




$\begin{array}{llllll}\text { CVLT, } & 5.79(7.03) & 5.25(6.56) & .138 & .71 & .001 \\ \text { Repetitions } & & & & \\ \text { TMT-A, time (s) } & 35.18(11.02) & 39.39(21.81) & 3.508 & .06 & .024 \\ \text { TMT-B, time (s) } & 82.96(37.55) & 87.43(34.50) & 2.938 & .09 & .020 \\ \text { TMT-B, errors } & .50(.834) & .71(.97) & 5.39 & .02^{*} & .037 \\ \text { TMT B-A/A } & 2.46(1.17) & 2.39(.70) & .036 & .85 & .000 \\ \text { COWAT - FAS } & 46.71(11.65) & 41.84(12.50) & 7.580 & .007^{* *} & .050 \\ \text { COWAT - } & 20.52(5.29) & 18.04(5.12) & 13.138 & .000^{* * *} & .084 \\ \text { Animals } & & & & & \\ & & & & & \\ \text { Depressed } & 19 \% & 60 \% & \chi^{2}(1)=26.60 & .000^{* * *} & \Phi=.420 \\ \text { RSES } & 24.00(4.93) & 22.71(5.22) & 2.866 & .09 & .020 \\ \text { FFMQ" } & & & & & \\ \quad \text { Observe } & 28.36(5.60) & 25.76(5.60) & 3.428 & .07 & .047 \\ \quad \text { Describe } & 31.18(6.60) & 28.04(5.48) & 3.788 & .06 & .051 \\ \text { Awareness } & 29.95(5.18) & 25.71(6.90) & 6.400 & .01^{*} & .084 \\ \quad \text { Nonjudgment } & 31.00(5.85) & 26.15(6.38) & 11.638 & .001^{* *} & .143 \\ \quad \text { Nonreactive } & 24.14(4.14) & 20.91(4.40) & 6.198 & .02^{*} & .081 \\ \text { PSS } & 13.78(6.79) & 22.45(6.64) & 52.376 & .000^{* * *} & .271 \\ \text { QOLS } & 86.89(12.50) & 72.49(13.82) & 39.902 & .000^{* * *} & .221\end{array}$

${ }^{*} p<.05, * * p<.01, * * * p<.001$

${ }^{\S}$ Note: In the community sample, the GDS was used to assess depressive symptoms, while in the caregiving sample the CES-D was used. As such, the percentage of participants endorsing clinically significant levels of depressive symptomatology is reported.

"Note: In the community sample, the FFMQ was added later than other measures; 22

participants completed this measure.

MMSE $=$ Mini-Mental State Examination, CVLT $=$ California Verbal Learning Test-II, LDFR $=$ CVLT Long Delay Free Recall, TMT $=$ Trail Making Test, COWAT $=$ Controlled Oral Word Association Task, RSES = Rosenberg Self-Esteem Scale, FFMQ = Five Facet Mindfulness Questionnaire, PSS $=$ Perceived Stress Scale, QOLS $=$ Quality of Life Scale

\subsection{Study 2: Randomized Controlled Trial for Caregivers}

\subsubsection{Recruitment and Study Flow}

As shown in the CONSORT flow diagram of recruitment (Figure 2), a total of 196 caregivers showed interest in this study. We excluded 139 potential participants and 57 eligible participants were randomized into one of the two groups. Regarding study attrition, 43 participants (75\%) completed the study (i.e., attended at least 6 out of 8 sessions). There were $23(70 \%)$ completers in MBSR and 20 (83\%) in the Psychoeducation group. Although there were more noncompleters in the MBSR group, this difference was not significant, $\chi^{2}(1)=1.39, p=.24$. All 57 
participants were included in the ITT analyses, while 43 participants were included in PPT analyses.

Of the 10 participants who did not complete the MBSR program, five participants stated that time constraints limited their ability to attend; one person decided that she already had sufficient coping skills; and four people did not respond. Of the four people who did not complete the Psychoeducation group, one person stated that he no longer had someone who could care for his care recipient while he attended the group; two people had to attend too many appointments that coincided with session times; and one person completed baseline assessment but did not attend any intervention sessions due to unforeseen scheduling conflicts.

One-way ANOVAs were run to test for differences in demographic factors and dependent variables that may have differentiated completers and non-completers. There were no demographic differences, but dropouts were significantly slower to complete TMT-A $(F(1,55)=$ $11.51, p=.001)$ and TMT-B $(F(1,54)=4.46, p=.04)$. Of the 57 individuals randomized into the study, $50(88 \%)$ attended the post-intervention follow-up cognitive testing session. Both group facilitators were rated equally on all outcome variables (i.e., genuineness, warmth, empathy, engagement, efficiency, ability to provide feedback, providing clear homework instructions, and problem-solving), all $p s>.05$. On average, participants reported completing 112.32 (72.14) minutes of home practice per week, although the most commonly reported number of minutes spent in home practice was 0 minutes.

\subsubsection{Baseline Characteristics of the Sample}

Baseline characteristics of the caregivers across intervention group are shown in Table 4. The MBSR and control group did not significantly differ in sex distribution, level of education, or any of the psychosocial variables. However, participants in the MBSR group were significantly 
younger and recalled significantly more words across CVLT-II learning trials than participants in the Psychoeducation group. An analysis was run comparing groups on CVLT-II LDFR controlling for total words learned, and no significant result was found, thus, we did not assess this further.

Figure 2. CONSORT flow diagram of randomized controlled trial for caregivers

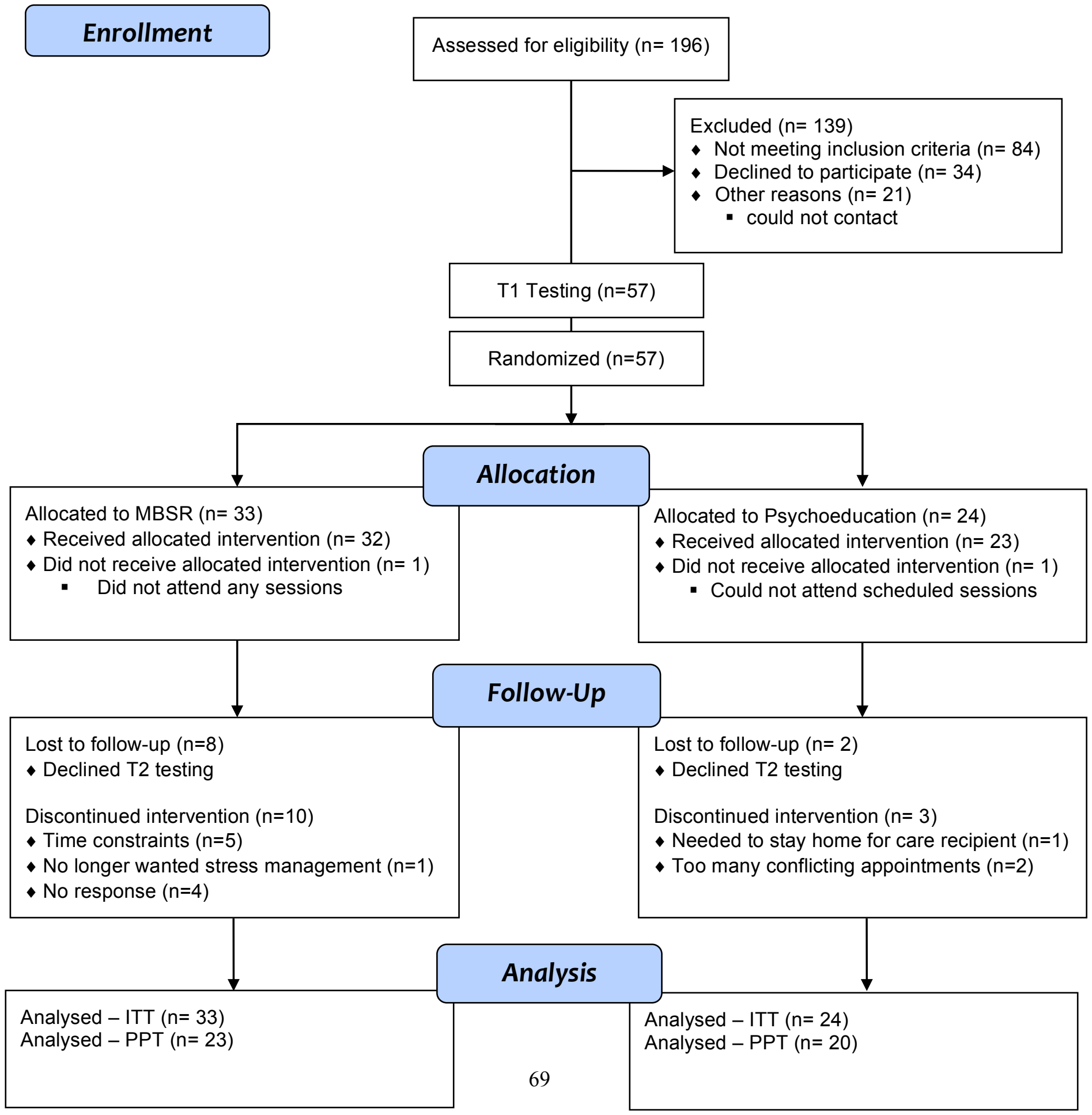


Table 4. Baseline characteristics of caregivers.

\begin{tabular}{|c|c|c|c|}
\hline Variable & $\begin{array}{l}\text { MBSR }(n=33) \\
\text { Mean }(\mathrm{SD})\end{array}$ & $\begin{array}{l}\text { Psycho-Ed }(n=24) \\
\text { Mean (SD) }\end{array}$ & $p$ \\
\hline \multicolumn{4}{|l|}{ Demographic Variables } \\
\hline Age, years & $64.18(6.69)$ & $69.13(7.86)$ & $.01 *$ \\
\hline Sex, N (\% female) & $28(85 \%)$ & $18(75 \%)$ & .35 \\
\hline Ethnicity (\% white) & $24(73 \%)$ & $20(83 \%)$ & .35 \\
\hline Education, years & $16.36(3.27)$ & $16.42(2.48)$ & .95 \\
\hline Retirement Status (\% retired) & $21(64 \%)$ & $20(83 \%)$ & .10 \\
\hline Lives with Recipient (\% yes) & $21(64 \%)$ & $17(71 \%)$ & .46 \\
\hline Years caregiving & $5.42(3.98)$ & $5.30(5.31)$ & .93 \\
\hline Care Recipient, N (\%) & & & .69 \\
\hline Parent & $11(33 \%)$ & $8(33 \%)$ & \\
\hline Spouse & $20(61 \%)$ & $14(58 \%)$ & \\
\hline Other & $2(6 \%)$ & $2(8 \%)$ & \\
\hline Disease Type, N (\%) & & & .90 \\
\hline Alzheimer's & $6(18 \%)$ & $5(21 \%)$ & \\
\hline Vascular Dementia & $4(12 \%)$ & $5(21 \%)$ & \\
\hline Parkinson's & $7(21 \%)$ & $6(25 \%)$ & \\
\hline Unknown & $12(36 \%)$ & $6(25 \%)$ & \\
\hline Other & $4(12 \%)$ & $2(8 \%)$ & \\
\hline \multicolumn{4}{|l|}{ Cognitive Variables } \\
\hline MMSE & $28.56(1.27)$ & $28.75(1.70)$ & .64 \\
\hline CVLT, Total Words & $50.06(9.46)$ & $44.29(11.27)$ & $.04 *$ \\
\hline CVLT, LDFR & $11.00(2.83)$ & $10.26(2.75)$ & .34 \\
\hline CVLT, Repetitions & $4.28(4.54)$ & $6.65(8.61)$ & .18 \\
\hline DS Forward & $6.55(1.25)$ & $6.21(1.29)$ & .33 \\
\hline DS Backward & $4.91(1.16)$ & $4.67(1.34)$ & .47 \\
\hline DS Sequencing & $5.52(1.09)$ & $5.25(1.07)$ & .37 \\
\hline TMT-A, time (s) & $36.67(13.86)$ & $43.13(29.42)$ & .27 \\
\hline TMT-B, time (s) & $85.00(32.75)$ & $90.91(37.34)$ & .53 \\
\hline TMT-B, errors & $.55(.711)$ & $1.00(1.23)$ & .12 \\
\hline TMT B-A/A & $2.40(.66)$ & $2.38(.78)$ & .91 \\
\hline Coding & $62.21(14.94)$ & $58.35(12.43)$ & .31 \\
\hline Symbol Search & $27.03(7.50)$ & $26.83(7.39)$ & .92 \\
\hline COWAT - FAS & $42.15(12.29)$ & $41.42(13.04)$ & .83 \\
\hline COWAT - Animals & $18.36(5.34)$ & $17.58(4.88)$ & .58 \\
\hline Stroop Interference $\rrbracket$ & $1.69(8.49)$ & $4.40(7.32)$ & .31 \\
\hline \multicolumn{4}{|l|}{ Psychosocial Variables } \\
\hline CESD & $19.16(10.12)$ & $19.13(12.34)$ & .99 \\
\hline RSES & $22.41(5.31)$ & $23.13(5.19)$ & .62 \\
\hline Burden & $46.97(14.07)$ & $44.48(14.31)$ & .52 \\
\hline
\end{tabular}




\begin{tabular}{|c|c|c|c|}
\hline Observe & $25.72(5.70)$ & $25.83(5.58)$ & .95 \\
\hline Describe & $27.47(5.24)$ & $28.83(5.83)$ & .37 \\
\hline Awareness & $25.81(7.36)$ & $25.57(6.37)$ & .90 \\
\hline Nonjudgment & $26.81(6.59)$ & $25.22(6.11)$ & .37 \\
\hline Nonreactive & $20.72(4.41)$ & $21.17(4.47)$ & .79 \\
\hline PSS & $23.25(5.71)$ & $21.35(7.76)$ & .30 \\
\hline QOLS & $71.66(13.31)$ & $73.65(14.72)$ & .60 \\
\hline \multicolumn{4}{|l|}{$* p<.05$} \\
\hline \multirow{2}{*}{\multicolumn{4}{|c|}{$\begin{array}{l}\text { "Note: The Stroop test was added later than other measures; } 14 \text { MBSR participants and } 23 \\
\text { Psycho-Ed participants completed this measure. }\end{array}$}} \\
\hline & & & \\
\hline \multicolumn{4}{|c|}{ MMSE $=$ Mini-Mental State Examination, CVLT $=$ California Verbal Learning Test-II, LDFR $=$} \\
\hline \multirow{2}{*}{\multicolumn{4}{|c|}{$\begin{array}{l}\text { CVLT Long Delay Free Recall, DS }=\text { Digit Span, TMT }=\text { Trail Making Test, COWAT }= \\
\text { Controlled Oral Word Association Task, CESD }=\text { Center for Epidemiologic Studies Depression }\end{array}$}} \\
\hline & & & \\
\hline \multicolumn{4}{|c|}{$\begin{array}{l}\text { Scale, RSES = Rosenberg Self-Esteem Scale, FFMQ = Five Facet Mindfulness Questionnaire, } \\
\text { PSS = Perceived Stress Scale OOLS = Ouality of Life Scale }\end{array}$} \\
\hline
\end{tabular}

\subsubsection{Cognition}

A mixed 2x2 ANCOVA using age, education, and sex as covariates, showed no significant group $\mathrm{x}$ time interactions on cognitive performance in either ITT or PPT analyses (see Table 5).

Table 5. Comparison of within- and between-group changes over time - Cognitive Function

\begin{tabular}{|c|c|c|c|c|c|}
\hline \multicolumn{2}{|c|}{ MBSR } & \multicolumn{2}{|c|}{ Control } & \multirow{2}{*}{$\begin{array}{l}\text { Interaction } \\
\text { term } p\end{array}$} & \multirow{2}{*}{$\begin{array}{l}\text { Partial } \\
\eta^{2}\end{array}$} \\
\hline $\begin{array}{l}\text { Post- } \\
\text { intervention, } \\
\text { Mean (SD) }\end{array}$ & $\begin{array}{l}\text { Change from } \\
\text { baseline, } \\
\text { Mean (SE) }\end{array}$ & $\begin{array}{l}\text { Post- } \\
\text { intervention, } \\
\text { Mean (SD) }\end{array}$ & $\begin{array}{l}\text { Change } \\
\text { from } \\
\text { baseline, } \\
\text { Mean (SE) }\end{array}$ & & \\
\hline
\end{tabular}

ITT Analyses

$\begin{array}{lllllll}\text { CVLT, LDFR } & 11.76(3.64) & .80(.55) & 10.65(2.80) & -.05(.63) & .35 & .022 \\ \text { CVLT, Repetitions } & 4.64(3.79) & -.16(1.17) & 5.55(4.69) & -1.85(1.11) & .09 & .070 \\ \text { DS Forward } & 6.76(1.27) & .20(.25) & 5.95(1.24) & -.429(.24) & .10 & .065 \\ \text { DS Backward } & 5.00(1.35) & .16(.26) & 4.52(.87) & -.33(.25) & .35 & .022 \\ \text { DS Sequencing } & 5.83(.96) & .46(.22) & 5.33(.91) & .14(.24) & .34 & .023 \\ \text { TMT-A, time (s) } & 32.88(11.71) & -1.68(2.35) & 33.76(10.72) & -4.10(2.86) & .85 & .001 \\ \text { TMT-B, time (s) } & 77.00(27.89) & -2.52(4.32) & 86.50(27.81) & -.60(4.56) & .62 & .006 \\ \text { TMT-B, errors } & .76(1.33) & .28(.34) & .75(.97) & -.15(.26) & .56 & .009 \\ \text { TMT B-A/A } & 2.43(.75) & .04(.22) & 2.63(.59) & .16(.15) & .77 & .002 \\ \text { Coding } & 65.16(13.60) & 1.04(1.30) & 63.35(10.52) & 3.20(1.12)^{*} & .15 & .052 \\ \text { Symbol Search } & 28.12(7.03) & .44(.827) & 30.50(6.71) & 2.40(1.14) & .06 & .086 \\ \text { COWAT - FAS } & 47.36(14.79) & 4.36(1.64)^{*} & 46.71(14.06) & 3.14(1.36)^{*} & .74 & .003 \\ \text { COWAT - } & 21.16(6.24) & 2.04(.72)^{*} & 18.81(5.25) & 1.10(.80) & .16 & .048 \\ \text { Animals } & & & & & & .009\end{array}$


PPT Analyses

\begin{tabular}{|c|c|c|c|c|c|c|}
\hline CVLT, LDFR & $12.09(3.19)$ & $1.09(.56)$ & $10.33(2.72)$ & $-.50(.62)$ & .15 & .059 \\
\hline CVLT, Repetitions & $4.77(3.77)$ & $.09(1.28)$ & $5.06(4.36)$ & $-2.22(1.24)$ & .07 & .093 \\
\hline DS Forward & $6.82(1.26)$ & $.18(.29)$ & $6.05(1.27)$ & $-.37(.25)$ & .15 & .057 \\
\hline DS Backward & $5.00(1.31)$ & $.09(.26)$ & $4.53(.91)$ & $-.37(.26)$ & .50 & .013 \\
\hline DS Sequencing & $5.86(.99)$ & $.50(.21)^{*}$ & $5.37(.90)$ & $.16(.24)$ & .19 & .047 \\
\hline TMT-A, time (s) & $31.86(11.24)$ & $-1.09(2.64)$ & $33.53(11.23)$ & $-1.32(2.20)$ & .72 & .004 \\
\hline TMT-B, time (s) & $77.86(29.37)$ & $-.23(4.75)$ & $86.06(28.94)$ & $1.44(3.89)$ & .61 & .007 \\
\hline TMT-B, errors & $.82(1.40)$ & $.32(.39)$ & $.72(.96)$ & $-.22(.24)$ & .56 & .010 \\
\hline TMT B-A/A & $2.51(.75)$ & $.06(.25)$ & $2.64(.59)$ & $.08(.17)$ & .95 & .000 \\
\hline Coding & $66.09(13.41)$ & $1.59(1.41)$ & $64.33(9.89)$ & $3.00(1.24)^{*}$ & .40 & .021 \\
\hline Symbol Search & $28.14(6.87)$ & $.50(.95)$ & $31.44(6.35)$ & $2.17(1.24)$ & .10 & .076 \\
\hline COWAT - FAS & $47.91(13.25)$ & $5.09(1.69)^{* *}$ & $47.74(14.41)$ & $4.00(1.35)^{*}$ & .65 & .006 \\
\hline COWAT - & $20.95(5.61)$ & $2.18(.82)^{*}$ & $18.74(5.51)$ & $.95(.89)$ & .19 & .048 \\
\hline \multicolumn{7}{|l|}{ Animals } \\
\hline Stroop Interference & $.31(7.50)$ & $-1.41(4.63)$ & $4.09(7.00)$ & $.55(1.48)$ & .71 & .006 \\
\hline
\end{tabular}

Within groups significance: $* p<.05, * * p<.01, * * * p<.001$

MMSE $=$ Mini-Mental State Examination, CVLT $=$ California Verbal Learning Test-II, LDFR $=$ CVLT Long Delay Free Recall, TMT $=$ Trail Making Test, COWAT $=$ Controlled Oral Word Association Task

\subsubsection{Psychosocial Variables}

Results indicated significant improvement on all psychosocial measures within the MBSR group (see Table 6). However, in the between groups ITT analyses, there were significant group x time interactions for depression and perceived stress only, with participants in the MBSR group showing a significantly larger reduction in depression $\left(F(1,42)=4.26, p=.045\right.$, partial $\eta^{2}=.09$, medium) and perceived stress $\left(F(1,42)=5.24, p=.03\right.$, partial $\eta^{2}=.11$, medium) than the Psychoeducation group, using age, sex, and education as covariates. In PPT analyses, there was a significant group $\mathrm{x}$ time interaction for perceived stress, with participants in the MBSR group showing a significantly larger reduction in perceived stress than participants in the Psychoeducation group $(F(1,37)=6.38, p=.016)$, with a medium effect size (partial $\left.\eta^{2}=.15\right)$. The group $\mathrm{x}$ time interaction for self-esteem was on the threshold of significance, with participants in the MBSR group showing greater improvements than participants in the Psychoeducation group $(F(1,37)=4.12, p=.05)$ with a medium effect size (partial $\left.\eta^{2}=.10\right)$. 
Table 6. Comparison of within- and between-group changes over time - Psychosocial Variables

\begin{tabular}{|c|c|c|c|c|c|c|}
\hline & \multicolumn{2}{|c|}{ MBSR } & \multicolumn{2}{|c|}{ Control } & \multirow[t]{2}{*}{$p$} & \multirow[t]{2}{*}{ Partial $\eta^{2}$} \\
\hline & $\begin{array}{l}\text { Post- } \\
\text { intervention, } \\
\text { Mean (SD) }\end{array}$ & $\begin{array}{l}\text { Change from } \\
\text { baseline, Mean } \\
\text { (SD) }\end{array}$ & $\begin{array}{l}\text { Post- } \\
\text { intervention, } \\
\text { Mean (SD) }\end{array}$ & $\begin{array}{l}\text { Change from } \\
\text { baseline, } \\
\text { Mean (SD) }\end{array}$ & & \\
\hline \multicolumn{7}{|l|}{ ITT Analyses } \\
\hline CESD & $13.92(9.43)$ & $-5.96(1.76) * *$ & 17.18 (9.99) & $-1.32(1.97)$ & $.045^{*}$ & .092 \\
\hline RSES & $24.28(5.30)$ & $1.68(.80)^{*}$ & $24.23(3.92)$ & $.55(.62)$ & .10 & .064 \\
\hline Burden & $43.32(13.66)$ & $-4.96(1.94)^{*}$ & $41.14(14.86)$ & $-2.32(1.97)$ & .16 & .047 \\
\hline \multicolumn{7}{|l|}{ FFMQ } \\
\hline Observe & $29.24(4.86)$ & $3.44(.76)^{* * *}$ & $27.55(6.84)$ & $1.59(1.03)$ & .58 & .007 \\
\hline Describe & $29.88(5.16)$ & $3.20(.78)^{* * *}$ & $30.23(6.60)$ & $.96(.97)$ & .11 & .060 \\
\hline Awareness & $26.60(6.07)$ & $1.08(.94)$ & $28.05(5.33)$ & $2.09(1.15)$ & .79 & .002 \\
\hline Nonjudgment & $30.52(6.65)$ & $3.84(.85)^{* * *}$ & $27.41(6.58)$ & $2.14(.92)^{*}$ & .31 & .025 \\
\hline Nonreactive & $23.28(4.33)$ & $2.68(.58)^{* * *}$ & $22.77(4.11)$ & $1.59(.97)$ & .57 & .008 \\
\hline PSS & $16.64(6.40)$ & $-6.00(1.03)^{* * *}$ & $17.91(7.12)$ & $-3.23(1.04)^{* *}$ & $.03 *$ & .111 \\
\hline QOLS & $78.28(14.90)$ & $7.08(1.93)^{* *}$ & $76.68(16.61)$ & $2.59(1.92)$ & .17 & .045 \\
\hline \multicolumn{7}{|l|}{ PPT Analyses } \\
\hline CESD & $13.91(9.36)$ & $-6.18(1.98)^{* *}$ & $18.30(9.79)$ & $-1.55(2.08)$ & .09 & .078 \\
\hline RSES & $24.68(5.03)$ & $2.14(.82)^{*}$ & $23.70(3.70)$ & $.50(.67)$ & .05 & .100 \\
\hline Burden & $43.14(13.16)$ & $-5.14(2.05)^{*}$ & 43.15 (13.73) & $-1.95(2.01)$ & .21 & .042 \\
\hline \multicolumn{7}{|l|}{ FFMQ } \\
\hline Observe & $29.45(4.19)$ & $3.50(.86)^{* *}$ & $27.60(6.70)$ & $2.00(1.11)$ & .78 & .002 \\
\hline Describe & $29.95(4.55)$ & $3.32(.89)^{* *}$ & $29.60(6.50)$ & $1.15(1.05)$ & .15 & .055 \\
\hline Awareness & $26.41(6.00)$ & $1.96(.96)$ & $28.00(5.41)$ & $2.50(1.16)^{*}$ & .98 & .000 \\
\hline Nonjudgment & $30.59(6.77)$ & $4.50(.88)^{* * *}$ & $26.35(5.87)$ & $2.20(1.00)^{*}$ & .17 & .050 \\
\hline Nonreactive & $23.32(3.66)$ & $2.68(.66)^{* *}$ & $22.80(4.29)$ & $2.10(.94)^{*}$ & .86 & .001 \\
\hline PSS & $16.55(6.36)$ & $-6.86(1.02) * * *$ & $18.40(7.24)$ & $-3.25(1.03) * *$ & $.02 *$ & .147 \\
\hline QOLS & $77.50(14.96)$ & $6.86(2.16)^{* *}$ & $74.55(15.85)$ & $2.65(2.05)$ & .25 & .036 \\
\hline
\end{tabular}




\section{CHAPTER EIGHT}

\section{Discussion}

Given the rapidly aging population in Canada and the developed world (United Nations, 2015), the number of family members and spouses who are taking on caregiving responsibilities for a loved one with a neurodegenerative disease is drastically increasing (Sinha, 2013). Previous studies have indicated that caregiving can have numerous negative effects on older adult caregivers' health and well-being, including cognitive and psychological consequences (Ory et al., 1999; Vitaliano et al., 2009). However, very few studies have evaluated how MBSR, a popular psychosocial intervention, may support cognitive and mental health in this population.

The first study in this dissertation compared caregivers with a community sample of older adults on various cognitive and psychological measures, in order to replicate the consistent finding that caregiving is associated with poorer cognitive and psychosocial outcomes. The second study consisted of a randomized controlled trial, comparing an MBSR protocol with a psychoeducation control intervention, in order to explore its relative efficacy in improving cognitive function and psychosocial well-being in caregivers. Although several previous studies have described the potential benefits of mindfulness training in caregivers (Hou et al., 2014; Whitebird et al., 2013) and older adults (Lenze et al., 2014) separately, this is the first study to use the Kabat-Zinn (1990) MBSR guidelines and include an active control intervention to examine cognitive and psychosocial outcomes among older adult caregivers. With regard to Study 1, although these results do not definitively suggest that caregiving 'causes' impairment, the findings reinforce the notion that caregiving is detrimental to cognitive and psychosocial well-being. Results of the RCT provide preliminary evidence for the selective benefits of MBSR relative to psychoeducation in this group of older caregivers. 


\subsection{Effects of Caregiving on Psychosocial Well-being}

The first goal was to replicate previous evidence that caregivers report significantly poorer well-being relative to non-caregivers (e.g., Esterling et al., 1994; Pinquart \& Sörenson, 2003). Consistent with the hypothesis, results demonstrated that caregivers reported greater perceived stress and depressive symptoms and lower quality of life compared with a community sample of older adults. These findings are comparable with previous research. Pinquart and Sörenson (2003) conducted a meta-analysis on the psychological and health consequences of caregiving. They integrated 84 studies and demonstrated that caregivers reported feeling significantly more stressed and depressed, and reported lower levels of subjective well-being, physical health, and self-efficacy than controls. Canadian research demonstrates that $28 \%$ of caregivers in Canada report caregiving to be "somewhat" or "very" stressful, and 35\% report feeling "overwhelmed" (Sinha, 2013). Ory et al. (1999) also showed that in a sample of over 1,500 caregivers, caregivers of persons with a diagnosed dementia or symptoms of confusion and forgetfulness (similar to the present sample), reported significant perceived strain.

The finding that $60 \%$ of caregivers in the current study were identified as at risk for clinical depression, having met the clinical cut-off score on the CES-D (i.e., 16 or greater) is important. This percentage is significantly higher than the national average reported by the Canadian Study of Health and Aging (1994) over 20 years ago, which suggested that depression is present in $16 \%$ of informal caregivers assisting someone with mild dementia at home, $40 \%$ of informal caregivers caring for someone with moderate dementia at home, and $18.4 \%$ of informal caregivers assisting someone with severe dementia in an institution. The present sample's mean score on the CES-D (19 out of 60) is also higher than caregiver samples in other more recent studies. Schulz et al. (2003) found that in a sample of 217 informal caregivers of persons with 
dementia in the United States, the average CES-D score was 15.8. Their sample characteristics were similar to the current sample, characterized as $84 \%$ female, aged 65 years $(\mathrm{SD}=13.3)$, with approximately $50 \%$ of their sample caring for a spouse. In an otherwise similar sample of 134 caregivers of persons with dementia in Hong Kong, the average CES-D score was 12.93 (SD = 9.25; Au et al., 2009). Considering the relatively high prevalence of depression in the current sample, it is possible the current sample of caregivers may have been caring for a more complex set of care recipients than in other studies. However, as care recipient's functioning was not evaluated, this explanation is speculative and deserves further inquiry.

Cameron, Cheung, Streiner, Coyte, and Stewart (2006) examined the effects of caregiving on depressive symptoms in a sample of caregivers of stroke survivors, many of whom had subsequent cognitive impairment. In their predominantly female sample of 94 caregivers with a mean age of 60.8 years $(S D=15.41), 44.7 \%$ of participants reported clinically significant depressive symptoms on the CES-D. The authors reported that elevated depressive symptoms were associated with being female, greater lifestyle interference resulting from caregiving responsibilities, decreased mastery, providing less assistance to the care recipient, and greater behavioural and psychological symptoms in the care recipient. This study illustrates the effect of depression not only on the caregivers themselves, but also on the care recipient. The cost of depression in Canada is approximately $\$ 32.3$ billion per year (Stonebridge \& Sutherland, 2016). With as many as $60 \%$ of caregivers endorsing clinically significant depressive symptoms, implementing evidence-based interventions such as MBSR in this population would not only support caregivers' well-being, but also serve to reduce their impact on the economy.

Overall subjective quality of life has been less studied in this population. In healthy populations, the average score on the QOLS is 90 (Burckhardt \& Anderson, 2003). Thus, it 
follows that the community control group had a mean QOLS score of $86.89(S D=12.50)$. However, the caregivers had a mean score of $72.49(S D=13.82)$, which is significantly lower than the controls. This finding is also clinically meaningful because previous findings have suggested that the caregiver's quality of life is significantly related to the care recipient's quality of life (Thomas et al., 2006). Thus, exploring methods to improve caregiver quality of life is imperative.

\subsection{Effects of Caregiving on Cognitive Function}

As reviewed previously, the literature indicates that relative to non-caregivers, caregivers report increased chronic stress, depression, and lower subjective well-being (Pinquart \& Sörenson, 2003). Given that each of these factors is independently associated with impaired cognitive function (Lupien et al., 2007; Perrine et al., 1995; Vitaliano et al., 2009), it was hypothesized that caregivers would have significantly lower scores on measures of executive function relative to controls. Contrary to expectations, there were no statistically significant group differences on the main outcome measure (time to complete TMT-B) or measures of episodic memory. However, among the supplementary measures of executive function, several significant between-groups differences were found: caregivers made significantly more errors on the TMT-B, named fewer words on both components of the COWAT (phonemic and semantic fluency), and learned fewer words over repeated trials on the CVLT-II.

The observation that caregivers showed statistically significant impairments relative to controls in several measures of executive function but no differences on a measure of episodic memory, suggests that executive functions may be more vulnerable to the effects of psychological distress than episodic memory. Indeed, empirical evidence suggests that while architectural changes to the hippocampus typically require several weeks of stress exposure to 
occur, neurons in the prefrontal cortex can begin to transform in as little as a single exposure (McEwen, 2008). Of note, the terms 'frontal' and 'executive' are not interchangeable. However, there is consensus in the empirical literature that executive functions (e.g., attention, working memory, task-switching, verbal fluency, and decision-making) are strongly associated with frontal structures (Alvarez \& Emory, 2006). As mentioned, the frontal lobes also have a high density of cortisol receptors, which makes this region particularly vulnerable to the detrimental effects of chronic stress (McEwen, 1998). Indeed, exposure to chronic stress has been shown to correlate negatively with grey matter volume in the PFC (Gianaros et al., 2007; Radley \& Morrison, 2005) and impair executive functions such as working memory (Lewis, Nikolova, Chang, \& Weekes, 2008), selective attention (Skosnik, Chatterton, Swisher, \& Park, 2000), and decision-making (Porcelli \& Delgado, 2009). As such, it is possible that the current sample is displaying early observable effects of chronic stress on frontal lobe function.

Although the mean completion time on the TMT-B among caregivers was not statistically significantly slower than the community sample, the mean was trending in the expected direction. Notably, however, caregivers made significantly more errors on the test. With regard to the discrepancy between TMT-B completion time and TMT-B errors, several studies have suggested that TMT-B errors are actually a more sensitive measure of executive dysfunction than completion time. In an effort to explore how TMT error rates and completion time scores might assist with diagnostic decision-making between cognitively normal controls, mild cognitive impairment, and AD, Ashendorf et al. (2008) recruited 526 older adults and grouped them as per the three diagnostic categories. All participants completed a neuropsychological battery, including the TMT. The authors found that completion time and error scores were frequently in disagreement. For instance, $71.9 \%$ of participants in the AD group committed at least 1 TMT-B 
error, whereas only $52.6 \%$ had impaired completion time scores. The authors suggested that errors were more sensitive to pathological performance than time to completion.

In order to explore which specific cognitive operations underlie TMT errors, Mahurin et al. (2006) explored the correlates of TMT-B error scores versus completion time, and found that slower completion times were more likely explained by impairments in visuomotor scanning, while errors were uniquely associated with tests of mental tracking and working memory factors associated with executive function. In a similar analysis, Amieva et al. (1998) found that in a sample of participants with AD the majority (67\%) of errors on the TMT-B were directly related to deficits in inhibition, a main aspect of executive function. Specifically, errors resulted from difficulty suppressing the automatic tendency to draw a line to the item closest to their pencil, as well as difficulty inhibiting the desire to connect letters with letters and numbers with numbers instead of switching between the two. Stuss et al. (2001) examined TMT performance in patients with lesions in either frontal-specific or non-frontal regions of the brain. Although patients with frontal lesions showed slower TMT-B completion times relative to non-frontal patients, error analysis was a more sensitive measure for distinguishing between patients in the two lesion groups. Specifically, all participants who made at least one error on TMT-B had a frontal lesion (Stuss et al., 2001).

Participants in the present study also showed relative impairment in both phonemic and semantic fluency. Tasks of verbal fluency are thought to place considerable demands on executive processes because they require participants to efficiently organize verbal information, self-monitor (i.e., keep track of responses), engage in effortful retrieval of information, and inhibit responses (Rosser \& Hodges, 1994). The phonemic fluency component of the COWAT is typically thought to be related to the left frontal region of the brain (Baldo, Schwartz, Wilkins, \& 
Dronkers, 2006; Baldo, Shimamura, Delis, Kramer, \& Kaplan, 2001). This area is hypothesized to mediate the executive processes of suppressing habitual responses to verbal categories (Perret, 1974) and active search and retrieval of information (Baldo \& Shimamura, 1998), providing further evidence that caregivers showed impaired executive function relative to the controls.

Caregivers also showed relative impairment on the semantic component of the task, which is considered more demanding on the medial temporal area of the brain - the same brain areas related to semantic memory and retrieval (Baldo et al., 2006; Pihlajamaki et al., 2000). This finding is at odds with caregivers' performance on the CVLT-II delayed recall task, which is also hypothesized to tap into this brain region. One possible reason for this discrepancy is that semantic fluency is thought to draw on executive processes more than long delay free recall. Indeed, there is great debate in the literature about whether impairment in semantic fluency is a result of degradation of the semantic store or disruption of executive function (Henry \& Crawford, 2004).

To address this question, Henry and Crawford (2004) conducted a meta-analysis of 31 studies examining verbal fluency performance in adults with focal lesions in the frontal, temporal, or non-frontal regions; and healthy adults with no neurological or psychiatric diagnoses. A total of 1,791 participants were included. Results indicated that semantic fluency is indeed sensitive to focal temporal lesions, as patients with temporal pathology were considerably (although not statistically significantly) more impaired on semantic fluency than patients with frontal lesions. However, Henry and Crawford (2004) also note that both semantic and phonemic fluency were equally sensitive to frontal dysfunction, suggesting that semantic fluency, like phonemic fluency, does draw on executive processes. Specifically, Henry and Crawford (2004) suggest that despite its unique dependence on intact semantic memory, semantic fluency 
presumably requires the same executive process that mediate phonemic fluency (e.g., initiation, efficient organization, and self-monitoring). Thus, it appears that in the current study, the relative impairment caregivers showed on semantic fluency may be mediated by its reliance on executive function, given that caregivers showed no impairment on delayed recall component of the CVLT-II.

The final measure that significantly differed between caregivers and controls was the total amount of words learned over the five CVLT-II learning trials. Relative to the delayedrecall component of the CVLT-II, which is a measure of verbal retention, the learning component has generally been considered to be more sensitive to executive function (Tremont, Halpert, Javorsky, \& Stern, 2000). This is because the CVLT-II list is unstructured and requires participants to actively organize the information in order to efficiently encode it.

To validate this assumption, Tremont et al. (2000) examined neuropsychological test results from 96 patients identified from archival files of a university medical centre. When patients were classified into groups based on the number of impaired executive tests (zero, one, two, or three), analyses revealed that patients with zero impaired executive tests performed significantly better on the learning trials than the other three groups. Moreover, of all subscales on the CVLT, Total Words Learned was most highly correlated with other measures of executive function (i.e., COWAT, TMT-B, and Wisconsin Card Sorting Test-Perseverative Responses). The authors propose that these findings provide evidence for the role of executive function in active learning and encoding, particularly when the stimuli are disorganized (Tremont et al., 2000).

Overall, these results provide compelling evidence that caregiving is associated with impairments on measures of caregivers' ability to perform complex, executive functions. These 
findings are clinically significant for a number of reasons. Tierney et al. (2001) demonstrated that TMT-B was the most important neuropsychological predictor of ADL dysfunction in a sample of older adults with and without dementia. They also reported that impairment in executive functions (e.g., cognitive flexibility) affects a person's ability to function safely. For instance, taking medications as prescribed involves more than just remembering to take them - it also involves taking them at the right time, in the right sequence, in the right circumstances, and having working knowledge about the purpose of each medication (Tierney et al., 2001).

For older adults who are also caregivers, the implications are significantly greater, since caregivers are additionally responsible for the care recipient. Although no studies to my knowledge have examined the relationship between executive function and the quality of caregiving behaviours specifically, studies have shown that in healthy older adults as well as older adults with mild cognitive impairment, executive dysfunction is negatively associated with performance in iADLs (e.g., preparing meals, handling the finances, driving, and other daily activities; Marshall et al., 2011), and caregivers of persons with dementia report spending more time helping their care recipient with iADLs than caregivers of persons with other medical disorders (Pinquart \& Sörensen, 2003). Studies have also shown that caregivers' executive function is related to their ability to accurately report the care recipient's ADL performance - an important responsibility for which physicians heavily rely on caregivers (Dassel \& Schmitt, 2008).

In a study of the ecological validity of executive function measures, Burgess, Alderman, Evans, Emslie, and Wilson (1998) conducted a neuropsychological evaluation of 92 neurological patients and 216 healthy controls, using a variety of measures of executive function including the TMT and the COWAT. Each participant's collateral (i.e., a family member or caregiver) also 
provided subjective feedback about the participant's day-to-day dysexecutive symptoms using the Dysexecutive Questionnaire (DEX). The collateral's responses on the DEX were compared with the participants' neuropsychological test results. The authors found that TMT and COWAT scores were significantly associated with the collateral's overall rating on the DEX, as well as on the DEX Inhibition subscale (which relates to the person's ability to suppress a habitual response). The COWAT also loaded on to the Executive Memory subscale, which corresponds with the inability to recall the correct order of events, monitoring, and perseveration (Burgess et al., 1998).

With regard to the ecological validity of the CVLT-II learning trials, Kibby, SchmitterEdgecombe and Long (1998) found that in a sample of adults with closed head injury, this subscale of the CVLT was significantly correlated with employment status (i.e., full-time, parttime, or unemployed), quality of performance on the job, and occupational status (i.e., unskilled labourer, professional, etc.). For caregivers, difficulty with inhibition, executive memory, and learning could lead to serious issues such as juggling appointments and meetings, difficulty recounting events to physicians and inhibiting irrelevant information, thinking quickly and creatively to solve novel problems, etc.

Overall, these findings point to the importance of researching preventative interventions that focus on caregivers. Despite being significantly younger than the control group, caregivers showed significantly poorer performance on several measures of executive function and psychological well-being. Historically, much of the health care system's attention has been placed on the patient with a neurodegenerative disease and not his or her caregiver. These data contribute to a growing body of literature that consistently points to the need for immediate action to improve the welfare of caregivers. Mindfulness-based interventions may be part of a 
solution to this severe problem.

\subsection{Effects of MBSR on Caregivers' Psychosocial Well-being}

The hypothesis that MBSR would produce significantly greater pre-to-post intervention improvements than the control group in trait mindfulness, perceived stress, depressive symptoms, self-esteem, quality of life, and burden, was partially supported. Although participants in the MBSR group showed significant improvement in all measures, when changes were compared with the control group, only depressive symptoms and perceived stress remained significant, with medium effect sizes. In per-protocol analyses, self-esteem showed a strong trend toward significance, with a medium effect size. Thus, in contrast to the lack of group differences for cognitive performance, MBSR showed robust and specific benefits for improving mood and perceived stress, and to a lesser extent, self-esteem.

With regard to the effects of MBSR on depressive symptoms, the present finding is consistent with results from meta-analyses that report on the impact of MBSR on mood in clinical and non-clinical samples (Grossman et al., 2004). In terms of the clinical significance of the effects of MBSR on depressive symptoms, the current results demonstrate that at baseline, participants in both intervention groups were above the threshold of being at risk for clinical depression (i.e., 16 or greater on the CESD) on average. However, upon completion of the intervention, MBSR participants fell below the threshold but control participants did not. Overall, the medium effect size found in the present study is consistent with the medium effect size found in a meta-analysis of MBSR and health outcomes (Grossman et al., 2004). Coupled with the statistical and clinical significance of the current finding, the results support the value of MBSR for depressive symptom reduction in caregivers. 
These findings also provide compelling support for the benefits of MBSR on perceived stress. The caregivers in this sample reported equivalent levels of perceived stress at baseline. Perceived stress was one of only two psychosocial variables that also significantly improved from baseline to follow-up in the control group (the other variable was nonjudgment on the FFMQ), which adds further support for the superiority of MBSR for stress reduction. Given the lack of between-groups differences in trait mindfulness, it is unclear whether increases in mindfulness were responsible for the improvements in perceived stress and depressive symptoms. Of note, in a sample of healthy older adults who had undergone an eight week MBSR program, Fiocco and Mallya (2015) used multivariate regression analyses, adjusting for age, education, and sex, to demonstrate that higher trait mindfulness was significantly associated with a more positive (i.e., lower) stress profile.

These findings have important clinical implications. Previous research has linked midand late-life depression and chronic stress with an increased risk of developing dementia (Markesbery et al., 2005; Saczynski et al., 2010). In a review of the literature, Byers and Yaffe (2011) propose that depression may be a risk factor for, or early symptom of, dementia. Although there are various mechanisms that may underlie the relationship between depression and dementia, Byers and Yaffe (2011) suggest that one of the primary links is increased cortisol production, whereby depressive symptoms, like chronic perceived stress, lead to increased cortisol production. As described previously, increased levels of cortisol can result in impairment to the negative feedback loop that regulates cortisol production, leading to chronic elevation of cortisol levels (Sapolsky et al., 1986). Prolonged exposure to high levels of cortisol is neurotoxic and can result in significant structural and functional changes to the brain (Conrad, 2008). Evidence of this relationship can be seen in older adults with $\mathrm{AD}$, who have high levels of 
circulating cortisol (Jenike \& Albert, 1984) and reduced hippocampal volume (Du et al., 2001). The current sample showed decreased cognitive performance relative to controls, as well as clinically significant levels of depressive symptoms and perceived stress at baseline. Thus, the finding that MBSR can significantly reduce depressive symptoms and perceived stress is especially important because of the implications for potentially reducing the risk of caregivers developing cognitive impairment in the future.

Although it did not reach strict statistical significance, it is worth mentioning that among intervention completers, MBSR participants reported greater improvement in self-esteem than control participants $\left(p=.05\right.$, partial $\eta^{2}=.10$, medium). This finding is noteworthy because previous data have suggested that self-esteem moderates the relationship between age and cognitive function, in that increased age and lower cognitive performance were associated only in a low self-esteem group (Pruessner, Lord, Meaney, \& Lupien, 2004). Moreover, self-esteem has been found to be an important correlate of hippocampal volume and stress reactivity. Specifically, in a sample of older adults, Pruessner et al. (2005) found that low levels of selfesteem and internal locus of control were significantly correlated with reduced hippocampal volume. Again, self-esteem moderated age-related patterns of cognitive function, cortisol regulation, and total brain volume, whereby low self-esteem increased the risk for poorer outcomes on these variables (Pruessner et al., 2005). Thus, although MBSR was not associated with significant improvement on hippocampal-dependent cognitive tasks in the current study, improvements in self-esteem may protect against hippocampal functional decline in the future.

In contrast to the positive effects of MBSR on perceived stress and depressive symptoms, MBSR did not appear to improve quality of life and burden relative to control. One possible explanation for this finding is that perceived stress and depressive symptoms are reflections of 
the participants' subjective responses to their situation, whereas quality of life and burden are more related to the person's objective circumstance. The QOLS asks participants to describe six domains of quality of life: physical and material well-being, relationships with other people, social and civic activities, personal development, and independence (Flanagan, 1978). The Burden Interview asks participants to rate how caregiving responsibilities may affect their health, personal, social, and financial well-being (Zarit et al., 1980). Thus, participants' objective quality of life and burden levels may not have changed significantly as a function of group assignment, but it may be postulated that participants in the MBSR group learned to better manage their general perceptions of stress and depressive feelings.

It is important to emphasize that the lack of between-groups differences in quality of life and burden should not reflect a shortcoming of MBSR, as participants reported statistically significant improvement in these measures upon completion of the group. Rather, the lack of between groups differences may be a testament to the power of the control group, as participants in the control group also reported improvement on each of the above measures (albeit not statistically significant improvement). This result suggests that to some degree, caregivers benefited from the psychoeducational materials and social interaction offered in the group. When considering the literature, this outcome is not completely unexpected (e.g., Sörensen et al., 2002).

Participants in the control group were provided with evidence-based and practical strategies to improve quality of life and reduce burden. It is highly likely that gaining this new information (e.g., understanding how dementia affects the brain, learning about and connecting with community resources, recognizing and reducing burnout, promoting healthy work-life balance, etc.) contributed to a small improvement in well-being. As per a meta-analysis by 
Sörensen et al. (2002), psychoeducation interventions do typically result in improvements in caregiver burden, depression, and ability and knowledge about caregiving (Sörensen et al., 2002). There was also significant variability in the psychosocial data, which may partly account for the unfounded between-groups differences. Nonetheless, although MBSR resulted in clear improvements in these psychosocial variables, they did not surpass the effects of the control group.

\subsection{Effects of MBSR on Caregivers' Cognitive Function}

The final hypothesis of this study was that MBSR would produce significantly greater pre-to-post intervention improvements in cognitive flexibility than the psychoeducation group (as measured by TMT-B). This hypothesis was unsupported as there were no statistically significant group-by-time interactions on any cognitive measure in either ITT or PPT analyses. This finding was unexpected, given the empirical evidence that mindfulness-based interventions can improve executive functions in older adults (e.g., Lenze et al., 2014; Moynihan et al., 2013; Newberg et al., 2010). However, only two studies have explored the effects of meditation on executive function relative to an active control condition in a sample of older adult informal caregivers of persons with dementia (i.e., Lavretsky et al., 2013; Oken et al., 2010), and the results are mixed.

The study conducted by Lavretsky et al. (2013) differed from the present study in several respects. Specifically, Lavretsky et al. (2013) examined the effects of KK versus a relaxation control group on mental health, cognitive function, and telomerase activity among informal older adult caregivers. Both groups lasted for eight weeks. The most obvious difference between the current study and Lavretsky et al.'s (2012) investigation is the mindfulness intervention: the KK condition involved 12 minutes of daily finger gesturing, mantra chanting, and breathing 
relaxation accompanied by visualization of light. The short duration of the practice may have positively impacted the results, giving caregivers the confidence and practical ability to incorporate the practice into their busy schedules. In contrast, the present study required participants to leave their care recipients to attend the group once per week for 2 hours at a time (plus travel time), and practice the techniques at home for 30 minutes each day. The requirements necessary to learn mindfulness meditation using the MBSR structured format may have proven to be too difficult for the caregivers with respect to time commitment, which may have deterred any beneficial cognitive outcomes. Although the current protocol decreased the duration of each session ( 2 hours versus 2.5 hours) and the home practice ( 30 minutes vs. 45 minutes) for this reason, it is possible that further revisions are necessary for this population. The moderating effect of practice time on cognitive outcomes will be explored in future analyses.

In addition to the difference in intervention protocol, a number of methodological differences should be highlighted that may explain Lavresky et al.'s (2012) positive findings. Prior to enrolling in Lavretsky et al.'s (2012) study, all interested participants were exposed to samples of the meditation and relaxation practices, to confirm their amenability to potentially being enrolled in either intervention. This exposure to treatment may have established a buy-in for interested participants. Although participants in the current study also had to be agreeable to participating in either intervention, some participants expressed disappointment in the group to which they were assigned. As such, it is possible that Lavretsky et al.'s sample may have been more enthusiastic about the interventions at baseline than the current sample (who had no prior exposure to the interventions). As a further potential limitation to their recruitment strategies, Lavretsky et al. (2013) did not report whether interested participants were excluded if they 
currently or previously practiced meditation. Thus, their sample may have had more meditation experience than the current sample.

In their statistical analyses, Lavretsky et al. (2013) report using ANCOVAs (controlling for age) and using one-sided $p$ values to compare post-intervention difference scores in cognitive variables, which may increase the risk of Type I (i.e., false positive) error. Additionally, their analysis did not use education or sex as covariates, which are known to impact cognitive performance (Brayne \& Calloway 1990; Deary et al., 2009; Tombaugh, 2004). Results of their ANCOVA for TMT-B demonstrate that although the KK group did show an impressive improvement on TMT-B completion time after completing the intervention (on average participants completed the test 11.2 seconds faster), the control group unexpectedly completed the task 9.9 seconds slower relative to baseline (Lavretsky et al., 2013). The authors do not report whether the within-groups changes were statistically significant. Thus, it is possible that the significant interaction was partly driven by the unexpected impairment in the control group, particularly in the context of a small sample size and because participants in both groups performed statistically equivalently on TMT-A.

The present study is more methodologically similar to the study by Oken et al. (2010), who examined the effects of 7 weeks of MBCT, psychoeducation, and respite on cognitive function in a sample of older adult caregivers. Similar to the present study, participants in the two active interventions were required to attend weekly group sessions and engage in home practice, although the number of weekly sessions and the duration of each session were somewhat shorter than the current study (i.e., seven vs. eight weeks; and 90 vs. 120 minutes, respectively). Average duration of assigned home practice was not reported. Oken et al. (2010) measured episodic memory, attention (alerting and executive control), and inhibition. Unlike the 
present study, Oken et al. only analyzed and reported results from the intervention completers (i.e., 28 out of 31 participants) and did not complete an ITT analysis. Applying the ITT analysis principle provides an unbiased assessment of the efficacy of the intervention and informs community-based decisions about the effectiveness of the treatment protocol (West \& Spring, 2002).

Similar to the present study, Oken et al. (2010) showed no significant group differences on immediate or delayed recall, or executive control. MBCT and psychoeducation outperformed the respite intervention on alerting scores, but whether MBCT outperformed psychoeducation was not reported. The three groups differed significantly on Stroop interference, but no post hoc tests were reported so presumably, the MBCT and Psychoeducation groups showed statistically equivalent improvement on this measure (Oken et al., 2010). As previously discussed, having a larger sample and including sex and education as covariates may have impacted their findings. Nonetheless, this finding more closely mirrors the current results in terms of mindfulness and psychoeducation having similar effects on cognition.

Although not a primary hypothesis, we did find that on its own, MBSR was associated with significant improvement on certain measures of executive function (i.e., verbal fluency and working memory); however, these changes were not significant when compared with changes seen in the control group. This finding converges with that of Oken et al. (2010), and suggests that although MBSR may provide limited support for executive function, it may not be the most efficacious method. As Knight, Bean, Wilton, and Lin (2015) suggest, "Until mindfulness becomes a trait, rather than a state, the benefits may not be sustained" (p. 1384). That is, it may take ongoing practice to cultivate a level of mindfulness that translates to cognitive 
improvement. Accordingly, follow-up data is needed to determine whether cognitive benefits emerge following continued practice, beyond the eight week period.

In the present study, the psychoeducation support group was designed in part to control for social support. Caregivers often report missing social contact and social support, and report high levels of social isolation (Brodaty \& Hadzi-Pavlovic, 1990). Caregivers also report sacrificing their recreational activities and hobbies, limiting social activities, and reducing or withdrawing from employment to accommodate their caregiving responsibilities (Brodaty \& Hadzi-Pavlovic, 1990). Thus, participation in either one of the eight week interventions offered in this study may have significantly contributed to the caregivers' sense of social support, which is also known to improve cognitive function (Seeman, Lusignolo, Albert, \& Berkman, 2001).

For example, in a longitudinal examination of the impact of social relationships and social support on cognitive function in older adults, Seeman et al. (2001) found that more social support was not only related to better baseline cognitive performance, but it also predicted better cognitive function 7.5 years later. In a similar study design, Crooks, Lubben, Petitti, Little, and Chiu (2008) prospectively explored whether social networks could protect against the incidence of dementia in older women. After adjusting for several confounding variables, women with larger social networks were $74 \%$ less likely to develop dementia at 4 years follow-up than women with smaller networks. Although it is unclear how quickly cognitive function can change in response to social engagement, it is possible that in the current study, exposure to social support in the psychoeducation group reduced the magnitude of the hypothesized group differences in cognitive function. Future investigations may wish to ask participants to report on the importance of social engagement within group interventions to confirm or rule out the importance of this factor for group-based interventions. 
Participants in both groups also learned about the importance of physical health and a wholesome diet - through yoga, mindful walking, and mindful eating in the MBSR group, and via modules on Exercise and Nutrition in the psychoeducation group. Indeed, some of the most commonly recommended strategies to support cognitive functioning in older adults include adhering to a Mediterranean diet (Féart et al., 2009) and engaging in moderate aerobic exercise (Colcombe \& Kramer, 2003). The importance of a nutritious diet was explicitly discussed in both interventions. The Mediterranean and similar diets are thought to be beneficial to cognitive function because they combine several nutrients that preserve neuronal structure and function, such as monounsaturated fatty acids through healthy oils and fish (Solfrizzi et al., 2006), vitamins $B_{12}$ and folate through grains and legumes (Reynolds, 2006), and antioxidants through fruit and vegetable intake (e.g., vitamin E, carotenoids, flavonoids; Engelhart, Geerlings, \& Ruitenberg, 2002). In terms of engagement in exercise, a meta-analytic study showed that introducing moderate levels of cardiovascular exercise into the lives of older adults is unequivocally associated with improvements in working memory, mental switching, and inhibiting irrelevant information - components of executive function (Colcombe \& Kramer, 2003).

Although neither of the interventions in this study required participants to actively adhere to a specific diet or exercise regimen, previous data suggest that simply learning about principles of healthy behaviours is enough to enact behavioural change. Kelley and Abraham (2004) conducted an RCT to evaluate the efficacy of a theory-based intervention on promoting healthy eating and physical activity behaviours in older adults visiting an outpatient clinic. Participants were randomized to receiving either a healthy living booklet containing persuasive arguments for engaging in healthy diet and exercise behaviours, or a patient satisfaction questionnaire. Health 
behaviours were measured on a questionnaire at baseline and at 2-weeks follow-up. Participants who read the booklet reported significantly more success in actively engaging in more healthful diet and exercise behaviours than controls (Kelley \& Abraham, 2004). Thus, in the current study, learning about the importance of proper nutrition and exercise may have led participants in both groups to make minor but significant changes to their lifestyle, partly contributing to the equivalent gains in cognitive function. Given that this hypothesis is based on speculation, future studies could include a measure of health behaviours to account for this potential mediator.

\subsection{Study Strengths}

The current study has several strengths. First, this is the first RCT to compare an eight week MBSR protocol to an active control group in a sample of older adult informal caregivers. The psychoeducation control group was chosen primarily because psychoeducation interventions are the most widely used interventions for caregivers, and represent a "treatment as usual" form of intervention (Acton \& Kang, 2001). Second, the group controlled for the effects of social interaction with other caregivers, the effects of contact with a researcher, duration of weekly sessions, time away from the care recipient, and cognitive stimulation. Third, the study methodology was superior to other studies of cognitive function in caregivers, as described in the previous section. Lastly, this study included the most comprehensive battery of psychological and cognitive measures in the literature.

\subsection{Limitations and Future Directions}

Although this study presents several compelling findings, some limitations need to be considered when interpreting the results. Importantly, there was no respite-only control group. As Oken et al. (2010) suggest, respite is commonly advocated by health care practitioners and it may be more easily accessed than an MBSR program. Similarly to the present study, Oken et al. 
found that their psychoeducation and mindfulness groups were equally effective in several respects. Including the respite arm provided them with evidence that mindfulness was at least better than a pragmatic control intervention (Oken et al., 2010). With respect to the present study, a respite-only control arm would have helped to elucidate whether the intervention benefits arose from taking a 2-hour break from caregiving. It would have also helped to determine whether attending a specific intervention is more beneficial than participating in any other activity a caregiver might choose to do during a 2-hour respite session (e.g., grocery shopping, sleeping).

Additionally, no follow-up data were reported. Follow-up measurement is important for determining whether the immediate effects of the intervention persist into the participant's life well after the group has finished. Long-term follow-up also explores the potential barriers to ongoing practice. To address these limitations, all participants are currently being invited to participate in a 1-year post-intervention session to measure whether the psychosocial benefits have been maintained.

The reported analyses also prevent us from identifying mediating and moderating factors (e.g., living with care recipient, duration of caregiving, mood, mindfulness, etc.) that may help to explain the findings. Due to sample size constraints, it was not possible to adjust for all exploratory covariates (e.g., the caregiver's relationship to the recipient, the care recipient's diagnosis, the caregiver's retirement status, etc.). The covariates used in the final analyses (age, education, sex) were theory-driven and essential to include even in spite of the loss of degrees of freedom.

This study was powered on the results of Lavretsky et al.'s (2013) study, as it used the same primary outcome variable as this study (TMT-B completion time). However, as described, 
Lavretsky et al.'s study employed poor methodology and their main significant finding on TMTB appeared to be based on impaired performance in the control group rather than substantial improvement in the meditation group. Given the dearth of literature in this area, it was important for the current study to strike a balance between conducting a power analysis for statistical rigour, and the potential limitations of basing the analysis on a study with poor methodology.

Given the large number of ANOVAs conducted in this study, the risk of obtaining a Type I error (i.e., a false positive) increased. In order to reduce the risk of the results being misinterpreted, effect sizes and $p$-values were reported for each test. All significant findings had a medium effect size, which suggests that the findings were not likely due to chance. Moreover, the findings were based on theory-driven and a priori hypotheses.

In terms of the sample, the majority of the sample ethnically identified as white/Caucasian and lived in southern Ontario, which limits the generalizability of the results to other ethnicities and cultures. Additionally, the caregivers who enrolled in this intervention study were motivated treatment-seekers, which limits the generalizability of the findings. One report suggested that $34 \%$ of informal caregivers of persons with dementia do not use any community services, and $25.7 \%$ use only one service; very few use multiple services (Brodaty, Thomson, Thompson, \& Fine, 2005). The main reasons for not using services were perceived lack of need, followed by resistance to receiving help, not inquiring about services, and lack of knowledge about service availability. Notably, caregivers who reported not needing services also reported high levels of burden and resentment, suggesting that perceived lack of need is not necessarily a function of adaptive coping (Brodaty et al., 2005). Thus, the present findings cannot be generalized to the many caregivers who do not perceive needing support, although as Brodaty et al. (2005) suggest, they may be the very individuals who have the greatest need for services. 


\subsection{Implications and Recommendations for Further Study}

These outcomes have significant health implications. They add to the consistent evidence that caregivers are in dire need of interventions to support their psychological and cognitive health. These results also suggest that MBSR may be an acceptable intervention to achieve this goal. When an older adult is diagnosed with dementia or other neurodegenerative disease, it is expected that the health care team will focus their efforts on the individual with the diagnosis. Given the results presented herein, it is imperative that health care practitioners also take the time to speak with the individual(s) who may go on to assume the caregiver role. Specifically, upon diagnosis, the caregiver should also be provided with a treatment plan - namely, referrals to community resources and interventions such as MBSR. An early discussion with the new caregiver may give them the opportunity to learn coping strategies before their burden grows too great (for an example of what this discussion may look like, see Parks \& Novielli, 2000). Indeed, many caregivers do not seek respite until their own health deteriorates (Sorrell \& Cangelosi, 2009).

From an economic standpoint, preventing stress-related illness is a far more practical and cost-effective strategy than treating it. Hou et al. (2014) found that caregivers who participated in an eight week MBSR program in China used Traditional Chinese Medicine services 53\% less than the self-help control group. Prospective data from 1,730 patients participating in a hospitalbased MBSR program in Ontario indicated that participation in MBSR was associated with consistent decreases in health care utilization over 1 year (Knight et al., 2015). Specific outcome variables included overall number of claims made to the Ontario Health Insurance Program (OHIP), costs to OHIP, specialist and family physician visits, and utilization of lab facilities. Of note, costs to OHIP were reduced by an average of $\$ 250$ per participant (Knight et al., 2015). 
Assuming that even a fraction of these savings could be generalized to the 8.1 million caregivers living in Canada (Sinha, 2013), the investment would be astronomical. Moreover, data suggest that when the caregiver does well, the care recipient does well (Sörensen et al., 2002), which maximizes the economic benefits even further.

Given that MBSR and caregiver research is still in its infancy, several questions remain to be resolved and more research in this area is necessary before robust conclusions can be drawn. Specifically, the current results raise questions about which aspects of MBSR are useful for caregivers. Qualitative reports by participants (not presented here) indicate that they did not have the time or resources to leave their care recipient and attend the group sessions for 2 hours per week, or complete 30 minutes of daily home practice. Accordingly, determining which aspects of the program were most beneficial to participants would allow facilitators to streamline the intervention. As discussed, Lavretsky et al.'s (2013) short 12-minute daily meditation intervention may have been more feasible for this group to implement in their daily lives. Despite its shorter length, the KK practice may be a better tool to help caregivers progress from state mindfulness to trait mindfulness because it is easier to incorporate into their day, rather than trying (and potentially failing) to regularly engage in $30+$ minute practices. Future studies may wish to conduct 'head-to-head' RCTs to explore the relative efficacy of various mindfulnessbased interventions in this group.

It would also be important for researchers to include measures of the care recipient's symptoms. Sörensen et al. (2002) noted in their meta-analysis of interventions for caregivers that psychotherapy was the only intervention that positively impacted the care recipient's symptoms, potentially because caregivers learned how to respond more effectively to their own emotions and were better equipped to respond to the care recipient's behaviours and emotions. Since 
MBSR teaches participants to practice being aware of difficult experiences with acceptance, willingness, openness, and curiosity, while refraining from evaluating, judging, changing, or terminating them (Baer et al., 2009), it is conceivable that MBSR may additionally improve the recipient's well-being.

Future studies would also benefit from including a pragmatic (i.e., respite-only) control group to assess whether the reported changes in functioning are related to regression to the mean, the effects of time, or taking a break from spending time with the care recipient. Additionally, exploring the potential mediating and moderating factors that may affect treatment efficacy is warranted. As described, the research questions in this study were not directly related to determining mediators and moderators, but future analyses of these data will explore these questions. We also recommend future researchers to conduct long-term follow-ups in order to assess whether the reported benefits of mindfulness persist well beyond completion of the group, and to determine the barriers to continued practice.

\subsection{Conclusion}

In Canada and many other countries around the world, the number of older adults is increasing. As the population ages, the number of people who are diagnosed with a neurodegenerative disease will also increase. As a result, so will the number of spouses and relatives who become informal caregivers. Previous studies have shown that relative to noncaregivers, caregivers are at greater risk of developing dementia themselves. Indeed, the current study demonstrated that relative to a community sample of older adults, caregivers showed decreased performance in learning, cognitive flexibility, and verbal fluency, and had significantly worse outcomes on measures of depressive symptoms, perceived stress, and quality of life. In light of these findings, this is the first study to evaluate the effects of a standardized 
MBSR intervention on cognitive and psychosocial factors in caregivers of patients with a neurodegenerative disease, using a randomized control design and an active control group. We showed that MBSR is a feasible intervention for this population and in particular, it may be particularly beneficial in terms of reducing perceived stress and depressive symptoms. 
Appendix A

Telephone Script for Recruiting - MISSISSAUGA-HALTON LHIN CAREGIVERS

E.g, "I would like to volunteer for your research"

"I heard about your research and would like to learn more about participating"

Older adult calls in, asking about the study.

You say:

Absolutely, let me tell you a bit about the study.

\section{You are returning a message that was left by an older adult who called in for the study. You say:}

Hello, this is [name] from Ryerson University, returning your call regarding a study we are conducting looking at the effects of a stress reduction program for caregivers. Do you have a few minutes to chat?

\section{General script:}

Caregivers are commonly considered a segment of the population who undergoes chronic stress. Due to the very nature of your role, you may be at increased risk for stress-related illness. Many caregivers report high levels of distress, poor sleep quality, feelings of depression and memory problems. All of these outcomes can significantly affect your quality of life and your role as caregiver.

We are running a study to see if a stress reduction program for caregivers of people with dementia or other neurodegenerative disorders (such as Parkinson's) can improve relationship quality, psychological well-being and thinking abilities - such as memory, attention.

So may I ask what sort of condition your loved one has that requires your care?

As a volunteer, you would be asked to meet with us on 10 occasions. The first session will last for an hour and a half. During this session, you will be asked to do paper-and-pencil tasks that assess your thinking ability - such as memory, attention, and problem solving. You will also be asked to fill out a few questionnaires that ask about things like the quality of your relationship with the person you care for, your mood and how you feel about yourself and your quality of life, and the amount of stress you experience in your daily life. We will schedule this session at a library close to your home, so that we have a quiet and private space to do the assessment.

After the first session, you will be randomly assigned to one of two stress-management programs. Because we are randomly assigning participants to one of the two groups, you cannot choose which group to participate in, nor do I have any control over which groups to assign you to.

One of the 2 programs is called Mindfulness Training (or MT). The other program is called Psychoeducational Training (PT). 
Mindfulness Training is a program that combines relaxation, light stretching and focused breathing. Everything that is done in class is tailored to your comfort level (e.g. you will not be pushed to stretch more than you can).

The Psychoeducational Training (PT) group will teach participants about resources and tools to help manage stress. Every week, caregivers will be presented with a topic of discussion including: Understanding Dementia; Caregiver Burnout; Accessing Care; Effective Communication; Life Balance; Nutrition; Fitness; and Rewards of Caregiving. Not only will participants learn new information provided by the facilitator, but they will also have a change to discuss current issues and learn from their caregiver peers.

Regardless of which group you get assigned to, there are 8 sessions in total, once a week, each lasting 2 hours. A trained facilitator will lead the group each week. Each week, you will meet with a group of no more than 10-15 people for 2 hours and you may be asked to practice the learned techniques at home every day for approximately 30 minutes a day.

We are also offering an optional session that will run for 3-hours between weeks 6 and 7. During this silent session, you will be guided through all of the skills learned during your sessions. However, it is understandable that taking this time from your normal activities may not be possible and therefore this half-day is not mandatory and only suggested.

About one week after the final stress reduction session, we will meet you once again at a local library to do a reassessment of the same kinds of thinking abilities we assessed during the first session.

Before I go on, do you have any questions about the 2 programs?

Caregiver ReCharge Service is able to assist by making a personal support worker (PSW) available to care for your loved one at home while you attend this program. This means that a PSW will come to your home for approximately 3-4 hours (2 hours for attendance and 1-2 hours for travelling time, which will vary depending on where you live with respect to workshop location). You must attend the session in order to receive the PSW. A PSW will also be provided for both testing sessions, as well. We will arrange for the PSW. Again, the amount of time will depend on the session ( 2 hours for workshop and 1.5 hours for the testing sessions) and travel time.

Are you still interested in participating?

If yes: Wonderful, before we move forward, I will have to ask you a few demographic and health-related questions.

It is important to tell you that all information you give me today is confidential. Any information that identifies who you are (e.g. your name, telephone number) will be removed from the data and will be stored in a separate locked filing cabinet. Identifying information will be destroyed once all of your data has been collected. Data kept on the computer will be password protected 
and held for 7 years after publication of study results. After that, all data will be properly destroyed. This applied for the information you give me today and for any information you give me during the study.

...[proceed to phone screening questionnaire]

If no: I understand. Thank you very much for your time and have a wonderful day.

Person does not meet criteria: I am sorry to say that you do not meet the eligibility criteria for this study. For the purpose of this study, we have excluded XX. Often in research, we have exclusion criteria, to make sure that our sample is relatively the same on certain characteristics. I am very grateful for your time and the information you provided today.

Again, all information you have given me today is confidential. The reason why we keep the information you gave me today is very important for research - it gives us a description of who we were able to recruit and who we were not able to recruit from the community. May I have your consent to use this confidential phone-screen data for this purpose? Again, your name will not be attached to the data.

Thank you for your time! 


\title{
Telephone Script for Recruiting - COMMUNITY CAREGIVERS
}

\author{
E.g, "I would like to volunteer for your research" \\ "I heard about your research and would like to learn more about participating"
}

\section{Older adult calls in, asking about the study.}

You say:

Absolutely, let me tell you a bit about the study.

\section{You are returning a message that was left by an older adult who called in for the study. You say:}

Hello, this is [name] from Ryerson University, returning your call regarding a study we are conducting looking at the effects of a stress reduction program for caregivers. Do you have a few minutes to chat?

\section{General script:}

Caregivers are commonly considered a segment of the population who undergoes chronic stress. Due to the very nature of your role, you may be at increased risk for stress-related illness. Many caregivers report high levels of distress, poor sleep quality, feelings of depression and memory problems. All of these outcomes can significantly affect your quality of life and your role as caregiver.

We are running a study to see if a stress reduction program for caregivers of people with dementia or other neurodegenerative disorders (such as Parkinson's) can improve relationship quality, psychological well-being and thinking abilities - such as memory, attention.

So may I ask what sort of condition your loved one has that requires your care?

As a volunteer, you would be asked to come to Ryerson on 10 occasions. The first session will last for an hour and a half. During this session, you will be asked to do paper-and-pencil tasks that assess your thinking ability - such as memory, attention, and problem solving. You will also be asked to fill out a few questionnaires that ask about things like the quality of your relationship with the person you care for, your mood and how you feel about yourself and your quality of life, and the amount of stress you experience in your daily life. You will be paid $\$ 25$ at the end of the session for your time.

After the first session, you will be randomly assigned to one of two stress-management programs, both of which are held at Ryerson once a week for 8 weeks in a row. Because we are randomly assigning participants to one of the two groups, you cannot choose which group to participate in, nor do I have any control over which groups to assign you to.

One of the 2 programs is called Mindfulness Training (or MT). The other program is called Psychoeducational Training (PT). 
Mindfulness Training is a program that combines relaxation, light stretching and focused breathing. Everything that is done in class is tailored to your comfort level (e.g. you will not be pushed to stretch more than you can).

The Psychoeducational Training (PT) group will teach participants about resources and tools to help manage stress. Every week, caregivers will be presented with a topic of discussion including: Understanding Dementia; Caregiver Burnout; Accessing Care; Effective Communication; Life Balance; Nutrition; Fitness; and Rewards of Caregiving. Not only will participants learn new information provided by the facilitator, but they will also have a change to discuss current issues and learn from their caregiver peers.

Regardless of which group you get assigned to, there are 8 sessions in total, once a week, each lasting 2 hours. A trained facilitator will lead the group each week. Each week, you will meet with a group of no more than 10-15 people for 2 hours and you may be asked to practice the learned techniques at home every day for approximately 30 minutes a day.

You will receive another $\$ 25$ during the $5^{\text {th }}$ session, which is considered the half-way point.

We are also offering an optional session that will run for 3-hours between weeks 6 and 7. During this silent session, you will be guided through all of the skills learned during your sessions. However, it is understandable that taking this time from your normal activities may not be possible and therefore this half-day is not mandatory and only suggested.

Before I go on, do you have any questions about the 2 programs?

...About one week after the final intervention session, you will come back to complete the same kind of tasks and questionnaires you did during the first session. You will be paid another $\$ 25$ for this session.

Are you still interested in participating?

If yes: Wonderful, before we move forward, I will have to ask you a few demographic and health-related questions.

It is important to tell you that all information you give me today is confidential. Any information that identifies who you are (e.g. your name, telephone number) will be removed from the data and will be stored in a separate locked filing cabinet. Identifying information will be destroyed once all of your data has been collected. Data kept on the computer will be password protected and held for 7 years after publication of study results. After that, all data will be properly destroyed. This applied for the information you give me today and for any information you give me during the study.

...[proceed to phone screening questionnaire]

If no: I understand. Thank you very much for your time and have a wonderful day. 


\section{Script Following phone screening questionnaire:}

Person meets criteria: Wonderful! You meet the requirements to participate in this study. Again, all information you have given me today, and all information you will provide during the study, is confidential. Any information that identifies who you are (e.g. your name, telephone number) will be removed from the data and will be stored in a separate locked filing cabinet until the end of your participation in the study at which point all identifying information will be destroyed. All de-identified information (i.e. your data) will be kept in locked cabinets or password protected on the laboratory computer and will be held for 7 years after publication of study results. After that, all data will be properly destroyed.

I will contact you shortly to book your first testing session. If you have any questions in the mean time, please do not hesitate to contact me [provide contact info]

Person does not meet criteria: I am sorry to say that you do not meet the eligibility criteria for this study. For the purpose of this study, we have excluded XX. Often in research, we have exclusion criteria, to make sure that our sample is relatively the same on certain characteristics. I am very grateful for your time and the information you provided today.

Again, all information you have given me today is confidential. The reason why we keep the information you gave me today is very important for research - it gives us a description of who we were able to recruit and who we were not able to recruit from the community. May I have your consent to use this confidential phone-screen data for this purpose? Again, your name will not be attached to the data.

Thank you for your time! 
Appendix B

\section{Phone Screening Questionnaire - CAREGIVERS}

Researcher: To determine your eligibility for the study, I will now ask you a few questions about demographic health information. Everything you say will be kept confidential. You do not have to answer any questions that you are uncomfortable with; however, you may be excluded based on the information you choose to withhold.

\section{DEMOGRAPHIC QUESTIONS}

\begin{tabular}{|c|c|}
\hline \multicolumn{2}{|l|}{ Age: } \\
\hline Sex: (Do you identify as male or female) & \\
\hline Ethnicity (e.g., Caucasian, Hispanic, etc.): & \\
\hline Country of birth: & \\
\hline Languages you speak: & \\
\hline $\begin{array}{l}\text { If English is not your mother tongue, at what } \\
\text { age did you learn to speak English? }\end{array}$ & \\
\hline Handedness: & Right__ Left__ Ambidextrous \\
\hline Are you retired? & $\begin{array}{l}\text { No } \_ \text {Yes } \\
\text {--if yes, in what year did you retire? }\end{array}$ \\
\hline How many years of education do you have? & \\
\hline $\begin{array}{l}\text { How many years have you been a primary } \\
\text { caregiver? } \\
\text { Are you a caregiver of: spouse, parent, or } \\
\text { other? } \\
\text { Do you believe that your role as a caregiver is } \\
\text { demanding? } \\
\text { Do you receive help from other family } \\
\text { members? } \\
\text { What kind of dementia does your loved one } \\
\text { have? (Alzheimer's, Frontotemporal, Lewy } \\
\text { Body, Vascular, etc.) }\end{array}$ & $\begin{array}{l}\text { Years } \\
\text { Spouse / Parent / Other } \\
\text { Yes _ No } \\
\text { Yes _ No }\end{array}$ \\
\hline $\begin{array}{l}\text { Have you ever practiced yoga, meditation, tai } \\
\text { chi, or any other similar Eastern practice? } \\
\text { Exclude is has a good understanding of } \\
\text { mindfulness meditation and have a regular } \\
\text { practice (=>3 times per week) }\end{array}$ & $\begin{array}{l}\text { Ever Practiced: Yes No } \\
\text { Current Practice: Yes No Unsure }\end{array}$ \\
\hline
\end{tabular}




\begin{tabular}{|l|l|}
\hline $\begin{array}{l}\text { Are you actively participating in any sort of } \\
\text { formal brain training activities, e.g., Lumosity, } \\
\text { Brain Metrix? }\end{array}$ & $\begin{array}{l}\text { Yos } \\
\text { Yes }\end{array}$ \\
\hline $\begin{array}{l}\text { Have you had any cognitive or } \\
\text { neuropsychological testing in the past year? }\end{array}$ & $\begin{array}{l}\text { No } \\
\text { Yes }\end{array}$ \\
\hline
\end{tabular}

\section{HEALTH-RELATED QUESTIONS} Have you ever had any of the following?

\begin{tabular}{|c|c|}
\hline $\begin{array}{l}\text { Vision problems (glasses, operations): } \\
\text { (exclude if vision is not corrected) }\end{array}$ & $\begin{array}{l}\text { No Yes } \\
\text {--If yes, specify: } \\
\text {-- If yes, is your vision corrected to } 20 / 20 \text { with } \\
\text { glasses? } \\
\text { No Yes }\end{array}$ \\
\hline $\begin{array}{l}\text { Hearing problems (weak hearing, hearing } \\
\text { aids): } \\
\text { (exclude if hearing is not corrected) }\end{array}$ & $\begin{array}{l}\text { No Yes } \\
\text {--If yes, specify: } \\
\text {-- Is your hearing corrected to normal with the } \\
\text { hearing aid? } \\
\text { No Yes }\end{array}$ \\
\hline $\begin{array}{l}\text { Psychiatric illness: } \\
\text { Have you been diagnosed with any of the } \\
\text { following? } \\
\text { Mood disorder yes / no } \\
\text { Anxiety disorder yes / no } \\
\text { Psychotic disorder yes / no } \\
\text { Dissociative disorder yes / no } \\
\text { Substance use yes / no } \\
\text { (exclude if not controlled current Axis I } \\
\text { diagnosis) }\end{array}$ & $\begin{array}{l}\text { No Yes } \\
\text {-- If yes, when were you diagnosed? } \\
\quad \text { Year: } \\
\text {-- If yes, are you currently taking medication to treat } \\
\text { your symptoms? } \\
\text { No Yes }\end{array}$ \\
\hline $\begin{array}{l}\text { Diabetes: } \\
\text { (exclude if not controlled) }\end{array}$ & $\begin{array}{l}\text { No Yes } \\
\text {-- If yes, when were you diagnosed? } \\
\text { Year: } \\
\text {-- If yes, is it controlled with medication? } \\
\text { Year: }\end{array}$ \\
\hline
\end{tabular}




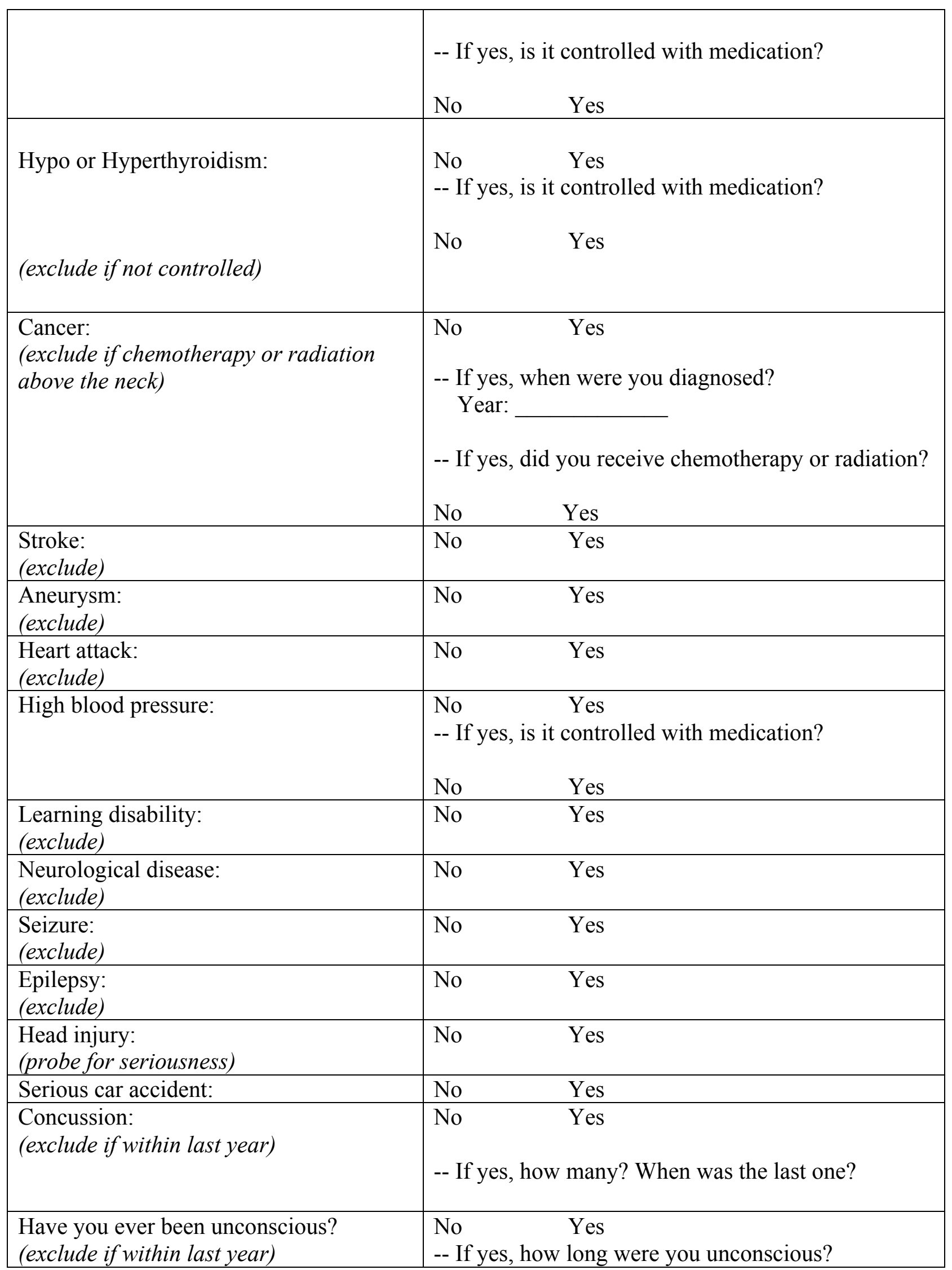




\begin{tabular}{|c|c|}
\hline & $\begin{array}{l}\text { Duration: } \\
\text {-- If yes, what was the cause? } \\
\text { Cause: }\end{array}$ \\
\hline $\begin{array}{l}\text { Have you ever had surgery that required } \\
\text { general anesthesia? } \\
\text { (exclude if within last year) }\end{array}$ & $\begin{array}{l}\text { No } \quad \text { Yes } \\
\text {-- If yes, when did you have this procedure? } \\
\quad \text { Year: }\end{array}$ \\
\hline
\end{tabular}




\title{
Ryerson University Consent Agreement COMMUNITY CAREGIVERS
}

\begin{abstract}
Study: Evaluating the effects of a stress-reduction program on relational quality, psychological well-being and cognitive function in caregivers of patients with dementia
\end{abstract}

You are being asked to participate in a research study. Before you give your consent to be a volunteer, it is important that you read the following information and ask as many questions as necessary to be sure you understand what you will be asked to do.

\section{Investigators:}

Principal Investigator: Dr. Alexandra J. Fiocco, PhD., Department of Psychology, Ryerson University

Collaborator: Sasha Mallya, MA, Department of Psychology, Ryerson University

\section{Background and Purpose of the Study:}

Approximately one-in-five Canadians who are 45 years of age or older provide care for a family member with long-term health problems. In general, caregivers are considered a group of individuals who are exposed to chronic stress due to their caregiving role; however, there are exceptions and not all caregivers experience chronic stress. A number of stress-related illnesses have been reported in caregivers, including emotional distress, depression, poor sleep quality, and overall poor quality of life. Studies show that older adult caregiving spouses show cognitive problems, such as problems with learning and memory, compared to non-caregiving spouses. Importantly, caregivers of dementia patients are reportedly six times more likely to develop dementia themselves compared to non-caregivers. While not all caregivers will experience accelerated cognitive decline, this statistic alone calls for the implementation of programs that may prevent declined in physical and mental health.

The purpose of this study is to assess whether an 8-week stress-management program enhances relationship quality, psychological well-being and cognitive function (e.g. learning and memory, attention ability) in caregivers of patients with dementia. We plan to recruit 140 caregivers aged 50-85 years from the Greater Toronto Area. To be eligible for this study, you have indicated that you are a primary informal caregiver, you do not have a diagnosed psychopathology or neurological disorder, you have not been exposed to chemotherapy or neuropsychological testing in the past year, and you do not exhibit significant vision or hearing problems. You have also indicated that you do not have experience with yoga or meditation, nor do you have a regular practice (3 or more times a week).

\section{Description of the Study:}

Participants will be asked to come to Ryerson University for 10 to 11 sessions in total: 2 testing sessions, 8 program sessions and one optional weekend session (if possible). 


\section{Two Testing Sessions:}

Cognitive testing, such as testing your memory, and questionnaires will be completed in Dr. Fiocco's Stress and Healthy Aging Research Lab at the Institute for Stress and Well Being at Ryerson University. Both sessions will take place in the morning and will last approximately 1.5 hours. Cognitive tasks will include learning and memory, tests of attention and processing speed, language fluency and abstract thinking. Self-report questionnaires will include those that assess relationship quality, psychological well-being, and quality of life.

Time commitment for testing sessions:

1.5 hours in total on each day.

\section{Eight-Week Program with optional half-day retreat:}

The Mindfulness Training (MT) program is an 8-week program consisting of weekly 2-hourlong sessions. In groups of 10-15, participants will be taught various meditation practices that will be applied in class and to routine daily activities. Participants will do relaxation exercises, walking meditation, and light stretching. All activities will be tailored to the comfort of the participant (e.g. stretching). In addition to weekly classes, participants are asked to practice their new skills at home for 30 minutes a day, six days a week. You will be given a CD to help guide you through the home practice. In addition to the 8 sessions, there is an optional "half day" session (10am-3pm) between session 6 and session 7 . The halfday retreat is not mandatory, but is highly suggested.

\section{Or}

The Psychoeducational Training (PT) group will teach participants about resources and tools to help manage stress. Every week, caregivers will be presented with a topic of discussion including: Understanding Dementia; Caregiver Burnout; Accessing Care; Effective Communication; Life Balance; Nutrition; Fitness; and Rewards of Caregiving. Not only will participants learn new information provided by the facilitator, but they will also have a change to discuss current issues and learn from their caregiver peers.

Weekly sessions in both groups will be audio recorded (except during the first session when you introduce yourself) to ensure that group facilitators are following the program protocol. A few of these recordings will be sampled and reviewed by another researcher to make sure that the facilitator is following procedure. If you disclose information during the session that you do not want to be recorded, please tell the facilitator and she will erase the recorded segment.

Time commitment for the program:

Session: 2 hours once a week, over 8 weeks

Homework: 120- 180 minutes a week, over 8 weeks (the amount of time you put into your homework is up to you; naturally the more time the better).

"Half-day": 5 hours 


\section{What is Experimental in this Study:}

The experimental component of this study is undergoing an 8-week program. By agreeing to participate, you are agreeing to be randomized to either program condition (MT or PT). This is to say that you are willing to participate in either intervention.

\section{Risks or Discomforts:}

Your participation in the first and second testing sessions may create temporary frustration or fatigue. Some of the cognitive tasks (e.g. memorizing a list of words) may be challenging, causing frustration; however, we emphasize that you are not expected to get everything correct and that we only ask you to try your best. Should you feel uncomfortable during the testing session and while filling out psychological questionnaires, you may take a break before moving on. You may also choose not to answer certain questions if you feel uncomfortable. You may also choose to stop participating altogether.

Psychological or physical discomfort may be experienced during the intervention sessions. Physical discomfort may be experienced while stretching muscles. If physical discomfort is experienced, back up and talk to the facilitator who will ensure that you execute each position with comfort. The MT and PT programs are tailored to your ability. Psychological discomfort may arise from group topics or conversations. Sometimes the mere practice of meditation may cause an emotional release. If this does occur, feel free to take a break, walk outside the room, or have some tea and cookies. The facilitator is there to help you through these emotions.

Should you decide that you are no longer interested in volunteering, you may withdraw from the study and stop participating at any time, without penalty.

\section{Benefits of the Study:}

By participating in this study, you will learn either MT or psychoeducational techniques free of charge. You will also receive material to help you with your home practice. Although direct benefits are not guaranteed, you may benefit from weekly social contact with other caregivers and from the new skills that you will learn. Overall, your participation will help assess whether stress reduction interventions can enhance physical and mental health in caregivers of patients with dementia.

\section{Confidentiality:}

All data collected for this study will remain confidential. The data that we collect from you will be stripped of all identifying information. That is, a unique study code will be assigned to your file in order to prevent any assistant from making a link between your name and your test results. Although your name will appear on this consent form, this form will be stored in a separate locked cabinet and will not be linked to your study code. All data will be securely locked in a cabinet and password protected on the laboratory computer - only Dr. Fiocco and specific trainees working on the project will have access to this data. All data will be securely stored up to 7 years following publication of results, after which all material will be properly destroyed.

While confidentiality of data collected is guaranteed, we are unable to guarantee confidentiality of discussions that take place during the intervention session. Because the researchers cannot guarantee confidentiality, we are asking all participants to be courteous and keep the sessions private. Sessions will be audio recorded, with exception of first half hour of the first session when participants may disclose their first and last name. These audio recordings are to ensure 
that the facilitators follow the program protocol. Should you disclose any information during the session that you do not want recorded, please tell the facilitator and she will erase that segment. The purpose of the recording is to assess the facilitator, not participant conversation.

\section{Incentives to Participate:}

The main incentive for participants is to experience a free stress-reduction program for 8 weeks. In addition, three payments of $\$ 25$ (\$75 total) will be administered: $\$ 25$ for each testing session and $\$ 25$ for attending the 8-week program. If you decide to stop participating after attending the first program session, you will still be entitled to compensation associated with the 8-week program.

\section{Costs and/or Compensation for Participation:}

Other than transportation, there are no costs associated with taking part in this study. All material associated with the intervention will be free of charge. As mentioned above, participants will be compensated up to $\$ 75$.

\section{Voluntary Nature of Participation:}

Participation in this study is voluntary. Your choice of whether or not to participate will not influence your future relations with Ryerson University. If you decide to participate, you are free to withdraw your consent and to stop your participation at any time without penalty or loss of benefits to which you are entitled.

At any particular point in the study, you may refuse to answer any particular question or stop participation altogether. If you decide to stop participating, all information that you provided before stopping will remain confidential in a secured database at Ryerson University and used for future analyses. This data will be kept for up to 7 years following publication of results, after which it will be destroyed.

\section{What will be done if "abnormal" findings/results are obtained:}

The tests that you will complete are not "diagnostic" - i.e. no diagnosis can be made with the tests that we administer to you. However, if there is anything about your performance on the tasks and questionnaires that raise a concern for us, we will discuss it with you.

\section{Questions about the Study:}

If you have any questions about the research now, please ask. If you have questions later about the research, you may contact the principal investigator: Alexandra J. Fiocco via phone (416979-5000 ext 3008) or email (afiocco@psych.ryerson.ca).

If you have questions regarding your rights as a human subject and participant in this study, you may contact the Ryerson University Research Ethics Board for information via phone (416-9795042) or email (rebchair@ryerson.ca). You may also write them at:

Research Ethics Board c/o Office of the Vice President, Research and Innovation

Ryerson University

350 Victoria Street

Toronto, ON M5B 2K3 


\section{Agreement:}

Your signature below indicates that you have read the information in this agreement and have had a chance to ask any questions you have about the study. Your signature also indicates that you agree to be in the study and have been told that you can change your mind and withdraw your consent to participate at any time. Your signature also indicates that you are aware that the program sessions will be recorded for assessment of the facilitator. You have been offered a copy of this agreement.

You have been told that by signing this consent agreement you are not giving up any of your legal rights.

Name of Participant (please print)

Signature of Participant

Date

Signature of Investigator/Study Coordinator

Date

Dissemination of Results:

If you agree to participate in the study, are you interested in learning about the results of the study in a final report? If so, please provide your mailing or email address below:

Email

Mailing 
RYERSON

UNIVERSITY

\title{
Ryerson University Consent Agreement
}

Study: Evaluating the effects of a stress-reduction program on relational quality, psychological well-being and cognitive function in caregivers of persons with dementia

\author{
Version: Mississauga-Halton Local Health Integration Network
}

You are being asked to participate in a research study. Before you give your consent to be a volunteer, it is important that you read the following information and ask as many questions as necessary to be sure you understand what you will be asked to do.

\section{Investigators:}

Principal Investigator: Dr. Alexandra J. Fiocco, PhD., Department of Psychology, Ryerson University

Collaborator: Sasha Mallya, MA, Department of Psychology, Ryerson University

\section{Background and Purpose of the Study:}

Approximately one-in-five Canadians who are 45 years of age or older provide care for a family member with long-term health problems. In general, caregivers are considered a group of individuals who are exposed to chronic stress due to their caregiving role; however, there are exceptions and not all caregivers experience chronic stress. A number of stress-related illnesses have been reported in caregivers, including emotional distress, depression, poor sleep quality, and overall poor quality of life. Studies show that older adult caregiving spouses show cognitive problems, such as problems with learning and memory, compared to non-caregiving spouses. Importantly, caregivers of persons with dementia are reportedly six times more likely to develop dementia themselves compared to non-caregivers. While not all caregivers will experience accelerated cognitive decline, this statistic alone calls for the implementation of programs that may prevent declines in physical and mental health.

The purpose of this study is to assess whether an 8-week stress-management program enhances relationship quality, psychological well-being and cognitive function (e.g. learning and memory, attention ability) in caregivers of persons with dementia. We plan to recruit 140 caregivers aged 50-85 years from the Greater Toronto Area. To be eligible for this study, you have indicated that you are a primary informal caregiver, you have not been exposed to chemotherapy or neuropsychological testing in the past year, and you do not exhibit significant vision or hearing problems. You have also indicated that you do not have extensive experience with yoga or meditation, nor do you have a regular practice (3 or more times a week). In order for us to judge the effectiveness of the programs, we must ensure that all participants are novices in mindfulness and progressive muscle relaxation.

\section{Description of the Study:}

Participants will be asked to meet with us for 10 to 11 sessions in total: 2 testing sessions, 8 program sessions and one weekend session (if possible). 


\section{Two Testing Sessions:}

Cognitive testing, such as testing your memory, and questionnaires will be completed at a public library close to your home. Both sessions will last approximately 1.5 hours. Cognitive tasks will include learning and memory, tests of attention and processing speed, language fluency and abstract thinking. Self-report questionnaires will include those that assess relationship quality, psychological well-being, and quality of life.

Time commitment for testing sessions:

1.5 hours in total on each day.

\section{Eight-Week Program:}

You will be randomly assigned to one of two stress-management programs. Both programs consist of weekly 2 -hour long sessions in groups of 10-15 caregivers.

1. The Mindfulness Training (MT) group will consist of learning various meditation practices that will be applied in class and to routine daily activities. Participants will do relaxation exercises, walking meditation, and light stretching. All activities will be tailored to the comfort of the participant (e.g. stretching). In addition to weekly classes, participants are asked to practice their new skills at home for 30 minutes a day. You will be given a CD to help guide you through the home practice. In addition to the 8 sessions, there is a 3-hour session between weeks 6 and 7 .

\section{Or}

2. The Psychoeducation Training group will consist of an educational component and a social support component. Every week, caregivers will be presented with a topic of discussion and will learn about resources and tools to help manage stress. The weekly topics include: Understanding Dementia; Caregiver Burnout; Accessing Care; Effective Communication; Life Balance; Nutrition; Fitness; and Rewards of Caregiving. Not only will participants learn new information provided by the facilitator, but they will also have a change to discuss current issues and learn from their caregiver peers.

Weekly sessions in both groups will be audio recorded (except during the first session when you introduce yourself) to ensure that group facilitators are following the program protocol. A few of these recordings will be sampled and reviewed by another researcher to make sure that the facilitator is following procedure. If you disclose information during the session that you do not want to be recorded, please tell the facilitator and she will erase the recorded segment.

\section{What is Experimental in this Study:}

The experimental component of this study is undergoing an 8-week program. By agreeing to participate, you are agreeing to be randomized to either program condition. This is to say that you are willing to participate in either intervention. 


\section{Risks or Discomforts:}

Your participation in the first and second testing sessions may create temporary frustration or fatigue. Some of the cognitive tasks (e.g. memorizing a list of words) may be challenging, causing frustration; however, we emphasize that you are not expected to get everything correct and that we only ask you to try your best. Should you feel uncomfortable during the testing session and while filling out psychological questionnaires, you may take a break before moving on. You may also choose not to answer certain questions if you feel uncomfortable. You may also choose to stop participating altogether.

Psychological or physical discomfort may be experienced during the intervention sessions. Physical discomfort may be experienced while tensing or stretching muscles. If physical discomfort is experienced, back up and talk to the facilitator who will ensure that you execute each position with comfort. The programs are tailored to your ability. Psychological discomfort may arise from group topics or conversations. Sometimes the mere practice of meditation may cause an emotional release. If this does occur, feel free to take a break or walk outside the room. The facilitator is there to help you through these emotions.

Should you decide that you are no longer interested in volunteering, you may withdraw from the study and stop participating at any time, without penalty.

\section{Benefits of the Study:}

By participating in this study, you will learn either MT or basic stress-management techniques free of charge. You will also receive material (readings, CD) to help you with your home practice. Although direct benefits are not guaranteed, you may benefit from weekly social contact with other caregivers and from the new skills that you will learn. Overall, your participation will help assess whether stress reduction interventions can enhance physical and mental health in caregivers of patients with dementia.

\section{Confidentiality:}

All data collected for this study will remain confidential. The data that we collect from you will be stripped of all identifying information. That is, a unique study code will be assigned to your file in order to prevent any assistant from making a link between your name and your test results. Although your name will appear on this consent form, this form will be stored in a separate locked cabinet and will not be linked to your study code. All data will be securely locked in a cabinet and password protected on the laboratory computer - only Dr. Fiocco and specific trainees working on the project will have access to this data. All data will be securely stored up to 7 years following publication of results, after which all material will be properly destroyed.

While confidentiality of data collected is guaranteed, we are unable to guarantee confidentiality of discussions that take place during the intervention session. Because the researchers cannot guarantee confidentiality, we are asking all participants to be courteous and keep the sessions private. Sessions will be audio recorded on a password-protected device, with exception of first half hour of the first session when participants may disclose their first and last name. These audio recordings are to ensure that the facilitators follow the program protocol. Should you disclose any information during the session that you do not want recorded, please tell the facilitator and she will erase that segment. The purpose of the recording is to assess the facilitator, not participant conversation. 


\section{Incentives to Participate:}

The main incentive for participants is to experience a free stress-reduction program for 8 weeks. In addition, Caregiver ReCharge Service is able to assist by making a personal support worker (PSW) available to care for your loved one at home while you attend this program. This means that a PSW will come to your home for approximately 3-4 hours (2 hours for attendance and 1-2 hours for travelling time, which will vary depending on where you live with respect to workshop location). A PSW will also be provided for both testing sessions, as well. We will arrange for the PSW. Again, the amount of time will depend on the session (2 hours for workshop and 1.5 hours for the testing sessions) and travel time.

\section{Costs and/or Compensation for Participation:}

Other than transportation, there are no costs associated with taking part in this study. All material associated with the intervention will be free of charge (e.g. CD and readings). As mentioned above, participants will be provided with a PSW during the workshop and testing sessions, if needed.

\section{Voluntary Nature of Participation:}

Participation in this study is voluntary. Your choice of whether or not to participate will not influence your future relations with Ryerson University. If you decide to participate, you are free to withdraw your consent and to stop your participation at any time without penalty or loss of benefits to which you are entitled.

At any particular point in the study, you may refuse to answer any particular question or stop participation altogether. If you decide to stop participating, all information that you provided before stopping will remain confidential in a secured database at Ryerson University and used for future analyses. This data will be kept for up to 7 years following publication of results, after which it will be destroyed.

What will be done if "abnormal" findings/results are obtained:

The tests that you will complete are not "diagnostic" - i.e. no diagnosis can be made with the tests that we administer to you. However, if there is anything about your performance on the tasks and questionnaires that raise a concern for us, we will discuss it with you.

\section{Questions about the Study:}

If you have any questions about the research now, please ask. If you have questions later about the research, you may contact the principal investigator: Alexandra J. Fiocco via phone (416979-5000 ext 3008) or email (afiocco@psych.ryerson.ca).

If you have questions regarding your rights as a human subject and participant in this study, you may contact the Ryerson University Research Ethics Board for information via phone (416-9795042) or email (rebchair@ryerson.ca). You may also write them at:

Research Ethics Board

c/o Office of the Vice President, Research and Innovation

Ryerson University

350 Victoria Street

Toronto, ON M5B 2K3 
Study: Evaluating the effects of a stress-reduction program on relational quality, psychological well-being and cognitive function in caregivers of persons with dementia

\section{Agreement:}

Your signature below indicates that you have read the information in this agreement and have had a chance to ask any questions you have about the study. Your signature also indicates that you agree to be in the study and have been told that you can change your mind and withdraw your consent to participate at any time. Your signature also indicates that you are aware that the program sessions will be recorded for assessment of the facilitator. You have been offered a copy of this agreement.

You have been told that by signing this consent agreement you are not giving up any of your legal rights.

Name of Participant (please print)

Signature of Participant

Date

Signature of Investigator/Study Coordinator

Date

\section{Dissemination of Results:}

If you agree to participate in the study, are you interested in learning about the results of the study in a final report? If so, please provide your mailing or email address below:

Email

Mailing 
Appendix D

\section{Debriefing Script}

I would like to thank you for participating in this study. Your valuable time and effort is greatly appreciated. (Alternative for drop out - see below A.)

The goal of this study is to assess the effect of mindfulness training on relationship quality, wellbeing and cognitive functions, such as memory and attention. In this study, we are comparing mindfulness training and psychoeducation, two techniques that have been down to significant decrease distress in young and older adults.

Remember, cognitive function can mean memory performance - such as remembering a list of words - or attention, or speed of completing a task. You performed these tasks before the 8week program and after. You also completed a questionnaire asking about the quality of your relationship with the person who you are caring for. Well-being was assessed with those questionnaires that you filled out regarding your mood, your perceived stress, how you felt about yourself (self-esteem) etc.

This data will be used to determine if mindfulness training, compared to psychoeducation can be used as an effective tool to decrease caregiver burden and improve cognitive function and relationship quality. While both MT and psychoeducation are shown to reduce stress, there is some new evidence in young adults that suggests mindfulness training can have positive effects on cognition. However, this has not been assessed in older individuals.

Again, thank you so much for the time and effort you put into this study. Your efforts will help us understand the usefulness of potential interventions that may prevent or slow down declines in cognitive function and overall health in caregivers.

I must stress that being a caregiver does not mean that you will necessarily experience problems with your cognitive function. There are a number of factors that lead to individual differences in physical and psychological health, which is not linked to the caregiver role. There are caregivers who are not chronically stressed and there are caregivers who do not show problems with their physical or mental health. 
Do you have any questions for me?

Remember - on the consent form that we gave you, you have the names and contact information of people to contact should you have any questions what so ever. You can contact the Research Ethics Board, who approved this study. You can also contact Dr. Alexandra Fiocco, should you have any additional questions.

\section{Dissemination of Results:}

Although we do not give out individual results, you were asked during the consent process whether or not you would be interested in learning about the results of the study in a final report. Are you still interested? (if they said no originally but yes now, take down email/mailing).

\section{SPECIFIC IF DROP OUT}

A. I would like to thank you for participating in this study up to now. Your valuable time and effort is greatly appreciated.

Before I let you go, I would like to debrief you on the study a little... (move on to above script)

Remember, you signed an informed consent before beginning the study. I would like to restate that your choice to no longer participate in this study will not influence your future relations with Ryerson University. Stopping your participation now will not penalize you in any way.

All information that you provided prior to stopping will remain in the secured database at Ryerson University for up to 7 years following publication of the results. 


\section{RYERSON}

UNIVERSITY

\section{DEBRIEFING FORM}

\section{Bringing relief to caregivers: Evaluating the effects of mindfulness-based stress reduction on relational quality, psychological well-being and cognitive function in caregivers of patients with dementia}

Thank you for participating in this study. Your time and effort are extremely appreciated. This study investigated the effect of a stress-reduction program on relationship quality, psychological well-being and cognitive function in caregivers of patients with dementia. Cognitive function includes memory, attention, and speed of processing (or how quickly you can do certain tasks).

You were asked to complete various cognitive tasks and to fill out questions related to your relationship quality, mood and overall well-being, before and after 8-weeks of participating in one of two programs: mindfulness training or psychoeducation.

Although both mindfulness training and psychoeducation are shown to be significantly effective in reducing levels of distress and stress-related symptoms (e.g. depression, sleep problems) in young and older adults, there is new evidence in young adults that mindfulness training may also improve cognitive function. The goal of this study is to see if mindfulness training can improve cognitive function in adult caregivers 50 years and older.

This study has received ethics clearance through the Psychology Department Research Ethics Committee at Ryerson University. If you have any questions about this study or concerns about your participation in this study, please contact Dr. Alexandra Fiocco at 416-979-5000-3008 or afiocco@psych.ryerson.ca. If you have any concerns about the ethical procedures of this study, please contact the Ryerson University Research Ethics Board at 416-979-5042 or rebchair@ryerson.ca.

For more information on mindfulness training, visit:

Kate Kitchen: http://www.meditation-for-mindfulness.com/meditation.html

Center for Mindfulness Studies: http://www.mindfulnessstudies.com/ 
Appendix E

\begin{tabular}{|c|c|c|c|c|c|c|c|c|c|}
\hline $\begin{array}{c}\text { Name } \\
\text { (Alphabetical by } \\
\text { First Name) }\end{array}$ & ID & Feb 2 & Feb 9 & Feb 16 & Feb 23 & Mar 1 & Mar 8 & Mar 15 & Mar 22 \\
\hline Name 1 & 1 & $\checkmark$ & $\sqrt{ }$ & $\sqrt{ }$ & $x$ & $x$ & $\sqrt{ }$ & $\sqrt{ }$ & $\sqrt{ }$ \\
\hline Name 2 & 2 & & & & & & & & \\
\hline Name 3 & 3 & & & & & & & & \\
\hline$\ldots$ & $\ldots$ & & & & & & & & \\
\hline & & & & & & & & & \\
\hline & & & & & & & & & \\
\hline & & & & & & & & & \\
\hline & & & & & & & & & \\
\hline & & & & & & & & & \\
\hline & & & & & & & & & \\
\hline & & & & & & & & & \\
\hline
\end{tabular}




\section{Appendix F \\ This week's practice \\ Sample Home Practice Record Form}

For the next 6 days, do 30 minutes of a practice of your choice (yoga, sitting mediation, body scan)

OR do 15 minutes each of any 2 practices including yoga, sitting medi or walking meditation on your own.

\begin{tabular}{|l|l|l|l}
\hline Date & Practice & $\begin{array}{l}\text { Length of timel } \\
\text { Number of times } \\
\text { practiced }\end{array}$ & \\
Time & & & \\
\hline Day 1 & & & \\
\hline Time & & & \\
\hline Day 2 3 & & & \\
Time & & & \\
\hline Time & & & \\
\hline Time & & & \\
\hline Day 5 & & & \\
\hline
\end{tabular}




\section{Appendix G \\ Facilitator Feedback Questionnaire}

Please let us know what you thought about the group facilitator:

Facilitator established good rapport by demonstrating:

1. Genuineness

\begin{tabular}{lllllll}
0 & 1 & 2 & 3 & 4 & 5 & 6 \\
\hline Poor & $\begin{array}{l}\text { Barely } \\
\text { Adequate }\end{array}$ & Mediocre & $\begin{array}{l}\text { Satis- } \\
\text { factory }\end{array}$ & Good & Very Good & Excellent
\end{tabular}

2. Warmth

\begin{tabular}{lllllll}
0 & 1 & 2 & 3 & 4 & 5 & 6 \\
\hline Poor & $\begin{array}{l}\text { Barely } \\
\text { Adequate }\end{array}$ & Mediocre & $\begin{array}{l}\text { Satis- } \\
\text { factory }\end{array}$ & Good & Very Good & Excellent
\end{tabular}

3. Accurate Empathy

\begin{tabular}{lllllcc}
0 & 1 & 2 & 3 & 4 & 5 & 6 \\
\hline Poor & $\begin{array}{l}\text { Barely } \\
\text { Adequate }\end{array}$ & Mediocre & $\begin{array}{l}\text { Satis- } \\
\text { factory }\end{array}$ & Good & Very Good & Excellent
\end{tabular}

4. Facilitator engaged with participants in a professional manner.

\begin{tabular}{llllllc}
0 & 1 & 2 & 3 & 4 & 5 & 6 \\
\hline Poor & $\begin{array}{l}\text { Barely } \\
\text { Adequate }\end{array}$ & Mediocre & $\begin{array}{l}\text { Satis- } \\
\text { factory }\end{array}$ & Good & Very Good & Excellent
\end{tabular}

5. Facilitator structured class time efficiently, and was able to keep the focus of the session.

\begin{tabular}{lllllll}
0 & 1 & 2 & 3 & 4 & 5 & 6 \\
\hline Poor & $\begin{array}{l}\text { Barely } \\
\text { Adequate }\end{array}$ & Mediocre & $\begin{array}{l}\text { Satis- } \\
\text { factory }\end{array}$ & Good & Very Good & Excellent
\end{tabular}


6. Facilitator elicited feedback about the participants' reactions to the course material.

\begin{tabular}{lllllcc}
0 & 1 & 2 & 3 & 4 & 5 & 6 \\
\hline Poor & $\begin{array}{l}\text { Barely } \\
\text { Adequate }\end{array}$ & Mediocre & $\begin{array}{l}\text { Satis- } \\
\text { factory }\end{array}$ & Good & Very Good & Excellent
\end{tabular}

7. Facilitator assigned homework in a clear and specific manner.

\begin{tabular}{lllllll}
0 & 1 & 2 & 3 & 4 & 5 & 6 \\
\hline Poor & $\begin{array}{l}\text { Barely } \\
\text { Adequate }\end{array}$ & Mediocre & $\begin{array}{l}\text { Satis- } \\
\text { factory }\end{array}$ & Good & Very Good & Excellent
\end{tabular}

8. Facilitator asked participants about anticipated problems with completing homework, and problem solved to resolve them.

\begin{tabular}{lllllll}
0 & 1 & 2 & 3 & 4 & 5 & 6 \\
\hline Poor & $\begin{array}{l}\text { Barely } \\
\text { Adequate }\end{array}$ & Mediocre & $\begin{array}{l}\text { Satis- } \\
\text { factory }\end{array}$ & Good & Very Good & Excellent
\end{tabular}




\section{Intervention Feedback Form}

\section{Please let us know what you thought about the 8-week program:}

1. Did you find this intervention enjoyable? Yes / No

Additional comment(s):

2. If applicable, did you find the homework helpful? Yes / No / Not Applicable Additional comment(s):

3. Did you think the duration of the program was appropriate with respect to:

a. 8-weeks? Yes / No

b. 2 hours each week? Yes / No

Additional comment(s):

4. Are there any changes that you feel would make this intervention better?

5. If any, what was/were the biggest benefit(s) you received from the program?

6. If any, what was/were the biggest challenge(s) in completing the program? 
7. Did the program impact your physical health? If so, please explain.

8. Did the program impact your emotional well-being? If so, please explain.

9. How often were you practicing your new skills at home DURING the 8-week program (e.g., once per week for 3 hours; twice per day for 20 minutes; etc.)?

10. How often have you been practicing your new skills at home since the program FINISHED (e.g., once per week for 3 hours; twice per day for 20 minutes; etc.)?

11. How likely is it that you will continue to practice your new skills in the future?

\section{Thank you for your feedback!}




\section{References}

Acton, G. J., \& Kang, J. (2001). Interventions to reduce the burden of caregiving for an adult with dementia: A meta-analysis. Research in Nursing \& Health, 24, 349-360. doi: 10.1002/nur.1036

Adelman, R. D., Tmanova, L. L., Delgado, D., Dion, S., \& Lachs, M. S. (2014). Caregiver burden: A clinical review. Journal of the American Medical Association, 311(10), 10521060. doi:10.1001/jama.2014.304

Akpinar, B., Küçükgüçlü, Ö., \& Yener, G. (2011). Effects of gender on burden among caregivers of Alzheimer's patients. Journal of Nursing Scholarship, 248-254. doi: 10.1111/j.1547-5069.2011.01402.x

Alberts, N. M., Hadjistavropoulos, H. D., Pugh, N. E., \& Jones, S. L. (2011). Dementia anxiety among older adult caregivers: an exploratory study of older adult caregivers in Canada. International Psychogeriatrics, 23(06), 880-886. doi: 10.1017/

S1041610211000299

Alvarez, J. A., \& Emory, E. (2006). Executive function and the frontal lobes: A meta-analytic review. Neuropsychology Review, 16(1), 17-42. doi: 10.1007/s11065-006-9002-x

Alzheimer Society of Canada. (2010). Rising tide: The impact of dementia on Canadian society. Ottawa, ON: Author.

Alzheimer Society of Canada. (2014). Facts about dementia. Ottawa, ON: Author.

Alzheimer Society of Canada. (2015). Frontotemporal dementia. Retrieved from http://www.alzheimer.ca/en/About-dementia/Dementias/Frontotemporal-Dementia-andPick-s-disease

Alzheimer Society of Canada. (2016). Prevalence and monetary costs of dementia. Toronto, 
ON: Author.

Alzheimer Society of Canada. (2017). National dementia strategy. Retrieved from http://www.alzheimer.ca/en/Get-involved/Advocacy/National-dementia-strategy Amieva, H., Lafont, S., Auriacombe, S., Rainville, C., Orgogozo, J. M., Dartigues, J. F., \& Fabrigoule, C. (1998). Analysis of error types in the Trail Making Test evidences an inhibitory deficit in dementia of the Alzheimer type. Journal of Clinical and Experimental Neuropsychology, 20(2), 280-285.

Anderson-Hanley, C., Arciero, P. J., Brickman, A. M., Nimon, J. P., Okuma, N., Westen, S. C., ... \& Zimmerman, E. A. (2012). Exergaming and older adult cognition: a cluster randomized clinical trial. American Journal of Preventive Medicine, 42(2), 109-119. doi: 10.1016/j.amepre.2011.10.016

Angus, D. E., Auer, L., Cloutier, J. E., \& Albert, T. (1995). Cost-effectiveness in ambulatory care: Alternative approaches. The Journal of Ambulatory Care Management, 18(1), 2938.

Ashendorf, L., Jefferson, A. L., O’Connor, M. K., Chaisson, C., Green, R. C., \& Stern, R. A. (2008). Trail Making Test errors in normal aging, mild cognitive impairment, and dementia. Archives of Clinical Neuropsychology, 23(2), 129-137. doi:

10.1016/j.acn.2007.11.005

Astin, J. A. (1997). Stress reduction through mindfulness meditation. Psychotherapy and Psychosomatics, 66, 97-106. doi: 10.1159/000289116

Au, A., Lai, M. K., Lau, K. M., Pan, P. C., Lam, L., Thompson, L., \& Gallagher-Thompson, D. (2009). Social support and well-being in dementia family caregivers: The mediating role of self-efficacy. Aging \& Mental Health,13(5), 761-768. doi: 


\section{$10.1080 / 13607860902918223$}

Azulay, J., Mott, T., Cicerone, K. D., \& Smart, C. M. (2012). An open trial of mindfulnessbased stress reduction (MBSR) with a mixed brain injury population. Brain Injury, 26(45), 588 .

Baer, R. A. (2003). Mindfulness Training as a Clinical Intervention: A Conceptual and Empirical Review. Clinical Psychology: Science and Practice, 10, 125-143. doi: 10.1093/clipsy.bpg015

Baer, R. A., Smith, G. T., Hopkins, J., Krietemeyer, J., \& Toney, L. (2006). Using self-report assessment methods to explore facets of mindfulness. Assessment, 13, 27-45. doi: $10.1177 / 1073191105283504$

Baikie, E. (2002). The impact of dementia on marital relationships. Sexual and Relationship Therapy, 17, 289-299. doi:10.1080/14681990220149095

Baldo, J. V., Schwartz, S., Wilkins, D., \& Dronkers, N. F. (2006). Role of frontal versus temporal cortex in verbal fluency as revealed by voxel-based lesion symptom mapping. Journal of the International Neuropsychological Society, 12(06), 896-900. doi: 10.10170S1355617706061078

Baldo, J. V., \& Shimamura, A. P. (1998). Letter and category fluency in patients with frontal lobe lesions. Neuropsychology, 12(2), 259-267.

Baldo, J. V., Shimamura, A. P., Delis, D. C., Kramer, J., \& Kaplan, E. (2001). Verbal and design fluency in patients with frontal lobe lesions. Journal of the International Neuropsychological Society, 7(05), 586-596.

Barry, D., Bates, M. E., \& Labouvie, E. (2008). FAS and CFL forms of verbal fluency differ in difficulty: a meta-analytic study. Applied Neuropsychology, 15(2), 97-106. doi: 


\section{$10.1080 / 09084280802083863$}

Bauer, M. E., Vedhara, K., Perks, P., Wilcock, G. K., Lightman, S. L., \& Shanks, N. (2000). Chronic stress in caregivers of dementia patients is associated with reduced lymphocyte sensitivity to glucocorticoids. Journal of Neuroimmunology, 103(1), 84-92. doi: 10.1016/S0165--5728(99)00228-8-3

Beck A. (1975). Cognitive therapy and the emotional disorders. Madison, CT: International Universities Press.

Beck A. (1993). Cognitive Therapy and the Emotional Disorders. New York: Penguin.

Benton, A. L. (1989). Multilingual aphasia examination. Iowa City, Iowa: AJA Associates Bergdahl, J., \& Bergdahl, M. (2002). Perceived stress in adults: Prevalence and association of depression, anxiety and medication in a Swedish population. Stress and Health, 18, 235241. doi: $10.1002 /$ smi.946

Biegel, D., Sales, E., \& Schulz, R. (1991). Family caregiving in chronic illness: Heart disease, cancer, stroke, Alzheimer's disease, and chronic mental illness. Newbury Park, CA: Sage Publications.

Birnie, K., Garland, S. N., \& Carlson, L. E. (2010). Psychological benefits for cancer patients and their partners participating in mindfulness-based stress reduction (MBSR). PsychoOncology, 19, 1004-1009. doi: 10.1002/pon.1651

Bishop, S. R., Lau, M., Shapiro, S., Carlson, L., Anderson, N. D., Carmody, J., ... Devins, G. (2004). Mindfulness: A Proposed Operational Definition. Clinical Psychology: Science and Practice, 11(3), 230-241. doi: 10.1093/clipsy.bph077

Borders, A., Earleywine, M., \& Jajodia, A. (2010). Could mindfulness decrease anger, hostility, and aggression by decreasing rumination? Aggressive Behavior, 36, 28-44. doi: 
$10.1002 / \mathrm{ab} .20327$

Brayne, C., \& Calloway, P. (1990). The association of education and socioeconomic status with the Mini Mental State Examination and the clinical diagnosis of dementia in elderly people. Age and Ageing, 19(2), 91-96.

Breytspraak, L. M., \& George, L. K. (1979). Measurement of self-concept and self-esteem in older people: State of the art. Experimental Aging Research, 5(2), 137-148.

Brodaty, H., \& Hadzi-Pavlovic, D. (1990). Psychosocial effects on carers of living with persons with dementia. Australian and New Zealand Journal of Psychiatry, 24(3), 351-361.

Brodaty, H., Roberts, K., \& Peters, K. (1994). Quasi-experimental evaluation of an educational model for dementia caregivers. International Journal of Geriatric Psychiatry, 9, 195204. doi: $10.1002 / g p s .930090304$

Brodaty, H., Thomson, C., Thompson, C., \& Fine, M. (2005). Why caregivers of people with dementia and memory loss don't use services. International Journal of Geriatric Psychiatry, 20(6), 537-546. doi: 10.1002/gps.1322

Bronskill, S. E., Carter, M. W., Costa, A. P., Esensoy, A. V., Gill, S. S., Gruneir, A., ... \& Wodchis, W. P. (2010). Aging in Ontario: An ICES chartbook of health service use by older adults. Toronto: Institute for Clinical Evaluative Sciences.

Brown, K. W., \& Ryan, R. M. (2003). The benefits of being present: Mindfulness and its role in psychological well-being. Journal of Personality and Social Psychology, 84, 822-848. doi: $10.1037 / 0022-3514.84 .4 .822$

Burgess, P. W., Alderman, N., Evans, J., Emslie, H., \& Wilson, B. A. (1998). The ecological validity of tests of executive function. Journal of the International Neuropsychological Society, 4(06), 547-558. 
Burckhardt, C. S., \& Anderson, K. L. (2003). The Quality of Life Scale (QOLS): Reliability, validity, and utilization. Health and Quality of Life Outcomes, 1(60), 1-7. doi: $10.1186 / 1477-7525-1-60$

Burckhardt, C. S., Woods, S. L., Schultz, A. A., \& Ziebarth, D. M. (1989). Quality of life of adults with chronic illness: A psychometric study. Research in Nursing \& Health, 12(6), 347-354.

Butcher, H., Holkup, P., \& Buckwalter, K. (2001). The experience of caring for a family member with Alzheimer's disease. Western Journal of Nursing Research, 23, 33-55. doi: $10.1177 / 019394590102300104$

Byers, A. L., \& Yaffe, K. (2011). Depression and risk of developing dementia. Nature Reviews Neurology, 7(6), 323-331. doi: 10.1038/nrneurol.2011.60

Cameron, J. I., Cheung, A. M., Streiner, D. L., Coyte, P. C., \& Stewart, D. E. (2006). Stroke survivors' behavioral and psychologic symptoms are associated with informal caregivers' experiences of depression. Archives of Physical Medicine and Rehabilitation, 87(2), 177-183. doi: 10.1016/j.apmr.2005.10.012

Canadian Institute for Health Information. (2007)., The burden of neurological diseases, disorders and injuries in Canada. Ottawa: CIHI.

Canadian Study of Health and Aging Working Group. (1994). Patterns of caring for people with dementia in Canada. Canadian Journal on Aging, 13, 470-487.

Cantor, M. H. (1991). Family and community: changing roles in an aging society. The Gerontologist, 31, 337-346. doi: 10.1093/geront/31.3.337

Capistrant, B. D., Moon, J. R., \& Glymour, M. M. (2012). Spousal caregiving and incident hypertension. American Journal of Hypertension, 25, 437-43. doi: 10.1038/ajh.2011.232 
Carlson, M. C., Fried, L. P., Xue, Q. L., Bandeen-Roche, K., Zeger, S. L., \& Brandt, J. (1999). Association between executive attention and physical functional performance in community-dwelling older women. The Journals of Gerontology Series B: Psychological Sciences and Social Sciences, 54(5), S262-S270. doi: 10.1093/geronb/54B.5.S262

Carlson, L. E., Speca, M., Patel, K. D., \& Goodey, E. (2003). Mindfulness-based stress reduction in relation to quality of life, mood, symptoms of stress, and immune parameters in breast and prostate cancer outpatients. Psychosomatic Medicine, 65, 571581. doi: 10.1097/01.PSY.0000074003.35911.41

Caswell, L., Vitaliano, P., \& Croyle, K. (2003). Negative associations of chronic stress and cognitive performance in older adult spouse caregivers. Experimental Aging, 29, 303318. doi: $10.1080 / 03610730303721$

Chambers, R., Lo, B. C. Y., \& Allen, N. B. (2008). The impact of intensive mindfulness training on attentional control, cognitive style and affect. Cognitive Therapy \& Research, 32, 303-322. doi: 10.1007/s10608-007-9119-0

Chien, W. T., Chan, S. W., \& Morrissey, J. (2007). The perceived burden among Chinese family caregivers of people with schizophrenia. Journal of Clinical Nursing, 16, 1151-1161. doi: 10.1111/j.1365-2702.2007.01501.x

Chiesa, A., \& Serretti, A. (2009). Mindfulness-based stress reduction for stress management in healthy people: A review and meta-analysis. The Journal of Alternative and Complementary Medicine, 15, 593-600. doi: 10.1089/acm.2008.0495

Chiverton, P., \& Caine, E. D. (1989). Education to assist spouses in coping with Alzheimer's Disease: A controlled trial. Journal of the American Geriatrics Society, 37, 593-598. doi: 10.1111/j.1532-5415.1989.tb01248.x 
Cohen, C. A., Pringle, D., \& LeDuc, L. (2001). Dementia caregiving: The role of the primary care physician. The Canadian Journal of Neurological Sciences, 28, S72-S76. doi: $10.1017 / \mathrm{S} 0317167100001232$

Cohen, S. (1986). Contrasting the hassles scale and the perceived stress scale: Who's really measuring appraised stress? American Psychologist, 41, 717-718.

Cohen, S., Kamarch, T., \& Mermelstein, R. (1983). A global measure of perceived stress. Journal of Health and Social Behavior, 24(4), 385-396.

Colcombe, S., \& Kramer, A. F. (2003). Fitness effects on the cognitive function of older adults: a meta-analytic study. Psychological Science, 14(2), 125-130. doi: 10.1111/14679280.t01-1-01430

Conrad, C. D. (2008). Chronic stress-induced hippocampal vulnerability: the glucocorticoid vulnerability hypothesis. Reviews in the Neurosciences, 19(6), 395-412. doi:

\subsection{5/REVNEURO.2008.19.6.395}

Cotman, C. W., \& Berchtold, N. C. (2002). Exercise: a behavioral intervention to enhance brain health and plasticity. Trends in Neurosciences, 25, 295-301. doi: 10.1016/S0166$2236(02) 02143-4$

Cranswick, K., \& Dosman, D. (2008). Eldercare: What do we know? Canadian Social Trends. (Statistics Canada Catalogue no. 11-008-x).

Crawley, M. J. (2005). Statistics: An introduction using R. West Sussex, UK: John Wiley \& Sons.

Crooks, V. C., Lubben, J., Petitti, D. B., Little, D., \& Chiu, V. (2008). Social network, cognitive function, and dementia incidence among elderly women. American Journal of Public Health, 98(7), 1221-1227. doi: 10.2105/AJPH.2007.115923 
Crowe, S. F. (1998). The differential contribution of mental tracking, cognitive flexibility, visual search, and motor speed to performance on parts A and B of the trail making test. Journal of Clinical Psychology, 54(5), 585-591. doi:10.1002/(SICI)10974679(199808)54:5<585::AID-JCLP4>3.0.CO;2-K

Dassel, K. B., \& Schmitt, F. A. (2008). The impact of caregiver executive skills on reports of patient functioning. The Gerontologist, 48(6), 781-792. doi:10.1093/geront/48.6.781

Davids, T. \& Stede, W. (Eds.) (1921/2001). Pali-english dictionary. New Delhi, India: Munshiram Manoharlal Publishers Pvt, Ltd.

Deary, I. J., Corley, J., Gow, A. J., Harris, S. E., Houlihan, L. M., Marioni, R. E., ... \& Starr, J. M. (2009). Age-associated cognitive decline. British Medical Bulletin, 92(1), 135-152. doi: $10.1093 / \mathrm{bmb} / \mathrm{ldp} 033$

Delis, D., Kaplan, E., Kramer, J., \& Ober, B. (2000). California Verbal Learning Test-II. San Antonio, TX: The Psychological Corporation

Delis, D. C., Massman, P. J., Kaplan, E., Mckee, R., Kramer, J. H., \& Gettman, D. (1991). Alternate form of the California Verbal Learning Test: Development and reliability. The Clinical Neuropsychologist, 5, 154-162. doi: 10.1080/13854049108403299

Desbiens, N. A., Mueller-Rizner, N., Virnig, B., \& Lynn, J. (2001). Stress in caregivers of hospitalized oldest-old patients. The Journals of Gerontology Series A: Biological Sciences and Medical Sciences, 56, M231-M235. doi: 10.1093/ gerona/56.4.M231

de Vugt, M. E., Jolles, J., van Osch, L., Stevens, F., Aalten, P., Lousberg, R., et al. (2006). Cognitive functioning in spousal caregivers of dementia patients: Findings from the prospective MAASBED study. Age and Ageing, 35, 160-166. doi: 10.1093/ageing/afj044 
Doherty, C. P., Hutchinson, S., Abrahams, S., \& Coen, R. F. (2011). Frontotemporal Dementia. In Neurodegenerative Disorders (pp. 115-142). Springer: London.

Dupuis, S., Epp, T., \& Smale, B. (2004). Caregivers of persons with dementia: Roles, experiences, supports and coping: A literature review. Murray Alzheimer Research and Education Program, 1-112.

Engelhart, M. J., Geerlings, M. I., Ruitenberg, A., van Swieten, J. C., Hofman, A., Witteman, J. C., \& Breteler, M. M. (2002). Dietary intake of antioxidants and risk of Alzheimer disease. Journal of the American Medical Association, 287(24), 3223-3229. doi: 10.1001/jama.287.24.3223

Esterling, B. A., Kiecolt-Glaser, J. K., Bodnar, J. C., \& Glaser, R. (1994). Chronic stress, social support, and persistent alterations in the natural killer cell response to cytokines in older adults. Health Psychology, 13, 291-298. doi: 10.1037/0278-6133.13.4.291

Féart, C., Samieri, C., Rondeau, V., Amieva, H., Portet, F., Dartigues, J. F., ... \& BarbergerGateau, P. (2009). Adherence to a Mediterranean diet, cognitive decline, and risk of dementia. Journal of the American Medical Association, 302(6), 638-648. doi: 10.1001/jama.2009.1146

Feldman, G., Hayes, A., Kumar, S., Greeson, J., \& Laurenceau, J.P. (2007). Mindfulness and emotion regulation: The development and initial validation of the Cognitive and Affective Mindfulness Scale-Revised (CAMS-R). Journal of Psychopathology and Behavioral Assessment, 29, 177-190. doi: 10.1007/s10862-006-9035-8

Fengler, A. P., \& Goodrich, N. (1979). Wives of elderly disabled men: The hidden patients. The Gerontologist, 19, 175-183. doi: 10.1093/geront/19.2.175

Fincham, F.D., \& Rogge, R. (2013). Positive-Negative Relationship Quality (PNRQ) Scale. 
Measurement Instrument Database for the Social Science. Retrieved from www.midss.ie Fiocco, A. J., \& Mallya, S. (2015). The importance of cultivating mindfulness for cognitive and emotional well-being in late life. Journal of Evidence-Based Complementary \& Alternative Medicine, 20, 35-40. doi: 10.1177/2156587214553940

Flanagan, J. C. (1978). A research approach to improving our quality of life. American Psychologist, 33(2), 138-147.

Folstein, M. F., Folstein, S. E., \& McHugh, P. R. (1975). “Mini-mental state”: a practical method for grading the cognitive state of patients for the clinician. Journal of Psychiatric Research, 12(3), 189-198.

Francesca, C., Ana, L. N., Jérôme, M., \& Frits, T. (2011). OECD health policy studies help wanted? Providing and paying for long-term care: providing and paying for long-term care (Vol. 2011). OECD Publishing.

Franzen, M. D., Paul, D., \& Iverson, G. L. (1996). Reliability of alternate forms of the Trail Making Test. The Clinical Neuropsychologist, 10(2), 125-129.

Frederick, J., \& Fast, J. (1999). Eldercare in Canada: Who does how much? Canadian Social Trends, 53, 26-32.

Gallager-Thompson, D., \& Coon, D. W. (2007). Evidence-based psychological treatments for distress in family caregivers of older adults. Psychology and Aging, 22, 37-51. doi: $10.1037 / 0882-7974.22 .1 .37$

Gallagher-Thompson, D., Shurgot, G. R., Rider, K., Gray, H. L., McKibbin, C. L.,... Thompson, L. W. (2006). Ethnicity, stress, and cortisol function in Hispanic and non-Hispanic white women: A preliminary study of family dementia caregivers and non-caregivers. The American Journal of Geriatric Psychiatry, 14(4), 334-342. 
Gallicchio, L., Siddiqi, N., Langenberg, P., \& Baumgarten, M. (2002). Gender differences in burden and depression among informal caregivers of demented elders in the community. International Journal of Geriatric Psychiatry, 17, 154-163. doi: 10.1002/gps.538

Garland, E. L., Gaylord, S. A., \& Fredrickson, B. L. (2011). Positive reappraisal mediates the stress-reductive effects of mindfulness: An upward spiral process. Mindfulness, 2(1), 5967. doi: $10.1007 / \mathrm{s} 12671-011-0043-8$

Garland, E., Gaylord, S., \& Park, J. (2009). The role of mindfulness in positive reappraisal. Explore: The Journal of Science and Healing, 5(1), 37-44. doi:10.1016/ j.explore.2008.10.001

Garland, E. L., Hanley, A., Farb, N. A., \& Froeliger, B. (2015). State mindfulness during meditation predicts enhanced cognitive reappraisal. Mindfulness, 6(2), 234-242. doi: $10.1007 / \mathrm{s} 12671-013-0250-6$

Garrido, P., de Blas, M., Del Arco, A., Segovia, G., \& Mora, F. (2012). Aging increases basal but not stress-induced levels of corticosterone in the brain of the awake rat. Neurobiology of Aging, 33(2), 375-382.

Germer, C., Siegel, R., \& Fulton, P. (Eds.) (2005). Mindfulness and psychotherapy. New York: Guilford Press.

Gianaros, P. J., Jennings, J. R., Sheu, L. K., Greer, P. J., Kuller, L. H., \& Matthews, K. A. (2007). Prospective reports of chronic life stress predict decreased grey matter volume in the hippocampus. Neuroimage, 35(2), 795-803. doi: 10.1016/j.neuroimage.2006.10.045

Golden, C, J. (1978). Stroop Color and Word Test. Chicago: Stoelting.

Goldin, P. R., \& Gross, J. J. (2010). Effects of mindfulness-based stress reduction (MBSR) on emotion regulation in social anxiety disorder. Emotion,10(1), 83-91. doi: 


\section{$10.1037 / \mathrm{a} 0018441$}

Greene, J., Taylor, S., \& Johnson, W. (1993). Dementia: Physician approaches to caregiver's problems. Southern Medical Journal, 86, 1036-1039.

Grigsby, J., Kaye, K., Baxter, J., Shetterly, S. M., \& Hamman, R. F. (1998). Executive cognitive abilities and functional status among community-dwelling older persons in the San Luis Valley Health and Aging Study. Journal of the American Geriatrics Society, 46(5), 590596. doi: 10.1111/j.1532-5415.1998.tb01075.x

Gross, C. R., Kreitzer, M. J., Reilly-Spong, M., Wall, M., Winbush, N. Y., Patterson, R., ... Cramer-Bornemann, M. (2011). Mindfulness-based stress reduction versus pharmacotherapy for chronic primary insomnia: A randomized controlled clinical trial. EXPLORE: The Journal of Science and Healing, 7, 76-87. doi: 10.1016/j.explore.2010.12.003

Grossman, P., Niemann, L., Schmidt, S., \& Walach, H. (2004). Mindfulness-based stress reduction and health benefits: A meta-analysis. Journal of Psychosomatic Research, 57, 35-43. doi: 10.1016/S0022-3999(03)00573-7

Habermann, B., \& Davis, L. L. (2005). Caring for family with Alzheimer's Disease and Parkinson's Disease. Journal of Gerontological Nursing, 31(6), 49-54.

Haines, J., Spadaro, K.C., Choi, J., Hoffman, L. A., \& Blazeck, A. M. (2014). Reducing stress and anxiety in caregivers of lung transplant patients: Benefits of mindfulness meditation. International Journal of Organ Transplantation Medicine, 5(2), 50-58.

Hamarat, E., Thompson, D., Zabrucky, K.M., Steele, D., Matheny, K.B., \& Aysan, F. (2001). Perceived stress and coping resource availability as predictors of life satisfaction in young, middle-aged, and older adults. Experimental Aging Research, 27(2), 181-196. 


\section{$10.1080 / 036107301750074051$}

Hanks, R. A., Millis, S. R., Ricker, J. H., Giacino, J. T., Nakese-Richardson, R., Frol, A. B., . . Gordon, W. A. (2008). The predictive validity of a brief inpatient neuropsychologic battery for persons with traumatic brain injury. Archives of Physical Medicine and Rehabilitation, 89(5), 950-957. doi:10.1016/j.apmr.2008.01.011

Harbison, J., Kennelly, S.P., \& Kenny, R.A. (2011). Dementia and cerebrovascular disease. In Neurodegenerative Disorders (pp. 75-83). Springer: London.

Hardiman, O., \& Doherty, C. P. (2011). Neurodegenerative disorders: A clinical guide. Springer London.

Health Canada. (2015). Unleashing innovation: Excellent healthcare for Canada [Naylor report]. Retrieved from http://www.hc-sc.gc.ca/hcs-sss/innovation/index-eng.php

Hennessy, J. W., \& Levine, S. (1979). Stress, arousal, and the pituitary-adrenal system: A psychoendocrine hypothesis. In J. M. Sprague \& A. N. Epstein (Eds.), Progress in Psychobiology and Physiological Psychology: Vol. 8. New York, NY: Academic Press

Henry, J.D., \& Crawford, J.R. (2004). A meta-analytic review of verbal fluency performance following focal cortical lesions. Neuropsychology, 18, 2884-295. doi: 10.1037/08944105.18.2.284

Henry, J. D., \& Crawford, J. R. (2005). A meta-analytic review of verbal fluency deficits in depression. Journal of Clinical and Experimental Neuropsychology, 27(1), 78-101. doi: $10.1080 / 138033990513654$

Herman, J. P., \& Cullinan, W. E. (1997). Neurocircuitry of stress: Central control of the hypothalamo-pituitary-adrenocortical axis. Trends in Neurosciences, 20, 78-84. doi: 10.1016/S0166-2236(96)10069-2 
Hermus, G., Stonebridge, C., Theriault, L. \& Bounajm, F. (2012). Home and community care in Canada: An economic footprint. Ottawa ON: The Conference Board of Canada. Retrieved from http://www.conferenceboard.ca/e-library/abstract.aspx?did=4841

Hill, G., Forbes, W., Berthelot, J. M., Lindsay, J., \& McDowell, I. (1996). Dementia among seniors. Health Reports-Statistics Canada, 8, 7-10.

Ho, S. C., Chan, A., Woo, J., Chong, P., \& Sham, A. (2009). Impact of caregiving on health and quality of life: A comparative population-based study of caregivers for elderly persons and non-caregivers. The Journals of Gerontology Series A: Biological Sciences and Medical Sciences, 71, 1-7. doi: 10.1093/gerona/glp034

Hoaglin, D. C., Iglewicz, B., \& Tukey, J. W. (1986). Performance of some resistant rules for outlier labeling. Journal of the American Statistical Association, 81(396), 991-999.

Hollander, M. J., Liu, G., \& Chappell, N. L. (2009). Who cares and how much? The imputed economic contribution to the Canadian healthcare system of middle-aged and older unpaid caregivers providing care to the elderly. Law \& Governance, 12(2), 42-49.

Hooker, K., Bowman, S. R., Coehlo, D. P., Lim, S. R., Kaye, J., Guariglia, R., \& Li, F. (2002). Behavioral change in persons with dementia: Relationships with mental and physical health of caregivers. The Journals of Gerontology Series B: Psychological Sciences and Social Sciences, 57, P453-P460. doi: 10.1093/geronb/57.5.P453

Hoppes, S., Bryce, H., \& Finlay, E. (2012). The effects of brief mindfulness training on caregivers' well-being. Activities, Adaptation \& Aging, 36, 147-166. doi: $10.1080 / 01924788.2012 .673154$

Hou, R. J., Wong, S. S., Yip, B. K., Hung, A. T., Lo, H. M., Chan, P. H., ... \& Ma, S. H. (2014). The effects of mindfulness-based stress reduction program on the mental health of family 
caregivers: A randomized controlled trial. Psychotherapy and Psychosomatics, 83, 4553. doi: $10.1159 / 000353278$

Jain, S., Shapiro, S. L., Swanick, S., Roesch, S. C., Mills, P. J., Bell, I., \& Schwartz, G. E. R. (2007). A randomized controlled trial of mindfulness meditation versus relaxation training: Effects on distress, positive states of mind, rumination, and distraction. Annals of Behavioral Medicine, 33, 11-21. doi: 10.1207/s15324796abm3301_2

Jeckel, C. M., Lopes, R. P., Berleze, M. C., Luz, C., Feix, L., Argimon, I. I., . . Bauer, M. E. (2010). Neuroendocrine and immunological correlates of chronic stress in 'strictly healthy' populations. Neuroimmunomodulation, 17(1), 9-18.

Jenike, M. A., \& Albert, M. S. (1984). The dexamethasone suppression test in patients with presenile and senile dementia of the Alzheimer's type. Journal of the American Geriatrics Society, 32(6), 441-444.

Jha, A. P., Krompinger, J., \& Baime, M. J. (2007). Mindfulness training modifies subsystems of attention. Cognitive, Affective, \& Behavioral Neuroscience, 7, 109-119. doi: 10.3758/CABN.7.2.109

Jha, A. P., Stanley, E. A., Kiyonaga, A., Wong, L., \& Gelfand, L. (2010). Examining the protective effects of mindfulness training on working memory capacity and affective experience. Emotion, 10, 54-64. doi: 10.1037/a0018438

Ji, J., Zoller, B., Sundquist, K., \& Sundquist, J. (2012). Increased risks of coronary heart disease and stroke among spousal caregivers of cancer patients. Circulation, 125, 1742-1747. doi: 10.1161/CIRCULATIONAHA.111.057018

Johansson, B., Bjuhr, H., \& Rönnbäck, L. (2012). Mindfulness-based stress reduction (MBSR) improves long-term mental fatigue after stroke or traumatic brain injury. Brain Injury, 
26, 1621-1628. doi: 10.3109/02699052.2012.700082

Jolles, J. (1986). Cognitive, emotional and behavioral dysfunctions in aging and dementia. Progress in Brain Research, 70, 15-39.

Kabat-Zinn, J. (1982). An outpatient program in behavioral medicine for chronic pain patients based on the practice of mindfulness meditation: Theoretical considerations and preliminary results. General Hospital Psychiatry, 4, 33-47.

Kabat-Zinn, J. (1990). Full catastrophe living: Using the wisdom of your body and mind to face stress, pain and illness. New York, NY: Random House LLC

Kabat-Zinn, J. (2003). Mindfulness-based interventions in context: Past, present, and future. Clinical Psychology: Science and Practice, 10, 144-156. doi: 10.1093/clipsy.bpg016 Kabat-Zinn, J., Massion, A. O., Kristeller, J., Peterson, L. G., Fletcher, K. E., Pbert, L., ... \& Santorelli, S. F. (1992). Effectiveness of a meditation-based stress reduction program in the treatment of anxiety disorders. American Journal of Psychiatry, 149(7), 936-943.

Karlamangla, A. S., Singer, B. H., McEwen, B. S., Rowe, J. W., \& Seeman, T. E. (2002). Allostatic load as a predictor of functional decline: MacArthur studies of successful aging. Journal of Clinical Epidemiology, 55, 696-710. doi: 10.1016/S08954356(02)00399-2

Keating, N., Fast, J., Frederick, M., Cranswick, K., \& Perrier, C. (1999). Eldercare in Canada: Context, content and consequences. Ottawa: Statistics Canada.

Kelley, K., \& Abraham, C. (2004). RCT of a theory-based intervention promoting healthy eating and physical activity amongst out-patients older than 65 years. Social Science \& Medicine, 59, 787-797. doi: 10.1016/j.socscimed.2003.11.036

Kibby, M. Y., Schmitter-Edgecombe, M., \& Long, C. J. (1998). Ecological validity of 
neuropsychological tests: focus on the California Verbal Learning Test and the Wisconsin Card Sorting Test. Archives of Clinical Neuropsychology, 13(6), 523-534. doi: 10.1016/S0887-6177(97)00038-3

Knight, R. W., Bean, J., Wilton, A.S., \& Lin, E. (2015). Cost-effectiveness of the mindfulnessbased stress reduction methodology. Mindfulness, 6, 1379-1386. doi: 10.1007/s12671015-0404-5

Kögler, M., Brandstätter, M., Borasio, G. D., Fensterer, V., Küchenhoff, J., \& Fegg, M. J. (2015). Mindfulness in informal caregivers of palliative patients. Palliative and Supportive Care, 13, 11-18. doi: 10.1017/S1478951513000400

Lavretsky, H., Epel, E. S., Siddarth, P., Nazarian, N., Cyr, N. S., Khalsa, D. S., ... \& Irwin, M. R. (2013). A pilot study of yogic meditation for family dementia caregivers with depressive symptoms: Effects on mental health, cognition, and telomerase activity. International Journal of Geriatric Psychiatry, 28, 57-65. doi: 10.1002/gps.3790

Lee, B. K., Glass, T. A., McAtee, M. J., Wand, G. S., Bandeen-Roche, K., Bolla, K. I., \& Schwartz, B. S. (2007). Associations of salivary cortisol with cognitive function in the Baltimore memory study. Archives of General Psychiatry, 64, 810-818. doi: 10.1001/archpsyc.64.7.810

Lee, G., Dwyer, J. W., \& Coward, R. T. (1993). Gender differences in parent care: Demographic factors and same-gender preferences. Journal of Gerontology, 48, S9-S16. doi: 10.1093/geronj/48.1.S9

Lee, S., Kawachi, I., \& Grodstein, F. (2004). Does caregiving stress affect cognitive function in older women? The Journal of Nervous and Mental Disease, 192, 51-7. doi: 10.1097/01.nmd.0000106000.02232.30 
Lengacher, C. A., Kip, K. E., Barta, M., Post-White, J., Jacobsen, P. B., Groer, M., ... Shelton, M. M. (2012). A pilot study evaluating the effect of mindfulness-based stress reduction on psychological status, physical status, salivary cortisol, and interleukin-6 among advanced-stage cancer patients and their caregivers. Journal of Holistic Nursing, 30(3), 170-185. doi:10.1177/0898010111435949

Lenze, E. J., Hickman, S., Hershey, T., Wendleton, L., Ly, K., Dixon, D., ... Wetherell, J. L. (2014). Mindfulness-Based Stress Reduction for older adults with worry symptoms and co-occurring cognitive dysfunction. International Journal of Geriatric Psychiatry, 29(10), 991-1000. doi: 10.1002/gps.4086

Lewinsohn, P. M., Seeley, J. R., Roberts, R. E., \& Allen, N. B. (1997). Center for Epidemiologic Studies Depression Scale (CES-D) as a screening instrument for depression among community-residing older adults. Psychology and Aging, 12(2), 277.

Lewis, R. S., Nikolova, A., Chang, D. J., \& Weekes, N. Y. (2008). Examination stress and components of working memory. Stress, 11(2), 108-114. doi: 10.1080/ 10253890701535160

Lezack, M.D. (1995). Neuropsychological assessment. Oxford, U.K.: University Press Inc. Lichtenberger, E. O., \& Kaufman, A. S. (2013). Essentials of WAIS-IV assessment, $2^{\text {nd }}$ ed. New York, NY: Wiley.

Lie, C. H., Specht, K., Marshall, J. C., \& Fink, G. R. (2006). Using fMRI to decompose the neural processes underlying the Wisconsin Card Sorting Test. Neuroimage, 30(3), 10381049. doi: 10.1016/j.neuroimage.2005.10.031

Livingston, G., Manela, M., \& Katona, C. (1996). Depression and other psychiatric morbidity in carers of elderly people living at home. British Medical Journal, 312, 153-156. doi: 
10.1136/bmj.312.7024.153

Lundh, L. (2005). The role of acceptance and mindfulness in the treatment of insomnia. Journal of Cognitive Psychotherapy: An International Quarterly, 19(1), 29-39.

Lupien, S. J., de Leon, M., de Santi, S., Convit, A., Tarshish, C., ... \& Meaney, M. J. (1998). Cortisol levels during human aging predict hippocampal atrophy and memory deficits. Nature Neuroscience, 1, 69-73. doi: 10.1038/271

Lupien, S., Lecours, A. R., Lussier, I., Schwartz, G., Nair, N. P. V., \& Meaney, M. J. (1994). Basal cortisol levels and cognitive deficits in human aging. Journal of Neuroscience, 14(5I), 2893-2903.

Lupien, S. J., Maheu, F., Tu, M., Fiocco, A., \& Schramek, T. E. (2007). The effects of stress and stress hormones on human cognition: Implications for the field of brain and cognition. Brain and Cognition, 65(3), 209-237. doi: 10.1016/j.bandc.2007.02.007

Lykins, E. L. B., Baer, R. A., \& Gottlob, L. R. (2012). Performance-based tests of attention and memory in long-term mindfulness meditators and demographically matched nonmeditators. Cognitive Therapy and Research, 36, 103-114. doi: 10.1007/s10608-0109318-y

Lyons, D. M., Lopez, J. M., Yang, C., \& Schatzberg, A. F. (2000). Stress-level cortisol treatment impairs inhibitory control of behavior in monkeys. Journal of Neuroscience, 20, 7816-7821.

Mackenzie, C. S., Smith, M. C., Hasher, L., Leach, L., \& Behl, P. (2007). Cognitive functioning under stress: Evidence from informal caregivers of palliative patients. Journal of Palliative Medicine, 10, 749-758. doi:10.1089/jpm.2006.0171

Mackenzie, C. S., Wiprzycka, U. J., Hasher, L., \& Goldstein, D. (2009). Associations between 
psychological distress, learning, and memory in spouse caregivers of older adults. Journals of Gerontology Series B: Psychological Sciences and Social Sciences, 64B, 742-746. doi: 10.1093/geronb/gbp076

Mahmoudzadeh, S., Mohammadkhani, P., Dolatshahi, B., \& Moradi, S. (2015). Prediction of psychological well-being based on dispositional mindfulness and cognitive emotion regulation strategies in students. Practice in Clinical Psychology, 3(3), 195-202.

Mahurin, R. K., Velligan, D. I., Hazleton, B., Mark Davis, J., Eckert, S., \& Miller, A. L. (2006). Trail Making Test errors and executive function in schizophrenia and depression. The Clinical Neuropsychologist, 20(2), 271-288. doi: 10.1080/13854040590947498

Mallya, S., \& Fiocco, A. J. (2016). Effects of mindfulness training on cognition and well-being in healthy older adults. Mindfulness, 7(2), 453-465. doi: 10.1007/s12671-015-0468-6

Markesbery, W. R., Kryscio, R. J., Lovell, M. A., \& Morrow, J. D. (2005). Lipid peroxidation is an early event in the brain in amnestic mild cognitive impairment. Annals of Neurology, 58, 730-735. doi: 10.1002/ana.20629

Marshall, G. A., Rentz, D. M., Frey, M. T., Locascio, J. J., Johnson, K. A., \& Sperling, R. A. (2011). Executive function and instrumental activities of daily living in MCI and AD. Alzheimer's \& Dementia: The Journal of the Alzheimer's Association, 7(3), 300-308. http://doi.org/10.1016/j.jalz.2010.04.005

Martin, A., Wiggs, C.L., Lalonde, F., \& Mack, C. (1994). Word retrieval to letter and semantic cues: A double dissociation in normal subjects using interference tasks. Neuropsychologia, 32, 1487-1494. doi: 10.1016/0028-3932(94)90120-1

Mausbach, B. T., Aschbacher, K., Mills, P. J., Roepke, S. K., von Känel, R., Patterson, T. L., ... Grant, I. (2008). A 5-year longitudinal study of the relationships between stress, coping, 
and immune cell beta(2)-adrenergic receptor sensitivity. Psychiatry Research, 160(3), $247-255$.

Mayeux, R. (2003). Epidemiology of neurodegeneration. Annual Review of Neuroscience, 26, 81-104. doi: 10.1146/annurev.neuro.26.043002.094919

McCurry, S. M., Logsdon, R. G., Teri, L., \& Vitiello, M. V. (2007). Sleep disturbances in caregivers of persons with dementia: Contributing factors and treatment implications. Sleep Medicine Reviews, 11, 143-153. doi: 10.1016/j.smrv.2006.09.002

McEwen, B. S. (1998). Protective and damaging effects of stress mediators. New England Journal of Medicine, 338(3), 171-179.

McEwen, B. S. (2008). Central effects of stress hormones in health and disease: Understanding the protective and damaging effects of stress and stress mediators. European Journal of Pharmacology, 583(2), 174-185. doi: 10.1016/j.ejphar.2007.11.071

McEwen, B. S., \& Sapolsky, R. M. (1995). Stress and cognitive function. Current Opinion in Neurobiology, 5, 205-216. doi: 10.1016/0959-4388(95)80028-X

Meerlo, P., Sgoifo, A., \& Suchecki, D. (2008). Restricted and disrupted sleep: Effects on autonomic function, neuroendocrine stress systems and stress responsivity. Sleep Medicine Reviews, 12, 197-210. doi: 10.1016/j.smrv.2007.07.007

Meichenbaum, D. (1996). Stress inoculation training for coping with stressors. The Clinical Psychologist, 49, 4-7.

Mills, P. J., Ziegler, M. G., Patterson, T., Dimsdale, J. E., Hauger, R., Irwin, M., \& Grant, I. (1997). Plasma catecholamine and lymphocyte beta 2-adrenergic receptor alterations in elderly Alzheimer caregivers under stress. Psychosomatic Medicine, 59(3), 251-256.

Minor, H. G., Carlson, L. E., Mackenzie, M. J., Zernicke, K., \& Jones, L. (2006) Evaluation of a 
mindfulness-based stress reduction (MBSR) program for caregivers of children with chronic conditions. Social Work in Health Care, 4, 91-109. doi: 10.1300/J010v43n01_06

Miyake, A., Friedman, N. P., Emerson, M. J., Witzki, A. H., Howerter, A., \& Wager, T. D. (2000). The unity and diversity of executive functions and their contributions to complex "Frontal lobe" tasks: A latent variable analysis. Cognitive Psychology, 41(1), 49-100. doi:10.1006/cogp.1999.0734

Moore, A., \& Malinowski, P. (2009). Meditation, mindfulness and cognitive flexibility. Consciousness and Cognition, 18, 176-186. doi: 10.1016/ j.concog.2008.12.008.

Morris, R. G., Morris, L. W., \& Britton, P. G. (1988). Factors affecting the emotional well-being of the caregivers of dementia sufferers. The British Journal of Psychiatry, 153, 147-156. doi: 10.1192/bjp.153.2.147

Moss, D., Waugh, M., \& Barnes, R. (2008). A Tool for Life? Mindfulness as self-help or safe uncertainty. International Journal of Qualitative Studies on Health and Well-Being, 3, 132-142. doi: 10.1080/17482620801939592

Moynihan, J. A., Chapman, B. P., Klorman, R., Krasner, M. S., Duberstein, P. R., Brown, K. W., \& Talbot, N. L. (2013). Mindfulness-based stress reduction for older adults: Effects on executive function, frontal alpha asymmetry and immune function. Neuropsychobiology, 68(1), 34-43. doi: 10.1159/000350949

Newberg, A. B., Wintering, N., Khalsa, D. S., Roggenkamp, H., \& Waldman, M. R. (2010). Meditation effects on cognitive function and cerebral blood flow in subjects with memory loss: a preliminary study. Journal of Alzheimer's Disease, 20(2), 517-526. doi: 10.3233/JAD-2010-1391 
Norton, M. C., Smith, K. R., Østbye, T., Tschanz, J. T., Corcoran, C., Schwartz, S., ... WelshBohmer, K. A. (2010). Greater risk of dementia when spouse has dementia? The Cache County Study. Journal of the American Geriatrics Society, 58, 895-900. doi:

$10.1111 /$ j.1532-5415.2010.02806.x

O’Bryant, S. E., Humphreys, J. D., Smith, G. E., Ivnik, R. J., Graff-Radford, N. R., Petersen, R. C., \& Lucas, J. A. (2008). Detecting dementia with the Mini-Mental State Examination (MMSE) in highly educated individuals. Archives of Neurology, 65, 963-967. doi:

10.1001/archneur.65.7.963

Öhman, L., Nordin, S., Bergdahl, J., Slunga Birdgander, L., \& Stigsdotter Neely, A. (2007). Cognitive function in outpatients with perceived chronic stress. Scandinavian Journal of Work, Environment, \& Health, 33(3), 223-232.

Oken, B. S., Fonareva, I., Haas, M., Wahbeh, H., Lane, J. B., Zajdel, D., \& Amen, A. (2010). Pilot controlled trial of mindfulness meditation and education for dementia caregivers. Journal of Alternative and Complementary Medicine, 16, 1031-1038. doi: 10.1089/acm.2009.0733

Olszewska, D. A., Fahn, S., Walsh, R.A., \& Lynch, T. (2011). Parkinson’s Disease. In Neurodegenerative Disorders (pp. 85-115). Springer: London.

Ory, M. G., Hoffman, R. R., Yee, J. L., Tennstedt, S., \& Schulz, R. (1999). Prevalence and impact of caregiving: A detailed comparison between dementia and nondementia caregivers. The Gerontologist, 39, 177-186. doi: 10.1093/geront/39.2.177

Ostwald, S. K., Hepburn, K. W., Caron, W., Burns, T., \& Mantell, R. (1999). Reducing Caregiver Burden: A Randomized Psychoeducational Intervention for Caregivers of Persons With Dementia. The Gerontologist, 39 (3 ), 299-309. doi: 


\subsection{3/geront/39.3.299}

Parkinson Society Canada (2003). Parkinson's Disease: Social and Economic Impact. Accessed from: http:/www.parkinson.ca/atf/cf/\%7B9ebd08a9-7886-4b2d-a1c4-

\section{a131e7096bf8\%7D/PARKINSONSDISEASE_EN.PDF}

Parkinson's UK (2017). Dementia. Retrieved from https://www.parkinsons.org.uk/informationand-support/dementia

Parks, S. M., \& Novielli, K. D. (2000). A practical guide to caring for caregivers. American Family Physician, 62(12), 2613-2622.

Perret, E. (1974). The left frontal lobe of man and the suppression of habitual responses in verbal categorical behaviour. Neuropsychologia, 12(3), 323-330.

Perrine, K., Hermann, B. P., Meador, K. J., Vickrey, B. G., Cramer, J. A., Hays, R. D., \& Devinsky, O. (1995). The relationship of neuropsychological functioning to quality of life in epilepsy. Archives of Neurology, 52(10), 997-1003. doi:

10.1001/archneur.1995.00540340089017

Picard, A. (2011, March 25). Canada's dementia dilemma: Care without a strategy. The Globe and Mail. Retrieved from http://www.theglobeandmail.com/life/health-andfitness/health/conditions/canadas-dementia-dilemma-care-without-astrategy/article574243/

Pihlajamaki, M., Tanila, H., Hanninen, T., Kononen, M., Laakso, M. P., Partanen, K., ... \& Aronen, H. J. (2000). Verbal fluency activates the left medial temporal lobe: a functional magnetic resonance imaging study. Neurobiology of Aging, 21, 106. doi: 10.1016/S01974580(00)82275-7

Pinquart, M., \& Sörensen, S. (2003). Differences between caregivers and non-caregivers in 
psychological health and physical health: A meta-analysis. Psychology and Aging, 18, 250-267. doi: $10.1037 / 0882-7974.18 .2 .250$

Pinquart, M., \& Sörensen, S. (2006). Helping caregivers of persons with dementia: Which interventions work and how large are their effects? International Psychogeriatrics, 18, 577-595. doi: 10.1017/S1041610206003462

Porcelli, A. J., \& Delgado, M. R. (2009). Acute stress modulates risk taking in financial decision making. Psychological Science, 20(3), 278-283. doi: 10.1111/j.1467-9280.2009.02288.x

Praissman, S. (2008). Mindfulness-based stress reduction: A literature review and clinician's guide. Journal of the American Academy of Nurse Practitioners, 20(4), 212. doi:10.1111/j.1745-7599.2008.00306.x

Pruchno, R. A., \& Potashnik, S. L. (1989). Caregiving spouses physical and mental health in perspective. Journal of the American Geriatrics Society, 37, 697-705. doi: 10.1111/j.1532-5415.1989.tb02230.x

Pruessner, J. C., Baldwin, M. W., Dedovic, K., Renwick, R., Mahani, N. K., Lord, C., ... \& Lupien, S. (2005). Self-esteem, locus of control, hippocampal volume, and cortisol regulation in young and old adulthood. Neuroimage, 28(4), 815-826. doi: 10.1016/j.neuroimage.2005.06.014

Public Health Agency of Canada. (2014). Mapping connections: An understanding of neurological conditions in Canada. Ottawa: Public Health Agency of Canada.

Radloff, L. S. (1977). The CES-D scale: A self-report depression scale for research in the general population. Journal of Applied Psychological Measurement, 1, 385-401.

Radley, J. J., \& Morrison, J. H. (2005). Repeated stress and structural plasticity in the brain. Ageing Research Reviews, 4(2), 271-287. doi: 10.1016/j.arr.2005.03.004 
Rankin, E. D., Haut, M. W., Keefover, R. W., \& Franzen, M. D. (1994). The establishment of clinical cutoffs in measuring caregiver burden in dementia. The Gerontologist, 34, 828832. doi: $10.1093 /$ geront/34.6.828

Rapgay, L., \& Bystrisky, A. (2009). Classical mindfulness. Annals of the New York Academy of Sciences, 1172, 148-162. doi: 10.1111/j.1749-6632.2009.04405.x

Rasmusson, X. D., Zonderman, A. B., Kawas, C., \& Resnick, S. M. (1998). Effects of age and dementia on the Trail Making Test. The Clinical Neuropsychologist, 12(2), 169-178.

Reitan, R., \& Wolfson, D. (1993). The Halstead-Reitan neuropsychological test battery: Theory and clinical interpretation. Tucson, AZ: Neuropsychology Press.

Reynolds, E. (2006). Vitamin B12, folic acid, and the nervous system. The Lancet Neurology, 5(11), 949-960. doi: 10.1016/S1474-4422(06)70598-1

Radley, J. J., \& Morrison, J. H. (2005). Repeated stress and structural plasticity in the brain. Ageing Research Reviews, 4(2), 271-287. doi: 10.1016/j.arr.2005.03.004

Rezende, T. C. B., Coimbra, A. M. V., Costallat, L. T. L., \& Coimbra, I. B. (2010). Factors of high impacts on the life of caregivers of disabled elderly. Archives of Gerontology and Geriatrics, 51(1), 76-80. doi: 10.1016/ j.archger.2009.08.003

Richmond, L. L., Morrison, A. B., Chein, J. M., \& Olson, I. R. (2011). Working memory training and transfer in older adults. Psychology and Aging, 26(4), 813-822. doi: $10.1037 / \mathrm{a} 0023631$

Rosenberg, M. (1965). Society and the adolescent self-image. Princeton, NJ: Princeton University Press.

Rosser, A., \& Hodges, J. R. (1994). Initial letter and semantic category fluency in Alzheimer's 
disease, Huntington's disease, and progressive supranuclear palsy. Journal of Neurology Neurosurgery and Psychiatry, 57, 1389-1394

Rothman, K. J. (1990). No adjustments are needed for multiple comparisons. Epidemiology, l, $43-46$.

Rhyu, I. J., Bytheway, J. A., Kohler, S. J., Lange, H., Lee, K. J., Boklewski, J., ... \& Cameron, J. L. (2010). Effects of aerobic exercise training on cognitive function and cortical vascularity in monkeys. Neuroscience, 167(4), 1239-1248. doi:

10.1016/j.neuroscience.2010.03.003

Saczynski, J. S., Beiser, A., Seshadri, S., Auerbach, S., Wolf, P. A., \& Au, R. (2010).

Depressive symptoms and risk of dementia: The Framingham Heart

Study. Neurology, 75(1), 35-41. doi: 10.1212/WNL.0b013e3181e62138

Sapolsky, R. M. (1999). Glucocorticoids, stress, and their adverse neurological effects:

relevance to aging. Experimental Gerontology, 34, 721-732. doi: 10.1016/S0531$5565(99) 00047-9$

Schmall, V. L., Cleland, M., \& Sturdevant, M. (2000). The Caregiver Helpbook: Powerful Tools for Caregiving. Legacy Health Systems; Portland, OR.

Schofield, H., Murphy, B., Herrman, H. E., Bloch, S., \& Singh, B. S. (1998). Carers of people aged over 50 with physical impairment, memory loss and dementia: A comparative study. Ageing and Society, 18(03), 355-369.

Schultz, C. L., Smyrnios, K. X., Schultz, N. C., \& Grbich, C. F. (1994). Longitudinal outcomes of psychoeducational support for family caregivers of elderly dependent persons. Australian Psychologist, 28(1), 21-24.

Schulz, R., \& Beach, S. R. (1999). Caregiving as a risk factor for mortality: The Caregiver 
Health Effects Study. Journal of the American Medical Association, 282(23), 2215-2219.

Schulz, R., \& Martire, L. M. (2004). Family caregiving of persons with dementia: Prevalence, health effects, and support strategies. The American Journal of Geriatric Psychiatry, 12, 240-249. doi: 10.1097/00019442-200405000-00002

Schulz, R., Mendelsohn, A. B., Haley, W. E., Mahoney, D., Allen, R. S., Zhang, S., ... \& Belle, S. H. (2003). End-of-life care and the effects of bereavement on family caregivers of persons with dementia. New England Journal of Medicine, 349(20), 1936-1942.

Schulz, R., O’Brien, A. T., Bookwala, J., \& Fleissner, K. (1995). Psychiatric and physical morbidity effects of dementia caregiving: prevalence, correlates, and causes. The Gerontologist, 35, 771-791. doi: 10.1093/geront/35.6.771

Schwiebert, V., \& Myers, J. E. (1994). Midlife caregivers: Effectiveness of a psychoeducational intervention for midlife adults with parent-care responsibilities. Journal of Counseling and Development, 72, 627-632.

Seeman, T. E., Lusignolo, T. M., Albert, M., \& Berkman, L. (2001). Social relationships, social support, and patterns of cognitive aging in healthy, high-functioning older adults: MacArthur studies of successful aging. Health Psychology, 20(4), 243-255. doi: $10.1037 / 0278-6133.20 .4 .243$

Segal, Z. J., Williams, M. G., \& Teasdale, J. D. (2002). Mindfulness based cognitive therapy for depression: A new approach to preventing relapses. New York: Guildford Press.

Shanks-McElroy, H. A., \& Strobino, J. (2001). Male caregivers of spouses with Alzheimer's disease: Risk factors and health status. American Journal of Alzheimer's Disease and Other Dementias, 16, 167-175. doi: 10.1177/153331750101600308

Shao, Z., Janse, E., Visser, K., \& Meyer, A. S. (2014). What do verbal fluency tasks measure? 
Predictors of verbal fluency performance in older adults. Frontiers in Psychology, 5, 772. doi: 10.3389/fpsyg.2014.00772

Shapiro, S. L., Schwartz, G. E., \& Bonner, G. (1998). Effects of mindfulness-based stress reduction on medical and premedical students. Journal of Behavioral Medicine, 21(6), 581-599. doi: 10.1023/A:1018700829825

Sinha, M. (2013). Portrait of caregivers, 2012. Spotlight on Canadians: Results from the General Social Survey. Catalogue no. 89-652-X - No. 001.

Skosnik, P. D., Chatterton, R. T., Swisher, T., \& Park, S. (2000). Modulation of attentional inhibition by norepinephrine and cortisol after psychological stress. International Journal of Psychophysiology, 36(1), 59-68. doi: 10.1016/S0167-8760(99)00100-2

Solfrizzi, V., Colacicco, A. M., D’Introno, A., Capurso, C., Torres, F., Rizzo, C., ... \& Panza, F. (2006). Dietary intake of unsaturated fatty acids and age-related cognitive decline: a 8.5year follow-up of the Italian Longitudinal Study on Aging. Neurobiology of Aging, 27(11), 1694-1704. doi: 10.1016/j.neurobiolaging.2005.09.026

Sonntag, W. E., Ramsey, M., \& Carter, C. S. (2005). Growth hormone and insulin-like growth factor-1 (IGF-1) and their influence on cognitive aging. Ageing Research Reviews, 4, 195-212. doi: 10.1016/j.arr.2005.02.001

Sörensen, S., Pinquart, M., \& Duberstein, P. (2002). How effective are interventions with caregivers? An updated meta-analysis. The Gerontologist, 42, 356-372. doi: $10.1093 /$ geront $/ 42.3 .356$

Sorrell, J.M., \& Cangelosi, P.R. (2009). Caregiver burden or caregiver gain? Journal of Psychosocial Nursing, 47(9), 19-22.

Speca, M., Carlson, L. E., Goodey, E., \& Angen, M. (2000). A randomized, waitlist controlled 
clinical trial: The effect of a mindfulness meditation-based stress reduction program on mood and symptoms of stress in cancer outpatients. Psychosomatic Medicine, 62(5), $613-622$.

Stampfer, M. J., Kang, J. H., Chen, J., Cherry, R., \& Grodstein, F. (2005). Effects of moderate alcohol consumption on cognitive function in women. New England Journal of Medicine, 352(3), 245-253. doi: 10.1056/NEJMoa041152

Starcke, K., \& Brand, M. (2012). Decision making under stress: a selective review. Neuroscience \& Biobehavioral Reviews, 36(4), 1228-1248. doi: 10.1016/j.neubiorev.2012.02.003

Starkman, M. N., Gebarski, S. S., Berent, S., \& Schteingart, D. E. (1992). Hippocampal formation volume, memory dysfunction, and cortisol levels in patients with Cushing's syndrome. Biological Psychiatry, 32(9), 756-765.

Statistics Canada. (2010). An aging population. Retrieved from http://www.statcan.gc.ca/pub/11-402-x/2010000/chap/pop/pop02-eng.htm

Steinberg, B. A., Bieliauskas, L. A., Smith, G. E., Ivnik, R. J., \& Malec, J. F. (2005). Mayo's Older Americans normative studies: Age-and IQ-adjusted norms for the auditory verbal learning test and the visual spatial learning test. The Clinical Neuropsychologist, 19(3-4), 464-523. doi: 10.1080/13854040590945193

Stephen, M.A., Ogrocki, P.K., \& Kinney, J.M. (1991). Sources of stress for family caregivers of institutionalized dementia patients. The Journal of Applied Gerontology 10, 328-342.

Stirman, S. W., Shields, N., Deloriea, J., Landy, M. S., Belus, J. M., Maslej, M. M., \& Monson, C. M. (2013). A randomized controlled dismantling trial of post-workshop consultation strategies to increase effectiveness and fidelity to an evidence-based psychotherapy for 
Posttraumatic stress disorder. Implementation Science, 8(82), 1-8. doi: 10.1186/17485908-8-82.

Stonebridge, C., \& Sutherland, G. (2016). Healthy brains at work: Estimating the impact of workplace mental health benefits and programs. Ottawa: The Conference Board of Canada.

Strauss, E., Sherman, E. M., \& Spreen, O. (2006). A compendium of neuropsychological tests: Administration, norms, and commentary. Toronto: Oxford University Press.

Stuss, D. T., Bisschop, S. M., Alexander, M. P., Levine, B., Katz, D., \& Izukawa, D. (2001). The Trail Making Test: A study in focal lesion patients. Psychological Assessment, 13(2), 230-239. doi: 10.1037/1040-3590.13.2.230

Swanwick, G. R., Kirby, M., Bruce, I., Buggy, F., Coen, R. F., Coakley, D., \& Lawlor, B. A. (1998). Hypothalamic-pituitary-adrenal axis dysfunction in Alzheimer's disease: lack of association between longitudinal and cross-sectional findings. American Journal of Psychiatry, 155(2), 286-289.

Tang, B., Harary, E., Kurzman, R., Mould-Quevedo, J. F., Pan, S., Yang, J., \& Qiao, J. (2013). Clinical characterization and the caregiver burden of dementia in China. Value in Health Regional Issues, 2, 118-126. doi: doi: 10.1016/j.vhri.2013.02.010

Thierry, A.M., Javoy, F., Glowinski, J., \& Kety, S. S. (1968). Effects of stress on the metabolism of norepinephrine, dopamine and serotonin in the central nervous system of the rat: Modifications of norepinephrine turnover. Journal of Pharmacology and Experimental Therapeutics, 163(1), 163-171.

Thomas, P., Lalloué, F., Preux, P. M., Hazif-Thomas, C., Pariel, S., Inscale, R., ... \& Clément, J. P. (2006). Dementia patients caregivers quality of life: the PIXEL study. International 
Journal of Geriatric Psychiatry, 21(1), 50-56. doi: 10.1002/gps.1422

Tierney, M. C., Charles, J., Jaglal, S., Snow, W. G., Szalai, J. P., Spizzirri, F., \& Fisher, R. H. (2001). Identification of those at greatest risk of harm among cognitively impaired people who live alone. Aging, Neuropsychology, and Cognition, 8(3), 182-191.

Tombaugh, T. N. (2004). Trail Making Test A and B: Normative data stratified by age and education. Archives of Clinical Neuropsychology, 19(2), 203-214. doi: 10.1016/S08876177(03)00039-8

Tremont, G., Halpert, S., Javorsky, D. J., \& Stern, R. A. (2000). Differential impact of executive dysfunction on verbal list learning and story recall. The Clinical Neuropsychologist, 14(3), 295-302.

United Nations. (2015). World Population Ageing 2015. New York: United Nations.

Uno, H., Tarara, R., Else, J. G., Suleman, M. A., \& Sapolsky, R. M. (1989). Hippocampal damage associated with prolonged and fatal stress in primates. Journal of Neuroscience, 9(5), 1705-1711.

van Hooren, S. A. H, Valentijn, A. M., Bosma, H., Ponds, R. W. H. M, van Boxtel, M. P. J, \& Jolles, J. (2007). Cognitive functioning in healthy older adults aged 64-81: A cohort study into the effects of age, sex, and education. Aging, Neuropsychology, and Cognition, 14(1), 40-54. doi:10.1080/138255890969483

Vedhara, K., Cox, N. K., Wilcock, G. K., Perks, P., Hunt, M., Anderson, S., ... \& Shanks, N. M. (1999). Chronic stress in elderly carers of dementia patients and antibody response to influenza vaccination. Lancet, 353, 627-631. doi: 10.1016/S0140-6736(98)06098-X

Vitaliano, P. P. (2010). An ironic tragedy: are spouses of persons with dementia at higher risk for dementia than spouses of persons without dementia? Journal of the American 
Geriatrics Society, 58, 976-978. doi: 10.1111/j.1532-5415.2010.02843.x

Vitaliano, P. P., Echeverria, D., Yi, J., Phillips, P. E. M., Young, H., \& Siegler, I. C. (2005).

Psychophysiological mediators of caregiver stress and differential cognitive decline. Psychology and Aging, 20, 402-411. doi: 10.1037/0882-7974.20.3.402

Vitaliano, P. P., Scanlan, J. M., Zhang, J., Savage, M. V, Hirsch, I. B., \& Siegler, I. C. (2002). A path model of chronic stress, the metabolic syndrome, and coronary heart disease. Psychosomatic Medicine, 64(3), 418-435.

Vitaliano, P. P., Young, H. M., \& Zhang, J. (2004). Is caregiving a risk factor for illness? Current Directions in Psychological Science, 13, 13-16. doi: 10.1111/j.09637214.2004.01301004.x

Vitaliano, P. P., Zhang, J., \& Scanlan, J. M. (2003). Is caregiving hazardous to one's physical health? A meta-analysis. Psychological Bulletin, 129, 946-972. doi: 10.1037/00332909.129.6.946

Vitaliano, P. P., Zhang, J., Young, H. M., Caswell, L. W., Scanlan, J. M., \& Echeverria, D. (2009). Depressed mood mediates decline in cognitive processing speed in caregivers. Gerontologist, 49, 12-22. doi: 10.1093/geront/gnp004

von Känel, R., Mausbach, B. T., Patterson, T. L., Dimsdale, J. E., Aschbacher, K., Mills, P. J., ... Grant, I. (2008). Increased Framingham Coronary Heart Disease Risk Score in dementia caregivers relative to non-caregiving controls. Gerontology, 54, 131-137. doi: $10.1159 / 000113649$

Waelde, L. C., Thompson, L., \& Gallagher-Thompson, D. (2004). A pilot study of a yoga and meditation intervention for dementia caregiver stress. Journal of Clinical Psychology, 60, 677-687. doi: 10.1002/jclp.10259 
Wang, Q., Wang, D., Li, C., \& Miller, R. B. (2014). Marital satisfaction and depressive symptoms among Chinese older couples. Aging \& Mental Health, 18, 11-18. doi: $10.1080 / 13607863.2013 .805730$

Watanabe, Y., Gould, E., \& McEwen, B. S. (1992). Stress induces atrophy of apical dendrites of hippocampal CA3 pyramidal neurons. Brain Research, 588(2), 341-345.

Watson, R., Modeste, N. N., Catolico, O., \& Crouch, M. (1998). The relationship between caregiver burden and self-care deficits in former rehabilitation patients. Rehabilitation Nursing, 23, 258-262. doi: 10.1002/j.2048-7940.1998.tb01796.x

Wechsler, D. (2008). Wechsler Adult Intelligence Scale-Fourth Edition. San Antonio, TX: Pearson.

West, A., \& Spring, B. (2002). Randomized controlled trials. Retrieved from http://www.ebbp.org/course_outlines/randomized_controlled_trials/

Whitebird, R. R., Kreitzer, M., Lauren Crain, A., Lewis, B. A., Hanson, L. R., \& Enstad, C. J. (2013). Mindfulness-based stress reduction for family caregivers: A randomized controlled trial. Gerontologist, 53, 676-686. doi: 10.1093/geront/gns126

Willis, S. L., Jay, G. M., Diehl, M., \& Marsiske, M. (1992). Longitudinal change and prediction of everyday task competence in the elderly. Research on Aging, 14, 68-91. doi: $10.1177 / 0164027592141004$

Woolley, C. S., Gould, E., \& McEwen, B. S. (1990). Exposure to excess glucocorticoids alters dendritic morphology of adult hippocampal pyramidal neurons. Brain Research, 531(1), $225-231$.

World Health Organization. (2012). World Alzheimer Report 2012. Retrieved from www.alz.co.uk/research/world-report-2012. 
Zarit, S. H., Reever, K. E., \& Bach-Peterson, J. (1980). Relatives of the impaired elderly: Correlates of feelings of burden. The Gerontologist, 20(6), 649-655.

Zeidan, F., Johnson, S. K., Diamond, B. J., David, Z., \& Goolkasian, P. (2010). Mindfulness meditation improves cognition: Evidence of brief mental training. Consciousness and Cognition, 19, 597-605. doi:10.1016/j.concog.2010.03.01 